\title{
Time Variability of Io's Volcanic Activity from Near-IR Adaptive Optics Observations on 100 Nights in 2013-2015
}

\author{
Katherine de Kleer ${ }^{\mathrm{a}}$, Imke de Pater ${ }^{\mathrm{a}, \mathrm{b}, \mathrm{c}}$ \\ ${ }^{a}$ Astronomy Department, University of California, Berkeley, CA 94720 \\ ${ }^{b}$ Delft Institute of Earth Observation and Space Systems, Delft University of Technology, NL-2629 HS Delft, The Netherlands \\ ${ }^{c}$ SRON Netherlands Institute for Space Research 3584 CA Utrecht, The Netherlands
}

Keywords: Io, Volcanism, Infrared Observations, Adaptive Optics, Spectroscopy

Katherine de Kleer

Department of Astronomy

501 Campbell Hall \#3411

University of California at Berkeley

Berkeley, CA 94720

E-mail: kdekleer@berkeley.edu

(C) 2016. This manuscript version is made available under the Elsevier user license http://www.elsevier.com/open-access/userlicense/1.0/ 


\section{Abstract}

Jupiter's moon Io is a dynamic target, exhibiting extreme and time-variable volcanic activity powered by tidal forcing from Jupiter. We have conducted a campaign of high-cadence observations of Io with the goal of characterizing its volcanic activity. Between Aug 2013 and the end of 2015, we imaged Io on 100 nights in the near-infrared with adaptive optics on the Keck and Gemini N telescopes, which resolve emission from individual volcanic hot spots. During our program, we made over 400 detections of 48 distinct hot spots, some of which were detected $30+$ times. We use these observations to derive a timeline of global volcanic activity on Io, which exhibits wide variability from month to month. The timelines of thermal activity at individual volcanic centers have geophysical implications, and will permit future characterization by others. We evaluate hot spot detection limits and give a simple parameterization of the minimum detectable intensity as a function of emission angle, which can be applied to other analyses.

We detected three outburst eruptions in August 2013, but no other outburst-scale events were observed in the subsequent $\sim 90$ observations. Either the cluster of events in August 2013 was a rare occurrence, or there is a mechanism causing large events to occur closely-spaced in time. We also detected large eruptions (though not of outburst scale) within days of one another at Kurdalagon Patera and Sethlaus/Gabija Paterae in 2015. As was also seen in the Galileo dataset, the hot spots we detected can be separated into two categories based on their thermal emission: those that are persistently active for 1 year or more at moderate intensity, and those that are only briefly active, are time-variable, and often reach large intensities. A small number of hot spots in the latter category appear and subside in a matter of days, reaching particularly high intensities; although these are not bright enough to qualify as outbursts, their thermal signatures follow the same pattern, suggesting that a similar mechanism may be responsible for these events though at a smaller scale.

Two eruptions seen at Kurdalagon Patera in January and April 2015 occurred simultaneously with a brightening of the neutral cloud and plasma torus which are sourced from Io's atmosphere. A plume at Kurdalagon Patera, such as was seen by New Horizons in 2007, could have been responsible for the influx of material that caused these brightenings.

\section{Introduction}

Since before the Voyager fly-bys, Io has been recognized as an unusual and dynamic object (Cruikshank and Murphy, 1973). Io's locked eccentric orbit causes high internal heating generated by tidal forcing from Jupiter (Peale, 1979). The high heat flow of $\sim 2.5 \mathrm{~W} / \mathrm{m}^{2}$ (Matson et al., 1981; Veeder et al., 2012), 30 times that of Earth, causes extreme volcanic activity on this rocky body only slightly larger than Earth's moon. Hundreds of active volcanic sites hosting a wide variety of volcanic styles generate thermal signatures that 
are easily detected from the Earth in the near-infrared, and resolved by telescopes equipped with adaptive optics (Marchis et al., 2000). Large eruptions can exhibit rapid variability on a timescale of hours (Blaney et al., 1995; Rathbun et al., 2014), and may change in power output by orders of magnitude over just a few days (de Pater et al., 2014a; de Kleer et al., 2014).

The extreme conditions on Io make it a unique target for gaining insight into numerous processes that operate throughout the Solar System and beyond. Volcanic plumes reaching more than 100 kilometers above Io's surface have been detected by spacecraft (Strom and Schneider, 1981; Geissler et al., 2008), and contribute to the Solar System's only sulfur-based atmosphere (e.g. Lellouch et al., 1990; Spencer et al., 2005), which is lost at a rate of 1 ton/second to a neutral cloud and dynamic plasma torus in the Jovian system (Schneider and Baganel, 2004). Magmas of an ultramafic composition - containing less silica and more heavy metals than the basalts common on Earth today - have been tentatively detected (McEwen et al., 1998; de Kleer et al., 2014). Volcanic activity of this style was widespread on Earth and the other rocky bodies in early Solar System history when planetary interiors were hotter, making Io a potential active analog for studying these processes (Matson et al., 1998).

Obtaining a complete picture of the variability and spatial distribution of Io's volcanic activity requires observations with high temporal and spatial resolution, covering multiple near-infrared wavelengths so that temperatures and power outputs can be calculated. The Galileo mission provided the first and most extensive characterization of Io's volcanic activity over a multi-year period from 1995-2003 (e.g. Lopes-Gautier et al., 2004; Veeder et al., 2012). Since the beginning of this mission, other space- and ground-based observations have provided snapshots of Io's global activity. Occultation photometry provided the first resolved groundbased observations of individual active sites (Spencer et al., 1997), and has been used extensively to capture Io's activity level during time periods surrounding spacecraft encounters (e.g. Rathbun et al., 2010). The advent of adaptive optics on 8-10 $\mathrm{m}$ telescopes has made it possible to observe more and fainter thermal sources. Marchis et al. (2005) characterized activity on Io at all longitudes during 10 nights of observation at the Keck telescope in December 2001; data at multiple near-infrared wavelengths were used to determine the temperatures and power outputs of many of the sites. The New Horizons fly-by of the Jupiter system provided a unique snapshot of Io's volcanism; night-side observations at 1-2.5 $\mu \mathrm{m}$ with the LEISA instrument measured the hot component of many volcanic eruptions (Spencer et al., 2007; Tsang et al., 2014). Thermal emission is difficult to detect at these wavelengths when Io is in sunlight, and such measurements are hence rare from the ground. Simultaneous measurements with MVIC detected plume activity, while the high cadence of the LORRI instrument confirmed that the detected volcanoes did not exhibit variability on a timescale of seconds to minutes, though one site (E. Girru) changed in intensity in a matter of hours to days, and was seen across multiple wavelengths and instruments (Rathbun et al., 2014; Tsang et al., 2014). 
We have obtained a new dataset covering volcanic activity on Io from August 2013 through December 2015 based on near-infrared adaptive optics images taken on 100 nights during this time period. These data represent the highest-cadence coverage of Io's volcanism at high spatial resolution over such a time period to date, allowing for a unique characterization of the variability of activity on timescales from days to years. Our program, introduced in de Kleer et al. (2014), is optimized for maximal time coverage at $3.8 \mu \mathrm{m}$, and the majority of our observations are therefore taken at only one or two wavelengths and constraints on temperature and power are only possible for $\sim 30 \%$ of hot spot detections. Previous ground-based programs and New Horizons have measured global activity at multiple wavelengths, but these observations typically cover only single or sporadic points in time. While the full time baseline of the Galileo observations spans nearly a decade, high resolution observations from dedicated Io fly-bys were rare, and are generally unable to constrain time variability at the scale of days or weeks that we sample. In addition, the consistent spatial resolution of our observations make it easier to separate real variability from observational biases.

We describe the observations and data reduction and calibration procedures in Section 2, followed by our hot spot photometry methods in Section 3. In Section 4 we present a timeline of 3.8- $\mu$ m activity on Io and discuss activity at individual sites of interest. Implications of our results are discussed in Section 5, and our conclusions are summarized in Section 6. Appendix A contains an extensive analysis of the detection limits and intensity and position uncertainties for this type of observation based on simulated data. Details on our observations and hot spot photometry are tabulated in Appendix B.

The spatial distribution of volcanic activity on Io is an important constraint on models of tidal heat dissipation in Io's interior. The spatial distribution of the hot spots detected in this dataset is the subject of a companion paper (de Kleer and de Pater, 2016a).

\section{Observations and Data Processing}

We imaged Io in the near-infrared $(1-5 \mu \mathrm{m})$ on 100 nights between August 2013 and December 2015 with adaptive optics at the Gemini $\mathrm{N}$ and Keck telescopes. The use of adaptive optics provides the spatial resolution to identify and extract the flux densities of individual volcanic hot spots. The details of the instruments and observations are given in Table B.3 and described below, and example data from each instrument are shown in Figure 1. The complete dataset used in this analysis is shown in Figure 2.

\subsection{Keck II NIRC2}

With the Keck II telescope we aim to image all Ionian longitudes every year to track global variability from year to year. The observations presented here are made up three epochs: Aug 2013-Mar 2014 (10 
observations), Oct 2014-Jan 2015 (6 observations), and Mar 2015-Dec 2015 (10 observations). Our observations covered all Ionian longitudes during each of these epochs. However, conditions were poor during multiple nights in the 2013-2014 epoch, and data at some longitudes are unusable. Data from some dates in August 2013 were previously published in de Pater et al. (2014a), and are included here in the context of the full dataset.

We observe Io with the NIRC2 imager combined with the adaptive optics system in natural guide star (NGS) mode, using Io itself for wavefront sensing. The NIRC2 detector is a 1024x1024 Aladdin InSb array, which we use with the narrow camera to obtain a 0.00994"/pixel platescale (de Pater et al., 2006). Io's angular size ranges from 0.8-1.2" depending on its distance from Earth, corresponding to $30-45 \mathrm{~km} /$ pixel at disk center. We image Io in reflected sunlight in a variety of wide- and narrow-band filters between $1.21 \mu \mathrm{m}$ (J-cont) and $4.67 \mu \mathrm{m}(\mathrm{Ms})$. We observe a nearby photometric standard star which we use for flux calibration, for point spread function (PSF) estimation, and to correct for atmospheric absorption. We observe both targets at three locations on the detector and construct a sky frame by median-averaging these frames.

We apply standard data reduction procedures for flat-fielding, bad pixel removal, and sky subtraction, and include a rectification of the images to correct for known spatial distortion using dewarp routines ${ }^{1}$. Target images within a filter are aligned and median-averaged after reduction and sky-subtraction.

\subsection{Gemini N NIRI}

With Gemini N we observed Io briefly over a range of Ionian longitudes on 74 nights between August 2013 and April 2015. We image Io in the K-cont and L' and filters (2.27 and $3.78 \mu \mathrm{m}$, respectively) with the Near-Infrared Imager (NIRI; Hodapp et al., 2003) combined with the ALTAIR adaptive optics system in NGS mode, using Io itself for wavefront sensing. The detector is a 1024x1024 Aladdin InSb array, which we use with the $\mathrm{f} / 32$ camera for a platescale of 0.022 " / pixel, corresponding to $\sim 45$ pixels across the disk of Io, or $\sim 80 \mathrm{~km} /$ pixel at disk center. Our spatial resolution at L', defined as the full width at half maximum of the point spread function, is generally $4-5$ pixels, or $\sim 0.1 "$. We image Io in reflected sunlight at nine detector locations and construct a sky frame by median-averaging the images. We occasionally observe a standard star as well, which we use only for PSF estimation. We apply standard data reduction procedures, including flat-fielding, bad pixel removal, and sky-subtraction. The nine images are then aligned and median-averaged to produce a final image for each night.

Reduced images from both telescopes in a variety of filters are shown in Figure 1.

\footnotetext{
${ }^{1}$ provided by Brian Cameron of the California Institute of Technology
} 

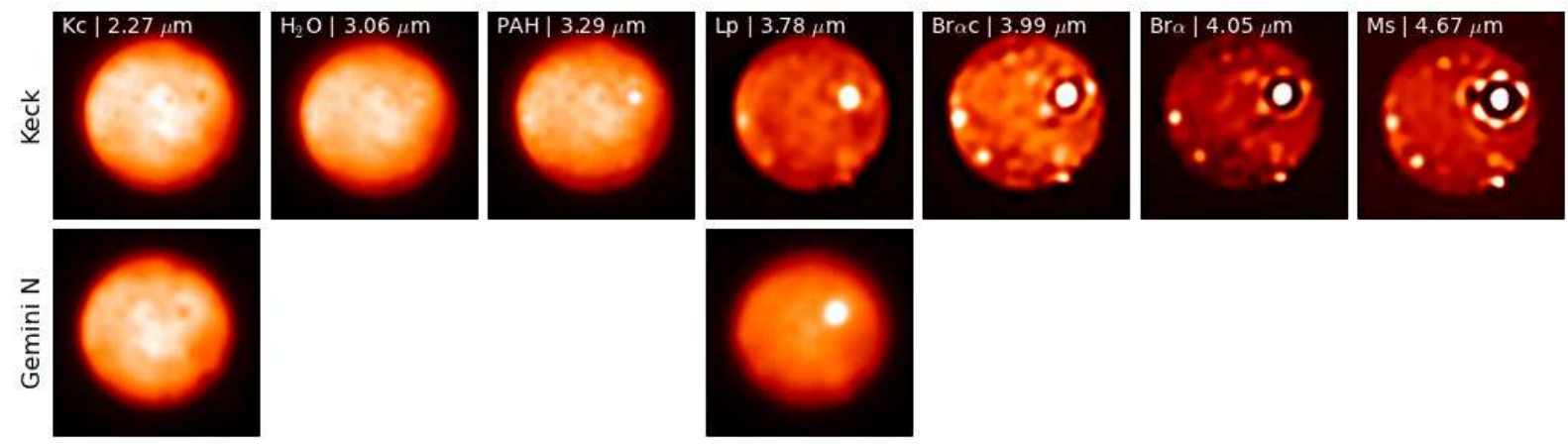

Figure 1: Example observations from the Keck and Gemini N telescopes. The two datasets are from 2015 Jan 12 and 14 for Keck and Gemini N respectively, and are selected for similar activity level and central meridian longitude, to facilitate comparison. Images are labeled with filter name and central wavelength.

\subsection{Flux Calibration}

The Keck observations are flux calibrated using observations of bright photometric standard stars taken on the same night as the target observations. The star counts are extracted from the reduced images via standard aperture photometry, and the Io images are flux calibrated based on the known stellar magnitudes (Leggett et al., 2003; 2006), interpolating between recorded values for narrowband filters and correcting for the airmass difference between the star and Io.

To determine the accuracy of the flux calibration each night, the flux density from reflected sunlight is measured on a region of Io's disk free from volcanic activity. While the emission from volcanically-active regions is highly time-variable, the reflected sunlight from non-active areas is relatively constant in time, after correcting for the sun-Io distance. A reference value for the flux density of Io's disk is determined for each filter based on observations on the most photometric nights. The calibrated background levels for this set of observations only differ by a few percent from one another. If the background level on a given night differs significantly $(>10 \%)$ from this reference value, the entire image is scaled so that the disk background matches the reference value. Of the 26 nights of Keck observations, 19 had good observing conditions and were calibrated to the star; of these, 8 nights were particularly photometric and constituted the referenced set. The remaining 7 nights required correction due to poor weather or lack of a standard star observation.

On most nights, we do not observe a standard star with Gemini N, and we calibrate our Gemini N images using the Keck reference set by the same method described above. The uncertainty in this calibration method is due primarily to Io's non-uniform albedo, as well as background contamination by bright hot spots whose PSF wings may raise the background level of the entire disk. Based on these effects, we derive a a typical uncertainty of $6 \%$ in the photometry resulting from this procedure. This procedure and the derivation of uncertainties is described in further detail in de Kleer et al. (2014), where the program at Gemini $\mathrm{N}$ is introduced. 


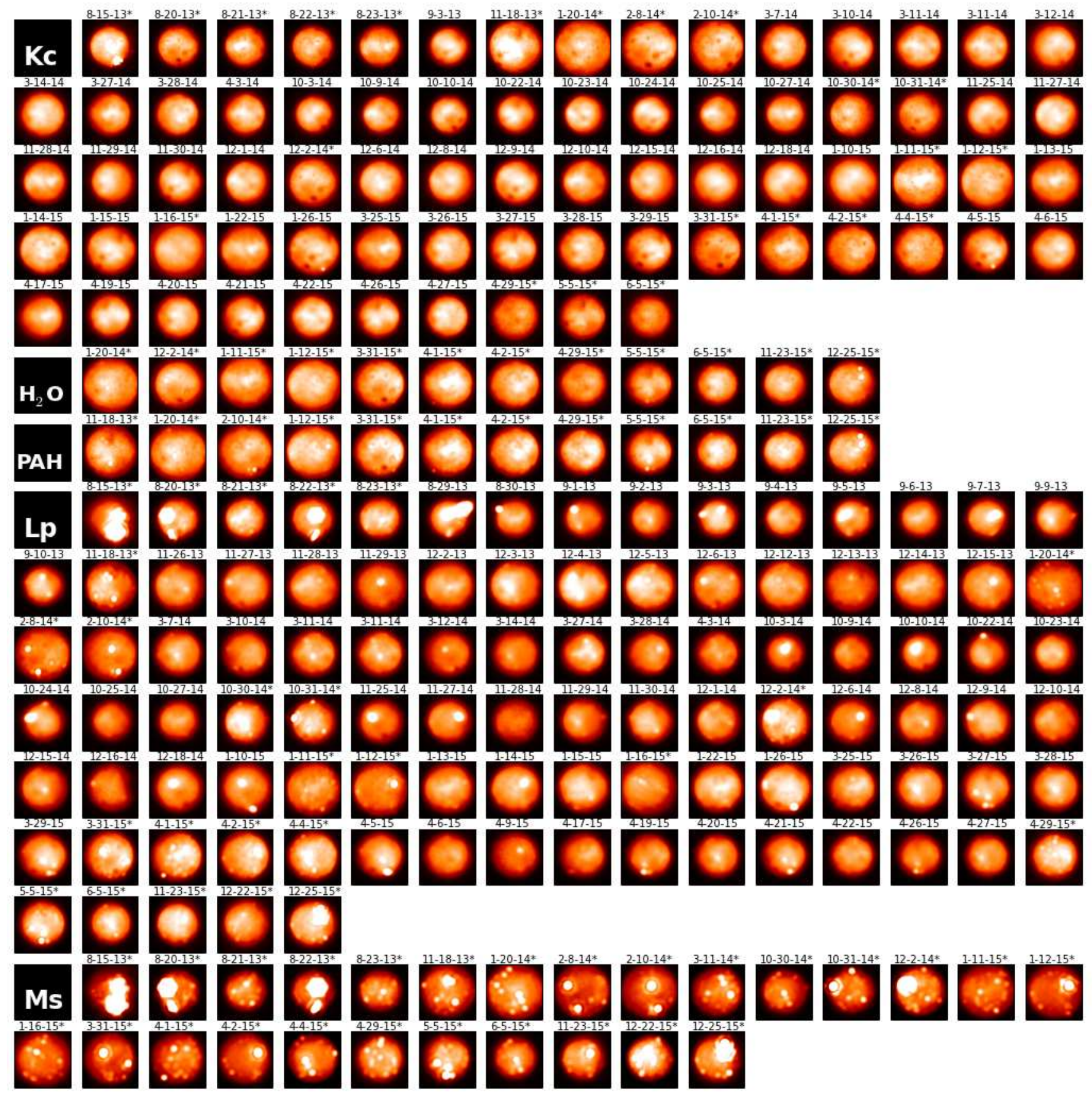

Figure 2: All Io images used in this analysis, taken in five filters: Kc $(2.27 \mu \mathrm{m}), \mathrm{H}_{2} \mathrm{O}(3.06 \mu \mathrm{m}), \mathrm{PAH}(3.36 \mu \mathrm{m}), \mathrm{Lp}(3.78 \mu \mathrm{m})$ and Ms $(4.67 \mu \mathrm{m})$. Dates are indicated above images; asterisks next to dates indicate data from the Keck telescope; all other data are from the Gemini $\mathrm{N}$ telescope.

\subsection{Navigation}

We determine the latitude and longitude of each hot spot detection by navigating the images as follows. 3 To determine the exact center of Io's disk in the images, we fit a circle matching the angular size of Io at the 
date and time of observation ${ }^{2}$ to the limb of the disk by eye. We use the sub-observer latitude and longitude and north polar angle ${ }^{2}$ to convert the $(x, y)$ positions of each spot to Ionian (lat, lon) pairs.

The greatest uncertainty in our latitude-longitude estimates arises from the difficulty in determining the exact location of the limb, particularly at longer wavelengths where the disk is dimmer, and when there is a hot spot near the limb that distorts Io's apparent shape. We estimate that the position we find for Io's center based on the limb fit is accurate to 0.5 pixels for data from both telescopes. This is converted to a latitude and longitude uncertainty by shifting the fitted $(x, y)$ position of each hot spot by half a pixel in each direction and calculating the corresponding (lat,lon) position; the resultant uncertainties therefore reflect the fact that position estimates become less precise closer to the limb. Typical uncertainties derived by this method range from $\sim 0.5^{\circ}$ in Ionian latitude/longitude near disk center to a few degrees near the limb. We evaluate these uncertainty estimates by looking at the actual spread in the positions derived for individual hot spots on multiple images taken on the same night, either in the same filter or different filters. In the Keck images, the spread in positions is typically less than $1.0^{\circ}$ in latitude or longitude, and only rises above $3.0^{\circ}$ on very rare occasions. This corresponds well with the uncertainties we derive. We cannot apply this analysis to our Gemini N observations where only one image is obtained each night, but we might expect the position uncertainties to be $20 \%$ larger than at Keck due to the lower spatial resolution.

In Appendix A we revisit these uncertainties based on an analysis of simulated hot spots inserted into our observations at a range of emission angles and intrinsic flux densities. In some cases, we find that the method above underestimates uncertainties, and we increase errorbars accordingly.

\section{Analysis}

\subsection{Hot Spot Photometry}

We determine the exact position of each hot spot by finding the centroid of the brightest portion of a small sub-image manually chosen to contain only a single hot spot. In cases of faint hot spots near the limb or very near brighter hot spots, the positions found by this method are skewed by nearby brighter pixels, and the positions are corrected manually by eye.

Io is covered by hot spots whose point spread functions overlap, and an aperture that collects all of the flux from one hot spot would be contaminated by others, requiring the use of smaller apertures that do not collect all the flux. The stellar PSF is used to estimate the fraction of flux lost due to this small aperture size and a correction factor is applied to the retrieved hot spot flux density, following the method described by Gibbard et al. (2005; for details, see also: de Pater et al., 2014b). The choice of aperture size generally

\footnotetext{
${ }^{2}$ Ephemeris from JPL HORIZONS System: ssd.jpl.nasa.gov/horizons.cgi
} 
does not impact the extracted flux density beyond the $1 \sigma$ uncertainties, although smaller apertures lead to greater uncertainties. We therefore consider this correction robust except in cases when weather conditions are poor and the PSF varies in shape over the course of the night.

The uncertainties on the correction factor are determined by calculating the spread in values derived for this factor from multiple star observations in the same filter on a given night. This spread is affected by the choice of aperture size, the weather conditions, and the wavelength of observation. At Gemini N we do not observe a standard star each night of observation, and the aperture-size correction factor is based on a set of stellar observations with the same instrument on different nights, which leads to a larger spread and correspondingly higher flux density uncertainties. The factor we apply to the hot spot photometry is the median of the factors derived for each stellar observation for a given aperture choice, and we incorporate the standard deviation of the stellar correction factors into the uncertainty on the hot spot photometry. The stellar observations are made under a variety of weather conditions, and this spread gives us a conservative uncertainty estimate of $\sim 10-20 \%$.

The hot spot intensities are corrected for geometric foreshortening by dividing by $\mu$, the cosine of the emission angle. The final value for the hot spot intensity is calculated as

$$
F_{h s}=F_{h s, \text { counts }} \times \frac{R \times L}{\mu}
$$

where $F_{h s, \text { counts }}$ is the measured hot spot intensity in counts, $R$ is the flux calibration factor converting counts to $\mathrm{GW} / \mu \mathrm{m} / \mathrm{sr}$ based on either stellar or background calibration (see Section 2.3 ), and $L$ is the aperture-size correction factor. The uncertainties are then calculated as

$$
\left(\frac{\sigma_{F, h s}}{F_{h s}}\right)^{2}=\left(\frac{\sigma_{R}}{R}\right)^{2}+\left(\frac{\sigma_{L}}{L}\right)^{2}+\left(\frac{\sigma_{\mu}}{\mu}\right)^{2}
$$

The uncertainty in the flux calibration $\sigma_{R}$, and on the correction factor $\sigma_{L}$ are typically in the $2-10 \%$ range, depending primarily on wavelength and observing conditions. The uncertainty on $\mu$ is calculated from the uncertainty in the position determined for each hot spot. For positions very near the limb, $\sigma_{\mu}$ is much larger in moving towards the limb than away from it, and asymmetric uncertainties are given.

\subsection{Detection Limits and Uncertainties}

A robust characterization of the uncertainties on position and intensity retrievals is crucial for hot spot identification and correct interpretation of time variability. We assess the position and intensity uncertainties calculated by the methods described above by retrieving fake hot spots inserted into observations and testing our retrieval accuracy. This analysis is described in detail in Appendix A. 
The detection limit analysis finds that uncertainties are underestimated for hot spots that are faint and/or near the limb, and the errorbars on these values are increased according to our results from the retrieval accuracy test. Apart from an analysis of the hot spot detection limits of the LEISA instrument on New Horizons (Tsang et al. 2014), a quantitative analysis of instrument sensitivities as they relate to hot spot detections has often been lacking. Such studies are critical for understanding hot spot variability and interpreting non-detections.

\subsection{Temperature and Emitted Power}

On nights when a hot spot is detected at two or more wavelengths, its temperature and emitting area are calculated assuming a single-temperature blackbody model. Although an actual eruption emits at a continuous range of temperatures, the data are typically well fit by a single temperature component, and therefore do not merit a more complex model. The fitted values can thus be considered a rough estimate of the dominant temperature component in the eruption. In cases where observations were made at K-cont but no detection was made, the non-detection is taken as a constraint on the fit.

Models are fit to the data using an affine-invariant ensemble Markov Chain Monte Carlo (MCMC) method, via the Python emcee implementation (Foreman-Mackey et al., 2013). This method is described in detail in de Kleer et al. (2014), where we used it to fit models to the 2-5 $\mu$ m spectrum of an outburst on Io. We perform each run with 40 chains and 1000 steps; the chains typically converge after $<200$ steps. We fit for the temperature and area in log space, and the errorbars are quoted directly from the posterior distributions of these properties. The power $P$ is calculated from the area $A$ and temperature $T$ according to the Stefan-Boltzmann Law: $P=A \sigma T^{4}$, assuming an emissivity of $\epsilon=1$. Figure 3 shows example fits for different cases.
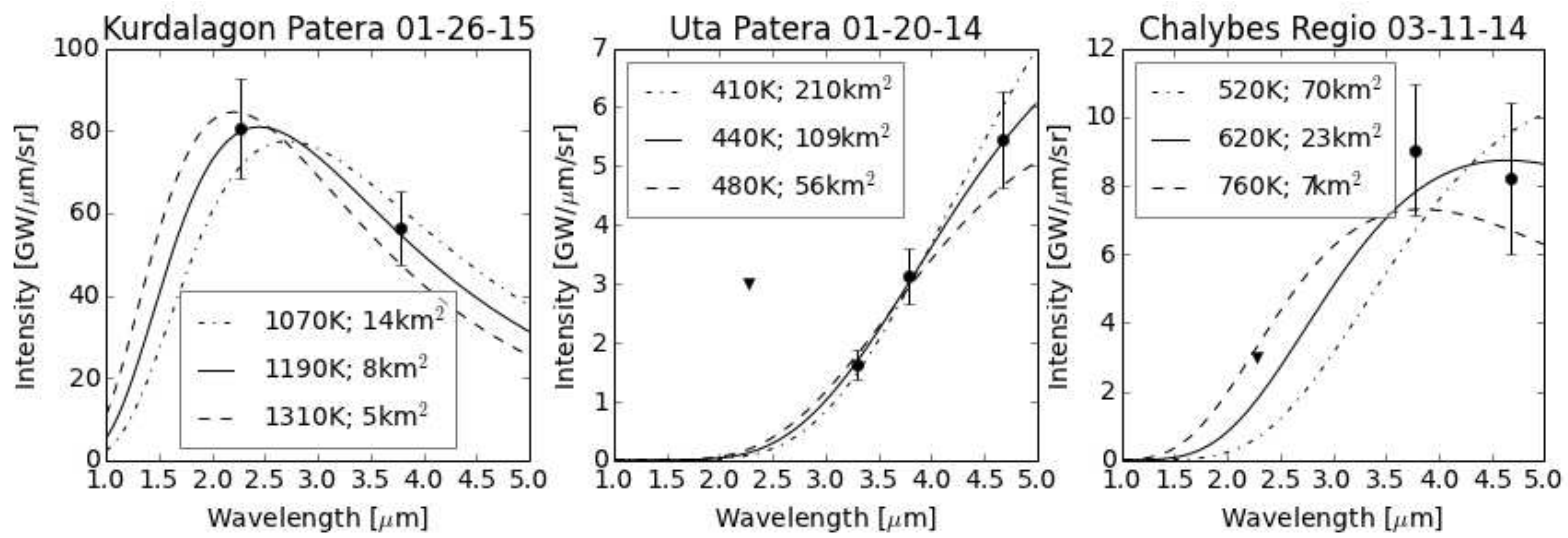

Figure 3: Example blackbody fits to three hot spots: (left) high-temperature hot spot; (middle) low-temperature hot spot; (right) poorly-constrained temperature. The solid curve is the best fit, and the two other curves demonstrate the fits corresponding to the 1- $\sigma$ limits on temperature. Downward arrows are upper limits, and the temperature and area for each fit are indicated. 


\subsection{On L-band Emission as a Tracer of Total Power}

In the absence of multi-wavelength data, the temperature and emitting area cannot be calculated from the observations. However, the majority of our data is taken in at least the Kc and L' filters (wavelengths of 2.3 and $3.8 \mu \mathrm{m}$ ), and a detection at L' combined with a lack of detection at Kc places an upper limit on the temperature that is at or below $\sim 800 \mathrm{~K}$. In addition, the limited range of plausible eruption temperatures allows for a rough estimate of total power from the L'-band intensity alone. This is demonstrated in Figure 4, where spectra at a range of temperatures are shown that all produce the same L'-band intensity. For eruption temperatures above $500 \mathrm{~K}$, the power emitted is within a factor of 3 regardless of the temperature; if we allow eruptions as cool as $400 \mathrm{~K}$, we can only be certain of the power to within a factor of $\sim 8$. A temperature of $400 \mathrm{~K}$, or $127^{\circ} \mathrm{C}$, is at the low end for temperatures we might expect for sulfur volcanism, and too cool for erupting silicate lavas. This is therefore a conservative lower bound on eruption temperature, and we would expect most eruptions to be hotter. Although cooling lava flows may be even colder than this, such flows must cover vast areas to be detectable at $3.8 \mu \mathrm{m}$. In the detections where we have the data to make a temperature measurement, we find the dominant temperature component at the majority of hot spots to be between $500-700 \mathrm{~K}$. This is much lower than the $1475 \mathrm{~K}$ of basaltic magma, and is likely dominated by large areas of cooling lava flows.

This demonstrates that even in the absence of multi-wavelength information, we can constrain the total emitted power to within a factor of $\sim 5$ from the 3.8- $\mu$ m emission alone. Since the variability of the L'-band intensities can be much higher than this, we see that a comparison of L'-band emission between dates and hot spots can provide at least a crude stand-in for a comparison of total power.

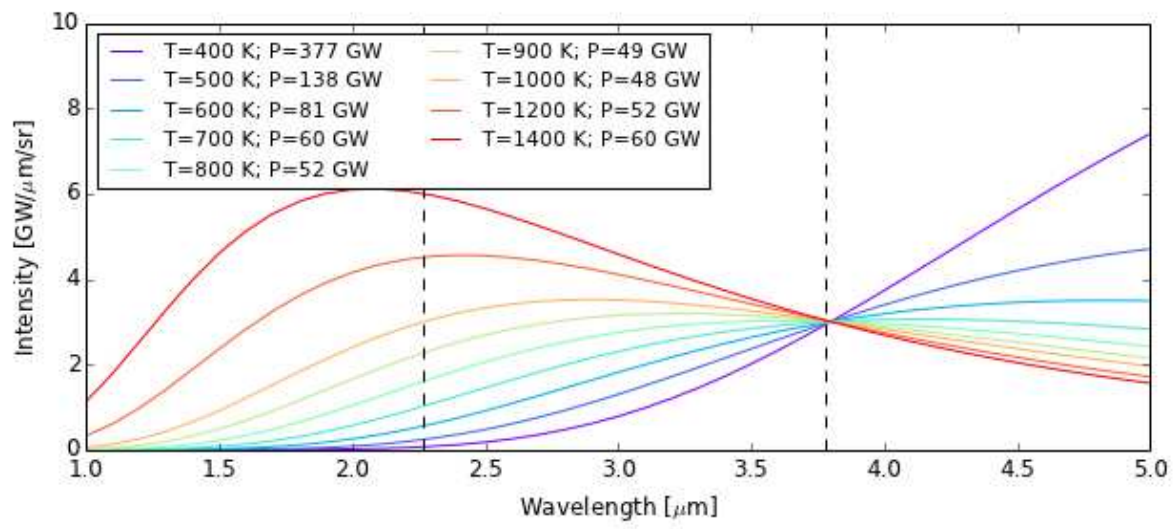

Figure 4: Demonstration of thermal emission spectra at a range of plausible eruption temperatures that produce the same 3.8- $\mu \mathrm{m}$ intensity. Although the spectra differ substantially, the spread in total powers is less than a factor of 10 . The dashed vertical lines correspond to the central wavelengths of the K-cont and L' filters, at 2.27 and $3.78 \mu$ m respectively. 


\section{Results: Time Variability of Volcanic Activity}

Between August 2013 and December 2015, we made 409 detections of thermal activity at 48 distinct hot spots. The timeline of 3.8- $\mu \mathrm{m}$ activity is shown in Figure 5, and all measured hot spot intensities and positions are given in Table B.4, along with identifications of candidate corresponding surface features. This timeline gives a picture of Io's global activity level over timescales of days to months to years, as well as the variability of individual volcanic sites. Of the 48 sites detected, Loki Patera was detected the most frequently (46 times), followed by Janus Patera (32 times) and Marduk Fluctus (29 times); all three sites were detected every time the observing geometry was favorable. Twelve additional sites were detected ten or more times.

Figure 5 includes a timeline of longitudinal coverage during the time period of observation. There were seven $~ 1-$ month intervals of complete longitudinal coverage, and one period of partial coverage. Complete coverage specifically means that any eruption during these dates that sustained a 3.8- $\mu \mathrm{m}$ flux density above $\sim 3 \mathrm{GW} / \mu \mathrm{m} / \mathrm{sr}$ for three weeks or more would have been detected.

The scale of Io's volcanic activity varies widely from month to month, as seen in Figure 5 . In the month of August 2013 four hot spots were detected (Rarog Patera, Heno Patera, 201308C, Loki Patera) with flux densities an order of magnitude brighter than the brightest event detected in the entire period from Nov 2013-Jan 2014, despite excellent longitudinal coverage during much of the latter interval. In general, we detected significant activity in Aug-Sep 2013 followed by a period from Nov 2013-Apr 2014 when we detected only moderate activity (no event brighter than $30 \mathrm{GW} / \mu \mathrm{m} / \mathrm{sr}$ at $3.8 \mu \mathrm{m}$ ). When we began observing again in Oct 2014, Io had multiple regions of high activity, though nothing of an outburst scale; this heightened level of activity continued through the spring of 2015 .

Io's volcanoes exhibit a wide range of time-variability signatures, corresponding to a variety of eruption styles (Davies et al. 2010). While some sites maintain a steady level of low-intensity activity for years, others vary by orders of magnitude, or may appear and subside in just a few weeks or less. In the following sections, we describe activity at individual sites of interest, including the brightest observed activity (Section 4.1), previously-undetected hot spots (Section 4.2); an unusual eruption at Kurdalagon Patera (Section 4.3); and activity at Pele, Amirani, and Loki Patera (Sections 4.4-4.6). The positions stated in bold for each location are an average calculated from our observations based on all hot spot detections attributed to this site (see Table B.4). These locations are shown on a surface map for selected hot spots of interest in Figure 6. Hot spots are identified with the most probable associated surface feature using the feature name when possible. The $\mathrm{P} / \mathrm{PV}$ designations (Radebaugh 2005) are used for unnamed paterae, and patera floor unit ID numbers (Williams et al. 2011a; 2011b) are used when no alternative designation exists.

\footnotetext{
${ }^{4}$ http://astrogeology.usgs.gov/search/map/Io/Voyager-Galileo/Io_GalileoSSI-Voyager_Global_Mosaic_1km
} 
Timeline of 3.8- $\mu \mathrm{m}$ Emission

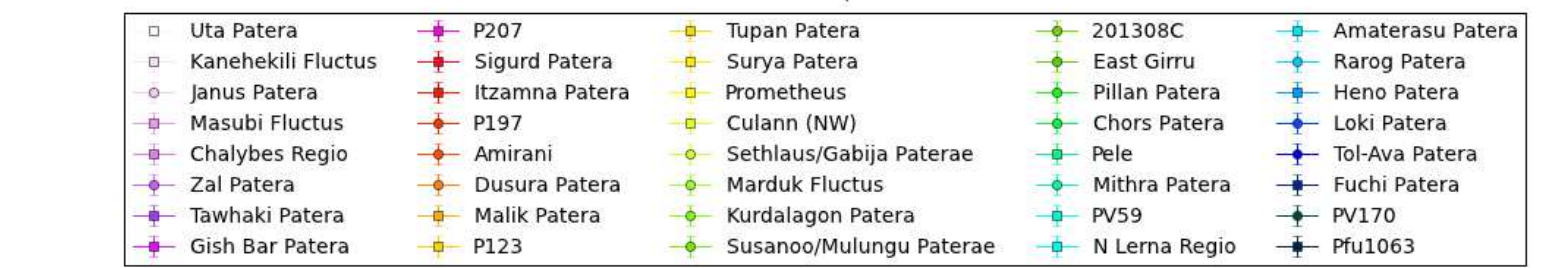
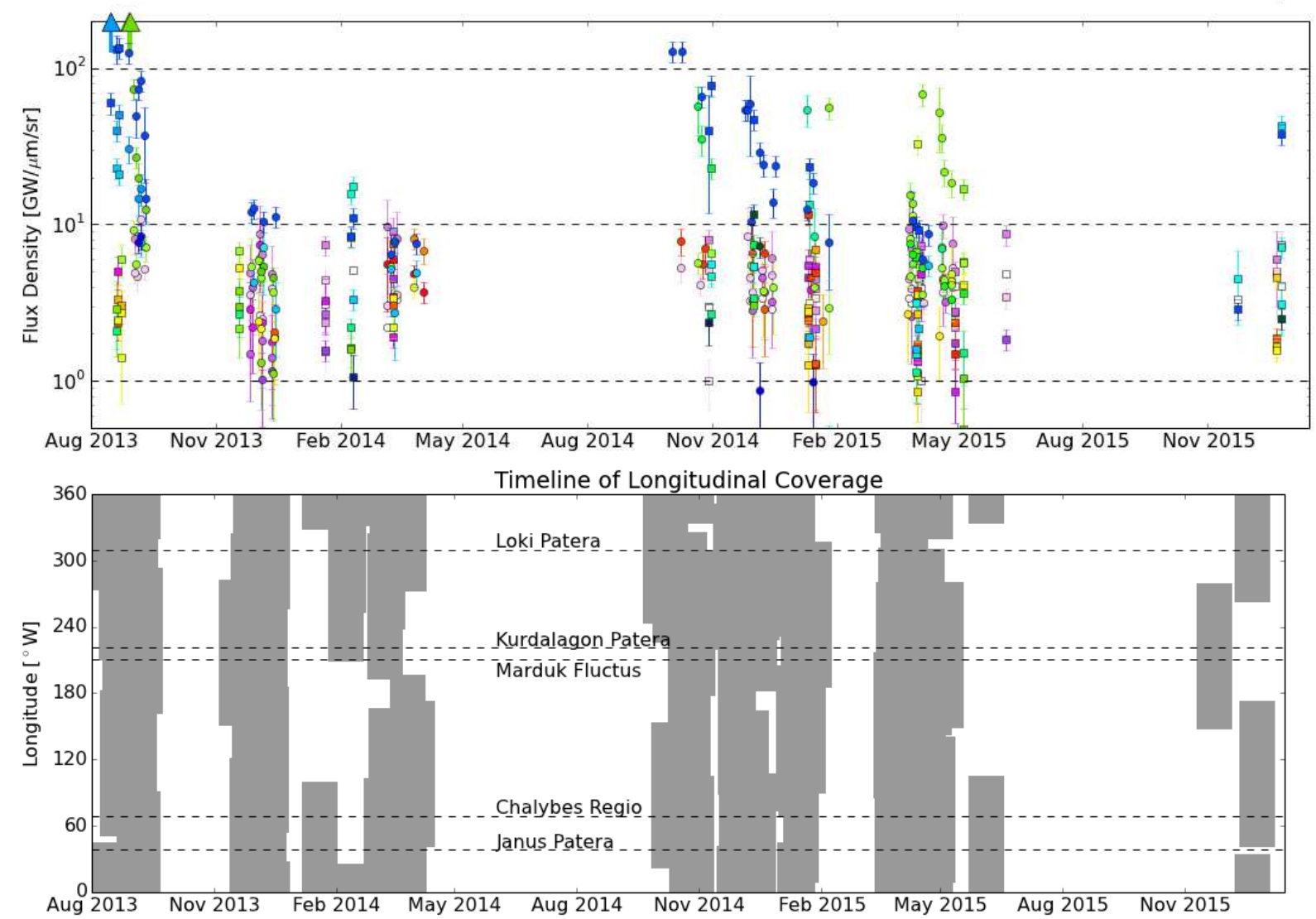

Figure 5: [Top] Timeline of 3.8- $\mu \mathrm{m}$ emission from volcanic activity on Io between August 2013 and December 2015 . Squares are detections with Keck/NIRC2, circles are detections with Gemini N/NIRI, and arrows indicate points off the scale of the figure. [Bottom] Timeline of longitude coverage of observations. The longitudes of selected hot spots discussed in the paper are indicated with dashed lines.

\subsection{Bright Events}

Following the three previously-published outburst events in August 2013 (de Pater et al., 2014a; de Kleer et al., 2014), no other outburst-scale events were detected. A high-energy eruption was detected at Pillan

Patera $\left[\mathbf{1 2}^{\circ} \mathbf{S}, \mathbf{2 4 5}^{\circ} \mathbf{W}\right]$ on Feb $18^{5}$ (de Pater et al., 2016a). We first detected activity at Pillan Patera 5 on March 27, the first date we observed its location after the eruption. During the subsequent months we 6 observed its steady decline in brightness. We detected three brightening events at Loki Patera, which are the subject of a companion paper (de Kleer and de Pater, 2016b). Apart from the outbursts, Loki Patera,

\footnotetext{
${ }^{5}$ Detected at the IRTF by R. Howell.
} 


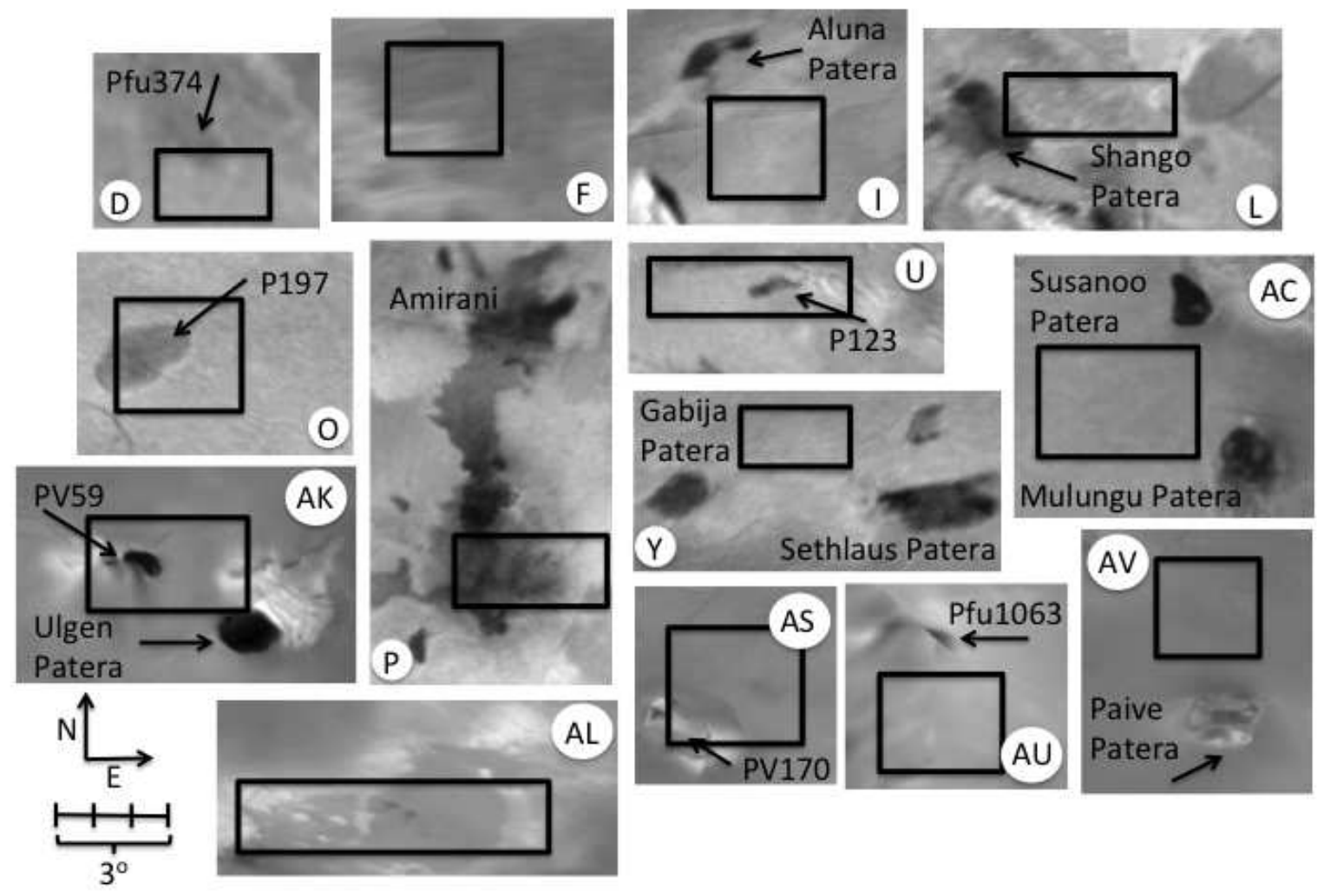

Figure 6: Locations of selected hot spots overlain on the USGS merged Voyager/Galileo SSI Io surface map ${ }^{4}$. Size of rectangles indicate uncertainties on detected location, and letters in bubbles correspond to hot spot identifications in Tables B.4 and 1. In the cases where the rectangles lie between two sources, emission may be present from both but unresolved.

1 and the eruption at Pillan Patera, the brightest activity detected during this period was at Kurdalagon Patera $\left[49^{\circ} \mathbf{S}, 221^{\circ} \mathbf{W}\right](\sim 68 \mathrm{GW} / \mu \mathrm{m} / \mathrm{sr})$, Chors Patera $\left[\mathbf{6 5}^{\circ} \mathbf{N}, \mathbf{2 4 7}^{\circ} \mathbf{W}\right](\sim 57 \mathrm{GW} / \mu \mathrm{m} / \mathrm{sr})$, Mithra Patera $\left[\mathbf{5 8}^{\circ} \mathbf{S}, \mathbf{2 6 6}^{\circ} \mathbf{W}\right](\sim 55 \mathrm{GW} / \mu \mathrm{m} / \mathrm{sr})$, Amaterasu Patera $\left[\mathbf{3 9}^{\circ} \mathbf{N}, \mathbf{3 0 4}^{\circ} \mathbf{W}\right](\sim 43 \mathrm{GW} / \mu \mathrm{m} / \mathrm{sr})$, and Sethlaus/Gabija Paterae $\left[\mathbf{5 0}^{\circ} \mathbf{S}, \mathbf{1 9 8}^{\circ} \mathbf{W}\right](\sim 33 \mathrm{GW} / \mu \mathrm{m} / \mathrm{sr}$; intensities given at $3.8 \mu \mathrm{m})$. In all cases, the peak brightnesses were measured at the first detection of activity from these sites, and were followed by a steady decline in brightness (this cycle repeated twice in the case of Kurdalagon). We note that all sites where bright activity was seen, including these four locations, the three outburst events, Loki Patera, and - Pillan Patera, fall within a $\sim 100^{\circ}$ strip of longitude from $200-300^{\circ} \mathrm{W}$, despite our comparable coverage of 9 the two hemispheres. The significance of this spatial grouping is discussed in de Kleer and de Pater (2016a).

\subsection{New Hot Spots \& Hot Spots without Associated Surface Features}

We detected several hot spots where activity has rarely or never been seen before, some with no clear accompanying patera in Galileo/Voyager surface images. The most distinctive is a hot spot in Chalybes 
Regio $\left[56^{\circ} \mathbf{N}, 68^{\circ} \mathbf{W}\right]$ which was active for the entire period of observation. There is no clear patera feature in this region (see Figure 6F), nor was activity detected here during the Galileo or New Horizons missions, apart from an HST NICMOS detection ${ }^{6}$ during the Galileo mission at $60 \pm 15^{\circ} \mathrm{N}, 60 \pm 15^{\circ} \mathrm{W}$ (Lopes et al., 2007), which is consistent with this location within the large uncertainties. However, de Pater et al. (2014b) detected emission at this site from ground-based observations in 2010, suggesting that activity in this location began between 2007 and 2010 and continued for 5 or more years, either continuously or in episodes.

The location of the hot spot PV59 $\left[\mathbf{3 8}^{\circ} \mathbf{S} \mathbf{2 9 0}^{\circ} \mathbf{W}\right]$ is well constrained, and is more consistent with the smaller dark-floored patera (PV59) a few degrees northwest of Ulgen Patera than with Ulgen Patera itself (PV56; see Figure 6AK). Past activity in this region has been attributed to Ulgen Patera; however, the location of PV59 is also consistent within uncertainties with the activity detected by NIMS and attributed to Ulgen Patera (Lopes et al., 2007), and is also closer than Ulgen Patera to the emission detected by New Horizons (Tsang et al. 2014). It is possible that this smaller patera has been the source of some or all of activity previously attributed to Ulgen Patera.

We detected faint activity at the following five hot spots where activity has never, or very rarely, been seen before: Pfu374 $\left[25^{\circ} \mathbf{S} 49^{\circ} \mathrm{W}\right]$, which we attribute to a light-floored patera at this location (see Figure $6 \mathrm{D})$; Shango Patera $\left[\mathbf{3 3}^{\circ} \mathbf{N} \mathbf{9 7}^{\circ} \mathbf{W}\right]$; Pfu1063 $\left[\mathbf{4 1}^{\circ} \mathbf{N}, \mathbf{3 5 5}^{\circ} \mathbf{W}\right]$, where the emission may originate from a light patera at $43^{\circ} \mathrm{N}, 357^{\circ} \mathrm{W}$ (see Figure $6 \mathrm{AU}$ ); and Paive Patera $\left[\mathbf{4 2}^{\circ} \mathbf{S}, \mathbf{0}^{\circ} \mathbf{W}\right.$ ]. Paive Patera was also detected on Io in eclipse in May 2008 (de Pater et al., 2016c). $\mathbf{P 2 0 7}\left[\mathbf{3 7}^{\circ} \mathbf{S}, \mathbf{9 1}{ }^{\circ} \mathbf{W}\right]$ is a small dark-floored patera, where emission was not detected by Galileo but was seen with Keck in 2001 (Marchis et al., 2005).

The activity in $\mathbf{N}$ Lerna Regio $\left[\mathbf{5 7}^{\circ} \mathbf{S}, \mathbf{2 8 9}^{\circ} \mathbf{W}\right]$ may originate from a patera at this location (see Figure $6 \mathrm{AL}$ ), and was also seen by Galileo and New Horizons. The hot spot identified as $\mathbf{P V 1 7 0}\left[\mathbf{4 8}^{\circ} \mathbf{S}, \mathbf{3 2 8}^{\circ} \mathbf{W}\right]$ may be associated with a bright-floored patera just west of Argos Planum, at $49^{\circ} \mathrm{S}, 330^{\circ} \mathrm{W}$ (see Figure $6 \mathrm{AS}$ ).

Activity at East Girru $\left[\mathbf{2 1}^{\circ} \mathbf{N}, \mathbf{2 3 4}^{\circ} \mathbf{W}\right.$ ] was detected for the first time by New Horizons in 2007 (Tsang et al., 2014; Rathbun et al., 2014). We detected brief activity at this location in December 2013; if this emission originates from the same source, this means that activity started between the Galileo mission and 2007, and continued up through 2013, either continuously or in sporadic eruptions. We did not detect further activity at this site after December 2013.

\subsection{Eruption at Kurdalagon Patera}

An unusual eruption took place in the spring of 2015 at Kurdalagon Patera. We first detected activity here on Jan 26, 2015; Kurdalagon Patera was bright on this date despite being below detection limits less

\footnotetext{
${ }^{6} \mathrm{~J}$. Spencer and R. Howell
} 


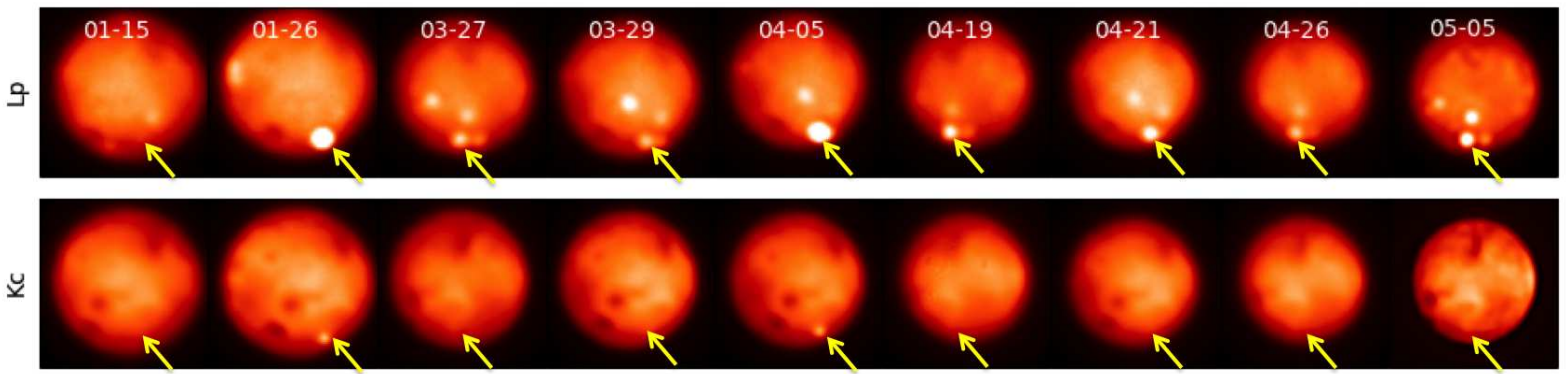

Figure 7: Observations of the progression of eruptions at Kurdalagon Patera in 2015. Arrows indicate location of Kurdalagon Patera. 

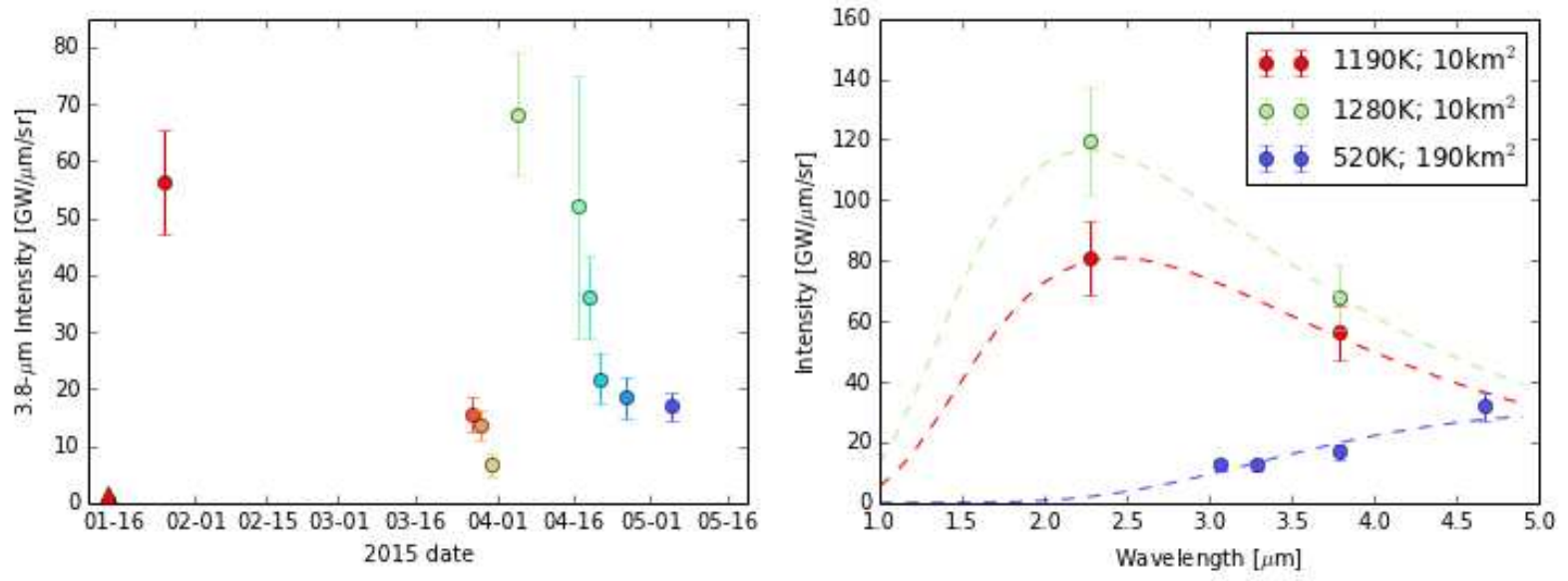

Figure 8: (Left) Timeline of 3.8- $\mu \mathrm{m}$ emission at Kurdalagon Patera in 2015. (Right) Best-fit temperature models on the dates when activity was detected at multiple wavelengths. Colors of points correspond between the two plots.

\subsection{Diminished Activity at Pele}

Pele $\left[\mathbf{1 8}^{\circ} \mathbf{S}, \mathbf{2 5 5}^{\circ} \mathbf{W}\right]$ was consistently active on Io's disk throughout the Galileo mission (Davies et al., 2012), and maintained this level of activity through at least 2002, although its intensity dropped to $25 \%$ of its former level in 2011-2014 (de Pater et al. 2016b). In 2013-2015, Pele was consistently detected by Keck at low intensity whenever the geometry was favorable, but fell consistently below the detection threshold at Gemini N. These low flux densities confirm that Pele's period of low activity that began by 2011 continued through the end of 2015 .

\subsection{Variability at Amirani}

Amirani $\left[\mathbf{2 1}^{\circ} \mathbf{N}, \mathbf{1 1 4}^{\circ} \mathbf{W}\right]$ is the largest active lava flow field in the Solar System, and was detected consistently as a thermal source by Galileo NIMS. Davies et al. (2014) analyzed variability in emission at Amirani from NIMS observations, noting that the position of effusive activity changed over time, with a decreased intensity in 2001, which these authors attribute to magma depletion following an outburst event in February 2001 (Marchis et al., 2002). The level of activity we observe at Amirani is a factor of a 2 lower than the 2001 activity, and there is no major time-variability in our period of observation. As in the NIMS dataset, we find that the position of thermal emission changes between observations, possibly due to multiple active hot spots that we are unable to resolve. However, while Davies et al. (2014) note mainly north-south differences in hot spot locations, we detect emission varying longitudinally only at the southern end of the flow field (see Figure 6P) near these authors' position 5, where only low-level activity was observed by NIMS. These position changes are consistent with the description of Amirani as a network of lava flows fed by lava tubes that extend for hundreds of kilometers, where thermal emission is observed where lava breaks out at the end of or along the lava tube system (Keszthelyi et al. 2001; Davies et al. 2014). 
1 4.6. Loki Patera

2 We detected activity at Loki Patera $\left[\mathbf{1 2}^{\circ} \mathbf{N}, \mathbf{3 1 0}^{\circ} \mathbf{W}\right]$ every time the geometry was favorable, a total of 346 times during our campaign. This site is often the brightest hot spot on Io's disk, and has exhibited regular 4 brightening episodes for decades; a model of Loki Patera as an overturning lava lake (Rathbun et al., 2002) 5 has provided a good fit to past observations (Rathbun et al., 2006; Matson et al., 2006). We detected three

6 brightening episodes in 2013-2015, which are analyzed in the context of the Matson et al. (2006) "magma 7 sea" model in a companion paper (de Kleer and de Pater, 2016b).

\section{5. Discussion}

\section{5.1. Comparison with past activity}

10 The majority of the hot spots where we detected activity have been previously detected by spacecraft, 11 indicating that sites typically stay active (or episodically resurge) for decades or longer. We detected activity 12 at five sites that were not detected during the Galileo mission: PV170, Pfu1063, Paive Patera, East Girru, 13 and Chalybes Regio. Activity at most of these sites was detected three or less times during our program, 14 and three were detected only at Ms-band $(4.7 \mu \mathrm{m})$. East Girru was first detected by New Horizons (Spencer 15 et al., 2007), and exhibited high time variability during these observations (Rathbun et al. 2014). We 16 detected activity here three times in December 2013, when it exhibited moderate L'-band intensities. Table

171 compares the locations where we detected emission with past detections by the Galileo (or ground-based 18 support) and New Horizons missions, and whether any plume has been seen in the past.

Table 1: Hot Spot Activity Summary

\begin{tabular}{|c|c|c|c|c|c|c|c|c|c|}
\hline ID & $\begin{array}{l}\text { Candidate } \\
\text { Surface Feature }\end{array}$ & $\overline{\mathrm{P}, \mathrm{PV}, \mathrm{Pf}^{a}}$ & $\begin{array}{l}\text { Approx. } \\
\mathrm{Lat}^{b}\left[{ }^{\circ} \mathrm{N}\right]\end{array}$ & $\begin{array}{l}\text { Approx. } \\
\operatorname{Lon}^{b}\left[{ }^{\circ} \mathrm{W}\right]\end{array}$ & $\begin{array}{l}F_{3.8, \max } \\
{[\mathrm{GW} / \mu \mathrm{m} / \mathrm{sr}]}\end{array}$ & $\begin{array}{l}\mathrm{N}_{\text {det }} \\
{[\#]}\end{array}$ & $\begin{array}{l}\text { Detected during } \\
\text { Galileo era? }\end{array}$ & $\begin{array}{l}\text { Detected by } \\
\text { New Horizons? }\end{array}$ & $\begin{array}{l}\text { past plume } \\
\text { activity? }\end{array}$ \\
\hline A & Uta Patera & PV183 & $-34.4 \pm 0.9$ & $22.8 \pm 2.1$ & 5.1 & 21 & Keck & - & no \\
\hline B & Kanehekili Fluctus & Pfu1730 & $-16.8 \pm 1.2$ & $33.8 \pm 1.9$ & 1.5 & 7 & NIMS, SSI & LEISA, LORRI & yes \\
\hline $\mathrm{C}$ & Janus Patera & Pfu1723 & $-4.3 \pm 0.6$ & $38.3 \pm 1.8$ & 10.7 & 32 & NIMS, SSI & LEISA & no \\
\hline D & Pfu374 & Pfu374 & $-24.6 \pm 0.8$ & $49.2 \pm 1.9$ & $<1$ & 3 & - & - & no \\
\hline $\mathrm{E}$ & Masubi Fluctus & PFd1737 & $-42.3 \pm 0.6$ & $55.1 \pm 1.5$ & 2.8 & 5 & NIMS, SSI & LEISA & yes \\
\hline $\mathrm{F}$ & Chalybes Regio & - & $55.7 \pm 2.0$ & $68.3 \pm 4.5$ & 9.8 & 22 & HST $?^{f}$ & - & no \\
\hline G & Zal Patera & P182 & $38.3 \pm 1.5$ & $74.4 \pm 2.8$ & 4.6 & 19 & NIMS, SSI & LEISA, LORRI & yes \\
\hline $\mathrm{H}$ & Tawhaki Patera & P229 & $3.2 \pm 0.7$ & $75.8 \pm 1.3$ & 4.5 & 11 & NIMS, SSI & LEISA & no \\
\hline I & Aluna Patera & P192 & $41.7 \pm 1.3$ & $89.9 \pm 1.6$ & $<1$ & 2 & SSI, NIMS 131E & - & no \\
\hline $\mathrm{J}$ & Gish Bar Patera & P178 & $15.2 \pm 0.6$ & $89.0 \pm 1.5$ & 7.3 & 13 & NIMS, SSI & - & no \\
\hline K & P207 & P207 & $-36.5 \pm 1.2$ & $91.1 \pm 0.8$ & 5.0 & 1 & Keck & - & no \\
\hline $\mathrm{L}$ & Shango Patera & P179 & $33.2 \pm 0.9$ & $96.9 \pm 2.9$ & $<1$ & 3 & NIMS & - & no \\
\hline M & Sigurd Patera & P219 & $-4.9 \pm 1.0$ & $98.3 \pm 2.1$ & 5.9 & 8 & NIMS & - & no \\
\hline $\mathrm{N}$ & Itzamna Patera & P221 & $-14.4 \pm 0.6$ & $98.9 \pm 0.9$ & 2.6 & 4 & NIMS & - & no \\
\hline $\mathrm{O}$ & P197 & P197 & $-46.5 \pm 1.7$ & $107.3 \pm 2.1$ & 11.6 & 11 & NIMS I32G & - & no \\
\hline $\mathrm{P}$ & Amirani & Fd2021 & $20.9 \pm 1.0$ & $113.7 \pm 2.8$ & 4.1 & 12 & NIMS, SSI, Keck & LEISA, LORRI & yes \\
\hline Q & Dusura Patera & P161 & $36.2 \pm 0.8$ & $121.6 \pm 1.3$ & 8.1 & 3 & NIMS & - & no \\
\hline $\mathrm{R}$ & Malik Patera & P120 & $-33.0 \pm 1.2$ & $129.4 \pm 1.8$ & 6.9 & 8 & NIMS & LEISA & no \\
\hline $\mathrm{S}$ & Thor & - & $40.4 \pm 1.2$ & $135.0 \pm 1.6$ & $<1$ & 2 & NIMS & LEISA & yes \\
\hline $\mathrm{T}$ & P123 & P123 & $-41.6 \pm 0.6$ & $139.8 \pm 3.2$ & 4.5 & 3 & NIMS I32H/I & - & no \\
\hline $\mathrm{U}$ & Tupan Patera & P97 & $-17.8 \pm 0.5$ & $140.1 \pm 2.8$ & 1.6 & 3 & NIMS & LEISA & no \\
\hline V & Surya Patera & P55 & $21.3 \pm 0.5$ & $149.3 \pm 0.9$ & 3.5 & 4 & NIMS & & yes \\
\hline
\end{tabular}




$\begin{array}{llllll}\text { W } & \text { Prometheus } & \text { P86 } & -1.0 \pm 0.7 & 153.6 \pm 2.0 & 5.3 \\ \text { X } & \text { Culann (NW) } & \text { Fd1891 } & -16.2 \pm 0.8 & 162.3 \pm 3.3 & 2.1 \\ \text { Y } & \text { Sethlaus/Gabija Paterae } & \text { P134/P136 } & -50.0 \pm 0.8 & 198.2 \pm 1.2 & 32.6 \\ \text { Z } & \text { Isum Patera } & \text { P170 } & 31.9 \pm 0.4 & 209.2 \pm 0.5 & <1 \\ \text { AA } & \text { Marduk Fluctus } & \text { P32 } & -23.9 \pm 1.3 & 210.7 \pm 3.4 & 9.1 \\ \text { AB } & \text { Kurdalagon Patera } & \text { P142 } & -48.8 \pm 1.4 & 221.3 \pm 2.8 & 68.1 \\ \text { AC } & \text { Susanoo/Mulungu Paterae } & \text { P2/P1 } & 18.8 \pm 1.6 & 221.9 \pm 2.4 & 3.7 \\ \text { AD } & \text { 201308C } & - & 29.0 \pm 1.0 & 229.8 \pm 2.5 & >500 \\ \text { AE } & \text { East Girru } & - & 21.4 \pm 0.8 & 233.5 \pm 1.2 & 5.3 \\ \text { AF } & \text { Pyerun Patera } & \text { PV26 } & -57.7 \pm 1.9 & 237.1 \pm 2.3 & <1 \\ \text { AG } & \text { Pillan Patera } & \text { P147 } & -11.5 \pm 0.4 & 245.4 \pm 1.5 & 7.5 \\ \text { AH } & \text { Chors Patera } & - & 64.8 \pm 1.2 & 246.7 \pm 3.7 & 56.7 \\ \text { AI } & \text { Pele } & \text { PV30 } & -18.1 \pm 1.0 & 255.0 \pm 2.5 & 6.9 \\ \text { AJ } & \text { Mithra Patera } & \text { PV39 } & -58.1 \pm 0.9 & 266.2 \pm 2.4 & 54.5 \\ \text { AK } & \text { PV59 } & \text { PV59 } & -38.4 \pm 1.5 & 290.0 \pm 2.4 & 17.5 \\ \text { AL } & \text { N Lerna Regio } & - & -57.3 \pm 1.2 & 288.8 \pm 5.3 & 7.1 \\ \text { AM } & \text { Amaterasu Patera } & \text { PV78 } & 39.4 \pm 0.8 & 304.3 \pm 1.1 & 43.1 \\ \text { AN } & \text { Rarog Patera } & \text { PV73 } & -38.7 \pm 2.0 & 306.0 \pm 2.6 & 325 \\ \text { AO } & \text { Heno Patera } & \text { PV85 } & -56.0 \pm 1.3 & 307.2 \pm 1.8 & 270 \\ \text { AP } & \text { Loki Patera } & \text { PV81 } & 12.0 \pm 1.0 & 308.2 \pm 4.2 & 136 \\ \text { AQ } & \text { Tol-Ava Patera } & \text { PV106 } & 0.7 \pm 0.7 & 326.2 \pm 1.2 & 8.3 \\ \text { AR } & \text { Fuchi Patera } & \text { PV122 } & 28.3 \pm 1.1 & 328.7 \pm 1.1 & 1.0 \\ \text { AS } & \text { PV170 } & \text { PV170 } & -47.8 \pm 1.6 & 327.9 \pm 1.8 & 11.6 \\ \text { AT } & \text { Surt } & \text { PV131 } & 44.3 \pm 1.3 & 334.0 \pm 2.3 & <1 \\ \text { AU } & \text { Pfu1063 } & \text { Pfu1063 } & 40.5 \pm 1.7 & 355.2 \pm 2.4 & 2.3 \\ \text { AV } & \text { Paive Patera } & \text { PV171 } & -42.1 \pm 1.5 & 359.6 \pm 1.6 & <1\end{array}$

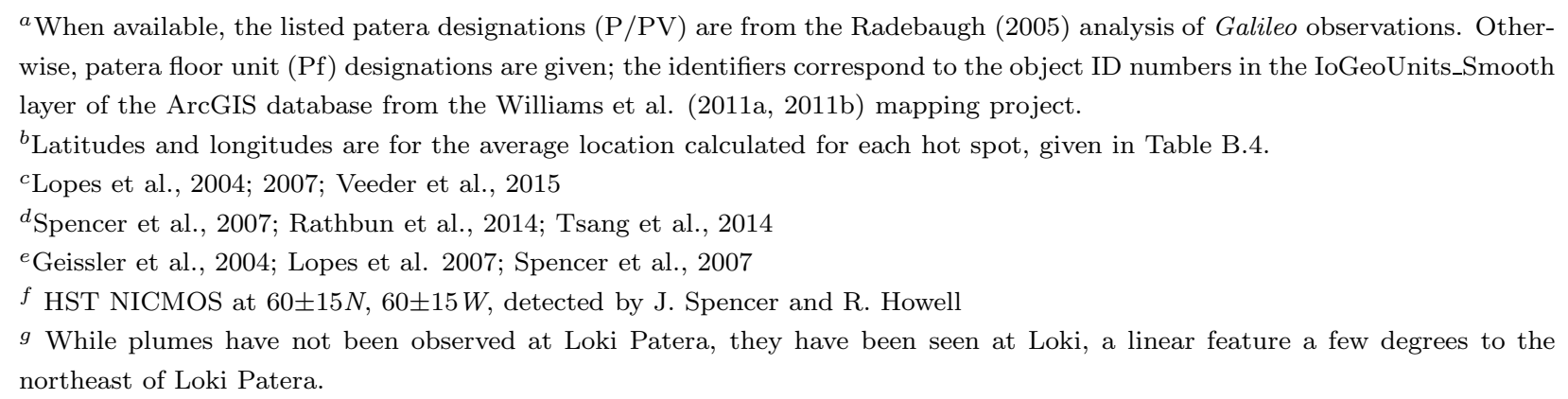

Of the new hot spots, only Chalybes Regio was detected consistently throughout the period of observation. 13 Activity here was not detected by Galileo or New Horizons, though an HST NICMOS detection during the

Galileo mission with errorbars of $\pm 15^{\circ}$ in latitude and longitude (Lopes et al., 2007) is consistent with its location. Apart from this, the first documented activity at this location was in 2010 Keck observations (de Pater et al., 2014b). There are no apparent features indicative of recent volcanic activity in spacecraft images, though the region is full of undivided and bright flows (Williams et al., 2011a; 2011b), indicating activity in this area in the distant past. In addition, there was no evidence for surface changes in this region in New Horizons images from 2007 (Spencer et al., 2007). It appears that this is a new active hot spot, or an older volcanic center becoming active again after a long interval of inactivity. A future detection of new flows or deposits in this region by spacecraft or high-resolution visible imaging would substantiate this possibility.

The LEISA instrument $(1.25-2.5 \mu \mathrm{m})$ detected 37 active hot spots during the New Horizons fly-by of the Jupiter system in 2007 (Tsang et al. 2014); 90\% of these were active during the Galileo era, indicating 
that most hot spots are active on decadal timescales. Of the 37 hot spots detected during the New Horizons fly-by, we detected activity at only 25 (67\%) of them. If activity at the remaining sites continued through 2015, their emission was below our detectability threshold.

While the same hot spots appear to stay active on Io for decades, the relative intensities at different locations varies greatly. Veeder et al. (2015) identify the least powerful hot spot measurable from Galileo data as P197, and the most powerful eruption during the same years as Surt (measured from the ground by Keck; Marchis et al., 2002). While we detected thermal emission from both sites during our program, P197 stayed active at a moderate level of activity for over a year, while Surt was barely detected twice, and only at $4.7 \mu \mathrm{m}$. We also detected no activity at Tvashtar Paterae, which has exhibited bright outbursts in the past (Howell et al., 2001; Milazzo et al., 2005), and was the most powerful event observed in 2006-2007, detected from the ground and by New Horizons (Laver et al., 2007; Spencer et al., 2007; Rathbun et al., 2014). Other sites that have exhibited past bright eruptions include Kanehekili Fluctus and Pillan Patera (detected from the ground: Marchis et al., 2002; de Pater et al., 2014; de Pater et al., 2016a, and by Galileo: Keszthelyi et al., 2001; Davies et al., 2001), which were all at or below our detection threshold in 2013-2015, until the eruption at Pillan Patera in Feb 2015 (de Pater at al., 2016a). It appears that the sites that host the most powerful eruptions also exhibit the greatest time variability, often remaining below the detection threshold for long periods of time before brightening abruptly. In contrast, sites such as Marduk Fluctus and Janus Patera have held steady at low to moderate brightness for decades without exhibiting such brightening events. This difference can be understood in terms of the longer period of repose necessary to recharge a system after a major eruption with a large volume discharged, before another eruption is possible (Davies 2007).

\subsection{Eruption classification}

In our dataset, we find a clear distinction between two observational classes of hot spots: those that exhibit brief activity involving a pattern of sudden appearance and decay, and those that exhibit long-lived activity with low time variability and moderate intensities. The timelines of the hot spots in each class are compared in Figure 9. We define and characterize these two eruptions classes as follows:

1. Hot spots exhibiting sudden brightening events: These hot spots are typically below the detectability threshold until a sudden increase in brightness, which is followed by a steady decay typically over a timescale of $\sim 1$ month. The peak L'-band brightness are 5-10 $\mathrm{GW} / \mu \mathrm{m} / \mathrm{sr}$ or higher, and may even exceed 500 $\mathrm{GW} / \mu \mathrm{m} / \mathrm{sr}$. Every single detection of an L'-band intensity over $15 \mathrm{GW} / \mu \mathrm{m} / \mathrm{sr}$ in our dataset is associated with such a brightening event. The 16 hot spots included in this category are listed in Table 2.

2. Persistent hot spots: Hot spots in this category were consistently active for $>1$ year (and usually 
Table 2: Classification of Hot Spots

\begin{tabular}{|c|c|c|c|c|}
\hline Persistent Activity & \multicolumn{3}{|c|}{ Sudden Brightening Events } & Both \\
\hline & Outbursts & Mini-Outbursts $^{a}$ & Other & \\
\hline $\begin{array}{c}\text { Marduk Fluctus } \\
\text { Uta Patera } \\
\text { Janus Patera } \\
\text { Chalybes Regio } \\
\text { Zal Patera } \\
\text { Tawhaki Patera } \\
\text { Gish Bar Patera } \\
\text { Amirani } \\
\text { Prometheus } \\
\text { Pele }\end{array}$ & $\begin{array}{c}\text { Rarog Patera } \\
\text { Heno Patera } \\
\text { 201308C }\end{array}$ & $\begin{array}{c}\text { Sethlaus/Gabija Paterae } \\
\text { Kurdalagon Patera } \\
\text { Chors Patera } \\
\text { Mithra Patera } \\
\text { Pillan Patera } \\
\text { Amaterasu Patera }\end{array}$ & $\begin{array}{c}\text { Sigurd Patera } \\
\text { East Girru } \\
\text { PV59 } \\
\text { Malik Patera } \\
\text { Dusura Patera } \\
\text { PV170 } \\
\text { Tol-Ava Patera }\end{array}$ & $\begin{array}{c}\text { Loki Patera } \\
\text { P197 }\end{array}$ \\
\hline
\end{tabular}

${ }^{a}$ See Section 5.6.

1 for the entire duration of the program), and were detected whenever the geometry was favorable during 2 their period of activity. They exhibit low time-variability, typically remaining within a factor of a few in brightness. They also maintain a low to moderate L'-band brightness; no detection of a consistently-detected hot spot exceeds $15 \mathrm{GW} / \mu \mathrm{m} / \mathrm{sr}$. This category includes the 10 hot spots listed in Table 2.

Figure 9: Comparison of thermal emission timelines for the two classes of hot spots defined in Section 5.2. The two classes are clearly distinguished by eye in terms of the peak brightness obtained, and the duration of activity. The two hot spots that fall into both categories (P197 and Loki Patera) are omitted.

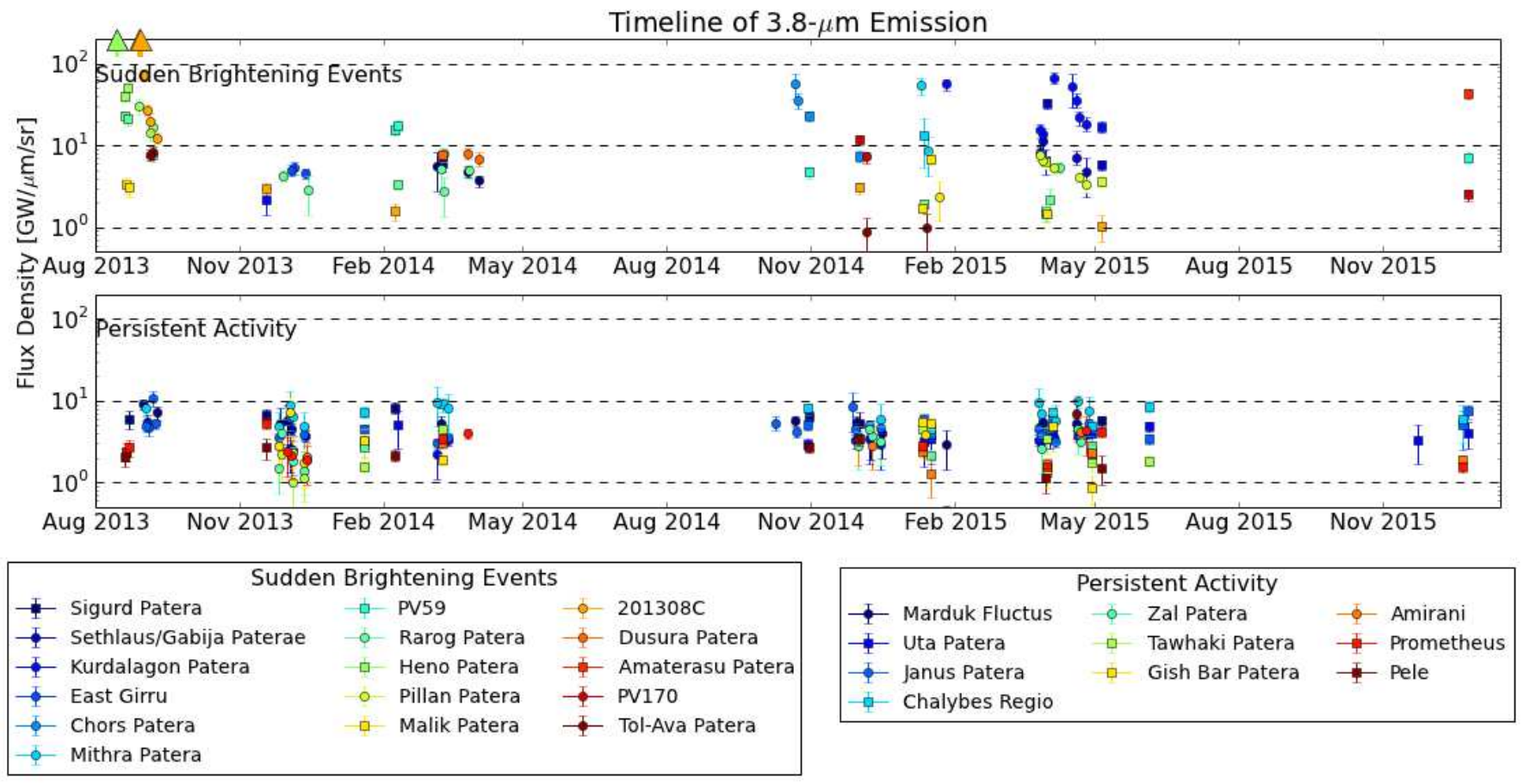

$5 \quad$ Loki Patera and P197 exhibit eruption events despite being persistently active, and we consider them in 6 both categories. An additional $20+$ hot spots were detected too few times to confidently classify them into 
either category. While bright eruptions preferentially occur at high latitudes and on the trailing hemisphere, we see long-lived eruptions of moderate brightness located preferentially at equatorial latitudes and on the leading hemisphere. This phenomenon is analyzed in de Kleer and de Pater (2016a).

The distinction between hot spots characterized by persistent (months to years) or sporadic (short-lived events) activity was also present in the Galileo dataset, as discussed in Lopes-Gautier et al. (1999). While these authors find hot spots to be evenly distributed on the surface, they also find persistent hot spots located preferentially at equatorial latitudes.

We identify 10 persistent hot spots, while 22 were identified in Galileo NIMS data (Lopes-Gautier et al., 1999). We note that our definition requires persistent hot spots to be consistently detected, while these authors only require detections over a $>1$ year period. If we adopt the latter definition, we find that of the 22 long-lived spots identified in the NIMS data, 16 of them were also active for $>1$ year in our dataset. In addition, of the 15 bright, sporadic hot spots identified in our dataset, only three were active for 1 year or more during the Galileo mission.

This consistency over decadal timescales supports the hypothesis that eruption characteristics represent physically-distinct eruption styles (Davies et al. 2010; Veeder et al. 2012). Davies et al. (2010) present a method to distinguish between different eruption styles based on their thermal signatures, in particular the time-evolution of their 2-to-5- $\mu$ m radiant flux densities, based on a comparison with thermal signatures of volcanism on Earth. We do not typically detect thermal emission at $2 \mu \mathrm{m}$ and in general cannot interpret our observations using this metric. However, the temporal variability and peak brightnesses can be used to broadly associate persistent hot spots with insulated flow fields or lava lakes, and hot spots with brightening events as lava fountains, open sheet flows, or break-outs in lava flow fields (Davies et al. 2010).

\subsection{Searching for periodicity in volcanic activity level}

Io's volcanic activity is powered by periodic tidal forcing from Jupiter, and we might therefore expect to see periodic variations in Io's activity level with its orbit, or on longer timescales associated with secondary factors such as the orbits of Europa and Ganymede, or possible obliquity tides such as has been hypothesized for Europa's plumes (Rhoden et al., 2015). However, we note that the periodic forcing is modulated by many geological factors, and will not necessarily lead to periodic volcanic activity.

We search for periodic behavior in the level of volcanic activity on Io, parameterized by the total volcanic L'-band emission on each date of observation. We employ a generalized Lomb-Scargle analysis (Scargle 1982; Zechmeister and Kürster, 2009), which is designed for frequency analysis of unequally-spaced timeseries data. The generalized Lomb-Scargle periodogram is shown in Figure 10 including the 50\% and 90\% significance thresholds. We do not find significant periodicity at Io's orbital period or any multiples thereof. 
Figure 10: Generalized Lomb-Scargle periodogram for total volcanic L'-band emission as a function of date, separated into all data (top), and data with central meridian longitude $(\mathrm{cml})$ above or below $180^{\circ}$, indicative of orbital phase. The middle panels show a zoom-in of the periods between 0-20 days, and the right panels show the data phased to 14.937 days, the strongest signal in the periodogram. The dashed lines indicate confidence levels.
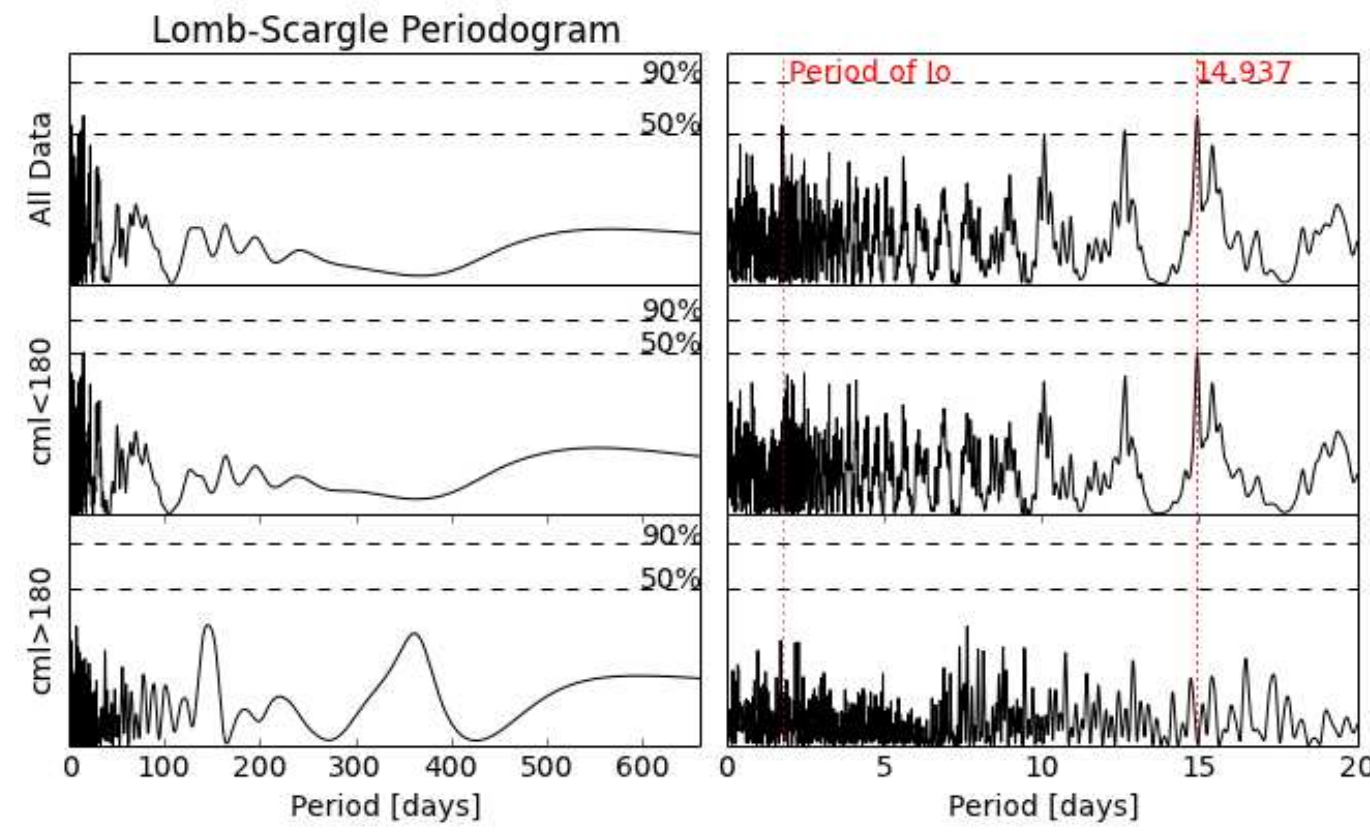

The cluster of low-significance peaks in this vicinity may be due in part to the fact that bright eruptions may last for many days, and observations separated by an interval near Io's period will catch the same eruption.

The strongest signal we detect is at a period of 14.937 days. Figure 10 shows the data phased to this period, and demonstrates that the periodicity is due almost exclusively to observations during the Earthapproaching phase of Io's orbit. It is difficult to distinguish trends in volcanic activity with orbital phase from trends with longitude, since (from Earth) the same longitudes are always observed at the same orbital phase. However, observations at times far from either opposition sample both leading and trailing hemispheres simultaneously, and can help distinguish between these two scenarios. If we consider only hot spots in the leading hemisphere of Io, the noted periodicity is not present, while when we consider only observations in the leading phase of Io's orbit (as is shown in Figure 10) it is apparent. If this trend is real, it is therefore more correlated with orbital phase than with the longitudes of hot spots.

We consider this signal tentative; additional observations will be needed to confirm its validity, and in particular observations which trace Io's total volcanic heat flow rather than intensity at a single wavelength.

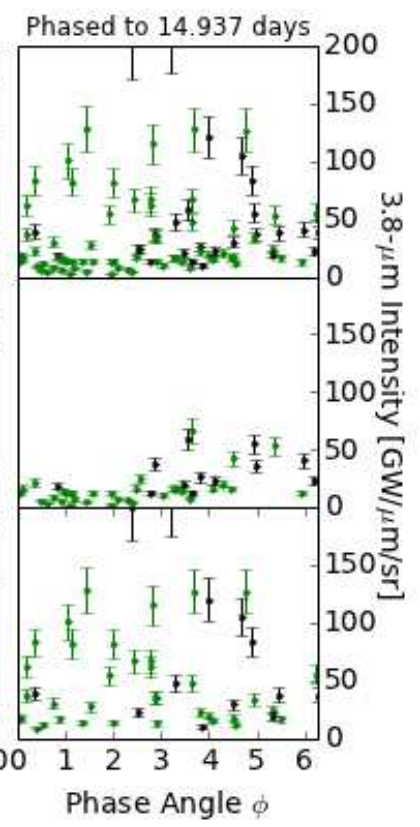

\subsection{How long do eruptions last?}

Constraints on typical eruption durations are crucial for constraining the contribution of an individual hot spot to Io's total heat flow, and for determining the physical mechanisms at play. Of the hot spots where 
Figure 11: Comparison of eruption timelines for all hot spots that exhibited "eruption phases", defined as a sudden brightening followed by a steady decay. The timelines demonstrate the evolution of emission at a wavelength of $3.8 \mu \mathrm{m}$.

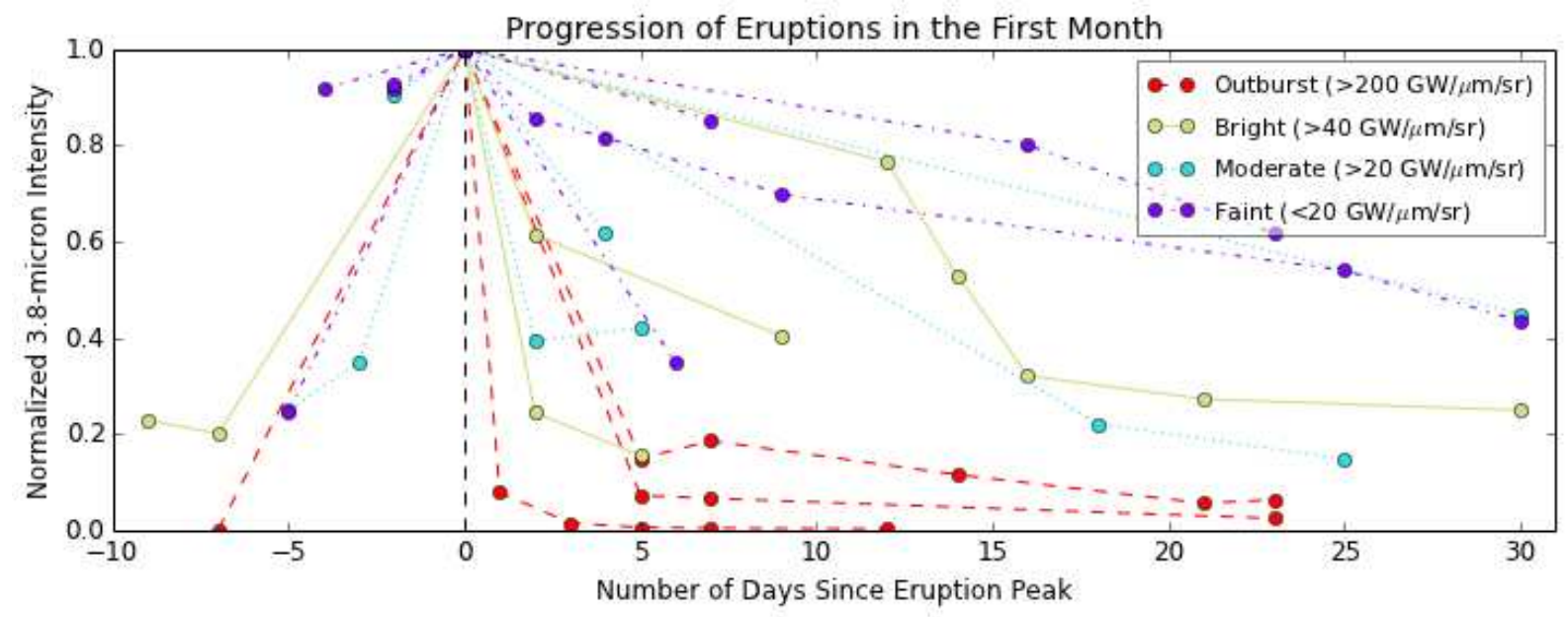

we detected thermal activity, a quarter of them stayed active for a year or more, at moderate intensity and with only moderate time variability (within a factor of a few). Another third exhibited discrete eruption phases, where the emission either appeared or increased rapidly, followed by a monotonic decline in intensity. In nearly all cases, this decline occurred over a matter of days to weeks; we find that with the exception of the outburst eruptions, most events have declined to half their peak detected level after 10-30 days. The three outbursts we detected decreased in intensity by a factor of 10 in a matter of days, probably marking the transition from lava fountaining to effusive flows (de Kleer et al., 2014; de Pater et al., 2016a). Figure 11 compares the timelines for appearance and decay of all the bright discrete eruption events detected. The rate of decay appears somewhat correlated with the peak intensity. However, this may be an artifact arising from the fact that we generally do not capture the actual peak eruption intensity.

Of the remaining events, several were detected only a few times in a period of 1-3 months, and the rest were either too near the detection threshold or detected too few times to draw conclusions about their durations.

\subsection{Power output of different eruption classes}

The total time-averaged power of a given hot spot is dictated by both the power emitted when active, and the duration of activity. In most cases, the duration of activity is a better predictor of total power than the peak power emitted. While a low-power hot spot may generate only $100 \mathrm{GW}$ compared to the peak outburst powers of $50+$ TW, the time-averaged power of a smaller, continuously-active hot spot will still dominate over that of the outburst. Indeed, Veeder et al. (2012) estimate that although outbursts are the most powerful class of eruptions on Io, they contribute less than $4 \%$ of the total heat flow from Io's surface because they are both infrequent and short-lived. We can only constrain the power output for $~ 30 \%$ of 
our detections, and therefore cannot quantify the distribution of volcanic heat flow in our observations with precision. However, we make a rough estimation based on the measurements where we do constrain power. We take an average power output of $200 \mathrm{GW}$ for moderate, long-lived hot spots, and an average power of 1 TW over a 15-day period for eruption events. Using these numbers, a moderate long-lived hot spot will contribute $\sim 5 \mathrm{x}$ as much to Io's total heat flow as a violent but short eruption. Although we detect $50 \%$ more hot spots exhibiting eruption events than persistent hot spots, we estimate based on these numbers that long-lived hot spots dominated Io's volcanic heat flow during 2013-2015.

\subsection{Frequency and Timing of Large Eruptions}

Due to the very low number of outburst events ever detected on Io, it has been difficult to estimate the frequency of their occurrence and to characterize their properties. An outburst is defined empirically as an event which doubles Io's total 5- $\mu$ m intensity (Blaney et al., 1995; Spencer and Schneider, 1996). Based on 55 nights of observation, Veeder et al. (1994) estimated that outbursts occur $4 \%$ of the time. The occurrence of three outburst events in a two-week period in August 2013 suggested that such events may be more common than this, and led de Pater et al. (2014) to update the estimate to $10 \%$ for the chance of detecting an outburst on a given night, based on 75 nights of observation. However, following these three outburst events in August 2013, no others were detected during the remaining 90+ nights of our observing period, although it is possible that some of the eruptions we detected which were bright but not of outburst scale may have been outburst events that we did not detect until they had passed the brightest phase of the eruption.

We identify a second class of bright "mini-outburst" eruptions, whose peak 3.8- $\mu$ m emission is a factor of $\sim 5$ too low to qualify as outburst eruptions, but whose eruption characteristics resemble those of outbursts. These characteristics include a sudden appearance of emission, rapid decay, high peak brightness, and high short-wavelength emission (when measured). We identify five such eruptions in our dataset, all of which appeared abruptly, exhibited rapid time-variability, and reached peak $3.8-\mu \mathrm{m}$ intensities of $30-80 \mathrm{GW} / \mu \mathrm{m} / \mathrm{sr}$ : Kurdalagon Patera, Chors Patera, Mithra Patera, Sethlaus/Gabija Paterae, Amaterasu Patera. In the case of Kurdalagon Patera, we also detect high thermal emission at $2.3 \mu \mathrm{m}$.

These "mini-outbursts" may be caused by a similar physical mechanism as outburst eruptions operating on a smaller scale, i.e. lava fountains and open channel flows (Davies et al. 2010). Eruptions in this "minioutburst" class have been seen before, for example at Tung Yo Patera and Sui Jen Patera in 2004; South of Babbar Patera in 2005 (de Pater et al. 2016b); and Kanehekili Fluctus in 2010 (de Pater et al. 2014b). All four of these eruptions had peak $3.8-\mu \mathrm{m}$ intensities in the same range as those of our dataset.

The occurrence of three outburst eruptions within a two week period, followed by a lack of detections in 
the subsequent two years, indicates that either the outburst frequency estimate needs to be lowered again and the three events detected in August were a rare coincidence, or that outbursts are correlated in time. There is some precedent for outburst events occurring closely-spaced in time. In 1996, two outbursts were detected just over a month from one another, at 16 and $35^{\circ} \mathrm{W}$ (Stansberry et al., 1997). Two outbursts were also detected within 3 days of one another in February 2001, at Amirani and Surt (at 118 and $340^{\circ}$ W), following only two months after an outburst at Tvashtar in December 2000 (at 120W) (Marchis et al., 2002; Milazzo et al., 2005).

A similar effect is seen within the "mini-outburst" class. The eruptions at Kurdalagon Patera and Sethlaus/Gabija Paterae in Apr/May 2015 began abruptly within a few days of one another; these sites are separated by $\sim 500 \mathrm{~km}$. Nearby sites erupting within days of one another were also seen in the eruptions at Tung Yo Patera and Sui Jen Patera on 28 and 30 May 2004 (de Pater et al., 2016b). The phenomenon of volcanic eruptions occurring in pairs within a two-day period and up to a separation of $200 \mathrm{~km}$ has been observed on Earth (Linde and Sacks, 1998; Manga and Brodsky, 2006; Williams 1995), although the mechanisms are not well understood. The correlation disappears at a separation greater than $200 \mathrm{~km}$, suggesting either a regional seismic trigger for both eruptions, or the triggering of one volcano by the other due to eruption-generated earthquakes (Manga and Brodsky, 2006). Triggering of eruptions by earthquakes has been documented up to distances of $1000 \mathrm{~km}$ or more (Hill et al. 1993). Such a mechanism could operate over longer distances on Io if eruptions generate more intense shaking, or seismic waves propagate longer distances, on Io than on Earth. However, a localized phenomenon such as a seismic trigger is difficult to invoke over the longer distances that separate some of the past outburst eruption pairs. It is clear that we still do not have a good understanding of the frequency and triggering mechanisms for these high-energy events.

\subsection{Potential impact on Jupiter system: Connection to the EXCEED Mission}

Our observations overlap with the time period during which the ISAS/JAXA EXCEED Mission on board the SPRINT-A Earth-orbiting satellite (Ueno et al., 2011; Murakami et al., 2012) was observing the Jupiter system. One of the goals of this mission is to observe the Io-Jupiter plasma system in the Extreme-UV for multiple continuous months, to investigate plasma torus phenomena including the timescales and causes of variability in the torus brightness. Because Io's volcanically-produced atmosphere is the source of plasma torus constituents, a timeline of Io's simultaneous volcanic activity is crucial for understanding the processes that bring particles and gas from Io into the plasma torus. Io's massive volcanic plumes inject gas and material into Io's primary atmosphere that may lead to a spike in the supply of neutral and ionized material to the sodium cloud and plasma torus. 
The connection between variability in Io's volcanic activity and the plasma torus brightness is complicated by the fact that Io hosts many different styles of volcanic activity, only some of which have been seen to produce plumes (e.g. Geissler et al., 2008). The intensity of an eruption alone is insufficient to predict whether a plume is present, nor is the scale of thermal emission necessarily correlated with plume emission. In addition, plumes themselves vary greatly in magnitude and in gas-to-particle ratio (McEwen and Soderblom, 1983; Johnson et al., 1995), and spacecraft observations of plumes via scattered sunlight are sensitive only to the dusty component (Geissler and Goldstein, 2004). We can nevertheless gain some insight into which active sites are most likely to host plumes based on where plumes have been observed in the past. Of the sites where we observed thermal activity, the following were seen to host plume activity by Galileo, based either on direct observation or new surface deposits: Kanehekili Fluctus, Masubi Fluctus, West Zal Patera, Amirani, Thor, Prometheus, Culann Patera, Marduk Fluctus, Pillan Patera, Pele, and Surt (Geissler et al., 2004; Geissler and MacMillan 2008). New Horizons additionally detected plume activity at N. Lerna and Kurdalagon Patera (Spencer et al., 2007). Of the sites listed, only Marduk Fluctus, Pillan Patera, and Kurdalagon Patera hosted moderate to bright thermal activity during our program. We note that past plume activity cannot be ruled out by a lack of Galileo detection, as a lack of dusty material can make a plume invisible even if it hosts significant gas emission (Johnson et al., 1995).

In early 2015, a brightening in the sodium cloud was detected from D-line emission (Yoneda et al., 2015). The event began in mid-January and peaked just past mid-March, after which the sodium cloud brightness decreased to below its pre-brightening level by April. An accompanying response was seen in the plasma torus, with an increase in $\mathrm{S}^{+}$emission intensity followed by an increase in $\mathrm{S}^{2+}$ and $\mathrm{S}^{3+}$ emission in JanuaryFebruary 2015, followed by a decay to baseline levels by mid-May (Tsuchiya et al., 2015). A second, smaller brightening of the sodium cloud was seen in April-May.

Two bright eruptions took place during this time period: at Kurdalagon Patera and Pillan Patera. Kurdalagon Patera became detectable in the NIR between January 15 and 26, and reached peak intensity twice, at the end of January and again at the beginning of April, before diminishing during April and May (see Section 4.3). The eruption at Pillan Patera was detected on Feb $18^{7}$ (de Pater et al., 2016a) and had diminished substantially by the following month. Both sites have hosted plume activity in the past.

The timing of the eruption at Kurdalagon Patera is more consistent with the onset of the sodium cloud brightening, and the second smaller brightening accompanying the second eruption at Kurdalagon strengthens this case. While the two eruptions at Kurdalagon Patera were of similar intensity and temperature, the two sodium cloud brightenings were vastly different in magnitude. However, the gas emission from volcanoes

\footnotetext{
${ }^{7}$ Detected at the IRTF by R. Howell
} 
on Earth varies greatly even within the course of a single eruption (Gerlach and McGee, 1994; Sutton and Elias, 2014), and it is possible that the first eruption at Kurdalagon Patera was accompanied by a large outgassing of volatiles, while the second was not.

We emphasize that the link between thermal emission, plume activity, and the plasma torus brightness depends heavily on many complex processes that are poorly understood, and that longitude-time coverage of Io is always incomplete. The association between these two eruptions and the sodium cloud brightening is therefore speculative, but represents a plausible scenario based on the abrupt onset of bright activity at these sites where plumes have been active in the past, coincident with the brightening of the neutral cloud and plasma torus.

\section{Conclusions}

We imaged Io in the near-infrared with adaptive optics on 100 nights from August 2013 through December 2015 at the Keck and Gemini N telescopes. This dataset represents the most complete coverage of activity at individual hot spots over a multi-year period to date. Our main conclusions are as follows:

1. Volcanic Activity Timeline: We present the positions and intensities of over 400 detections of 48 hot spots, and a timeline of global activity on Io. When observations were made at two or more wavelengths (30\% of detections), we present the estimated temperatures and powers. Io's global level of volcanic activity varies widely in time. There was widespread bright activity on Io in Aug-Sep 2013 and from Oct 2014 through the spring of 2015. However, no bright hot spots were detected from Nov 2013-May 2014 despite good coverage during much this time. A search for periodicities in the timeline of total volcanic L'-band emission finds only a tentative signal at 14.937 days, due primarily to observations during the Earth-approaching phase of Io's orbit. In particular, we do not find a strong periodicity associated with Io's orbital period or any multiple thereof.

2. Detection Limits: We perform an extensive analysis of the detection limits and retrieval uncertainties for hot spots detected with Keck/NIRC2 L' and Ms filters, and Gemini N/NIRI L' filter. Based on this analysis, we establish upper limits on hot spot non-detections and quantify the accuracy of intensity and position retrievals as a function of flux density and emission angle. These are incorporated into our own results, and can be used by others in future observation planning and analysis.

3. Eruption Classifications: We classify the detected hot spots into two observationally-distinct categories: (1) Hot spots exhibiting eruption events, which are highly time-variable and reach a peak L'-band intensity of $5-10 \mathrm{GW} / \mu \mathrm{m} / \mathrm{sr}$ or more, and often greater than $50 \mathrm{GW} / \mu \mathrm{m} / \mathrm{sr}$; (2) Persistent hot spots, which are consistently detected for one year or more, exhibit low time variability, and never 
exceed an L'-band intensity of $15 \mathrm{GW} / \mu \mathrm{m} / \mathrm{sr}$. Of the 48 hot spots we detected, 16 exhibited bright eruptions, 10 were persistently active, and 2 exhibited characteristics of both categories. The remaining hot spots were too faint or detected too few times to classify. A comparison with persistent hot spots identified in the NIMS dataset (Lopes-Gautier et al., 1999) indicates that eruption characteristics often stay constant for a decade or more. Of the hot spots that exhibited bright eruptions, the activity typically began abruptly, increasing from undetectable to peak brightness in a matter of days to weeks. The timescale for decay is of the order of 10-30 days, with more rapid decay for outburst eruptions. We find that persistent hot spots dominate over bright eruption events in terms of contribution to Io's total heat flow.

4. Comparison to Past Activity: Of the 48 hot spots detected, all but five were seen during the Voyager or Galileo missions (by spacecraft or ground-based support). Of these five, most exhibited only small-scale activity, and may be associated with nearby paterae on the surface. The exceptions are East Girru, which was first detected in 2007 by New Horizons, and was active briefly with moderate intensity in 2013; and Chalybes Regio, which was consistently active throughout our observations, and was first detected in 2010 (de Pater et al., 2014b). Neither of these sources is near a volcanic surface feature, and both may represent locations of new volcanic activity since the Galileo mission. Future observations of new surface flows or deposits could confirm this possibility.

5. Outburst Eruptions: After the three outburst eruptions in August 2013 (de Kleer et al. 2014; de Pater et al. 2014a), we detected no other eruptions of outburst scale, despite observing on 90 more nights over the subsequent two years. However, we identify five "mini-outburst" eruptions, which exhibit the temporal characteristics of outburst eruptions but whose intensity is a factor of $\sim 5$ too low $(3.8-\mu \mathrm{m}$ intensities of $30-80 \mathrm{GW} / \mu \mathrm{m} / \mathrm{sr})$. These eruptions may be outburst events caught after their peak brightness, or eruptions generated by a similar physical mechanism operating on a smaller scale. The clustering of the three outburst eruptions into a two-week time period, and the occurrence of two of the mini-outbursts within a few days of one another, is intriguing. Some previous detections of large eruptions have also included multiple events in a short time period, although the total number of outburst events observed to date is too small to draw definitive conclusions.

6. Eruption at Kurdalagon Patera: We detected bright eruptions at Kurdalagon Patera on Jan 26 and April 5 of 2015, with measured temperatures of $\sim 1200 \mathrm{~K}$. Kurdalagon was not detected prior to this date, in particular 11 days earlier on Jan 15 when the viewing geometry was favorable. Between the two eruptions, activity dropped to a moderate level.

7. Implications for the Jovian system: Our observations covered a period of time when the SprintA/EXCEED Mission was observing the Io plasma torus, and ground-based support observations were 
monitoring the sodium cloud. Near the end of January 2015, a brightening of the sodium cloud and plasma torus was observed (Yoneda et al., 2015; Tsuchiya et al., 2015). This timing coincides with the sudden brightening of Kurdalagon Patera, and suggests the possibility that the brightenings may have been caused by an influx of neutral material from a plume at Kurdalagon Patera. This possibility is supported by a past detection of plume activity at Kurdalagon by New Horizons (Spencer et al., 2007). In addition, a second sodium cloud brightening of a much smaller scale occurred simultaneously with the second eruption at Kurdalagon Patera in April 2015.

These data demonstrate the continued value of frequent, ground-based observations of Io in the nearinfrared. Such observations can be very brief (<30 minutes/night) and have high scientific benefit. Between spacecraft and ground-based observations, the database of Io observations is becoming sufficiently large to begin to look at statistical properties such as periodic behaviors and the correlation of eruption properties with latitude and longitude, which are important clues for understanding the tidal forcing and heat dissipation that power Io's volcanic activity. Consistent high-cadence observations over long time baselines are needed to gain additional information on the timeline of activity, typical eruption duration and variability, and outburst frequency. As demonstrated here, good time coverage of Io's volcanic activity is particularly valuable during missions such as EXCEED and the upcoming Juno arrival, when the data can be tied to other components of the Jovian system.

\section{Acknowledgments}

The authors would like to thank A. Davies for valuable insight at many stages of this work; A. Davies and an anonymous reviewer for careful reading and thoughtful comments; and J. Rathbun for interesting and helpful discussion throughout. In addition, the authors are grateful to A. Stephens and T. Geballe for supporting the Gemini N observing program, and to the Gemini N observers for collecting the data. This research was partially supported by the National Science Foundation grant AST-1313485 to UC Berkeley and by the National Science Foundation Graduate Research Fellowship to K. de Kleer under Grant DGE-1106400. This work made use of the JPL Solar System Dynamics high-precision ephemerides through the HORIZONS system. Data were obtained with the W.M. Keck Observatory, which is operated by the California Institute of Technology, the University of California, and the National Aeronautics and Space Administration. The Observatory was made possible by the generous financial support of the W.M. Keck Foundation. The authors extend special thanks to those of Hawaiian ancestry on whose sacred mountain we are privileged to be guests. Without their generous hospitality, none of the observations presented would have been possible. 


\section{A. An Assessment of Retrieval Uncertainties and Detection Limits}

A robust characterization of the uncertainties on position and intensity retrievals is crucial for hot spot identification and correct interpretation of time variability. We systematically explore the accuracy of our position and flux density retrievals, in order to:

(1) Set lower limits on detectable flux density as a function of filter, telescope, and hot spot position, in order to quantify non-detections and meaningfully interpret the variability in activity at a given hot spot; and

(2) Realistically evaluate the uncertainties on our retrievals for hot spot position and flux density and increase the quoted errorbars if necessary.

Whether or not a hot spot is detectable on a given night is affected by: the PSF, which depends on the filter, telescope, and the atmospheric and seeing conditions; the intrinsic brightness of the hot spot; foreshortening due the hot spot's location relative to the limb; the locations of other hot spots on the disk; and the Earth-Io distance, which changes the effective resolution on Io's disk.

We test the accuracy of our retrieval methods on simulated datasets as follows. We create simulated hot spots by convolving a delta function of the desired flux density with a PSF derived from the standard star for that night. The flux densities are corrected for geometric foreshortening according to the standard cosine law, and added to real observations in a region of the disk relatively free from bright hot spots. We test 15 different flux densities between 1 and $100 \mathrm{GW} / \mu \mathrm{m} / \mathrm{sr}$, inserted at 12 different values of $\mu$ between 0.99 (disk center) and 0.1 (limb). Figure A.12 shows the result of inserting a simulated hot spot of moderate brightness at different disk locations.
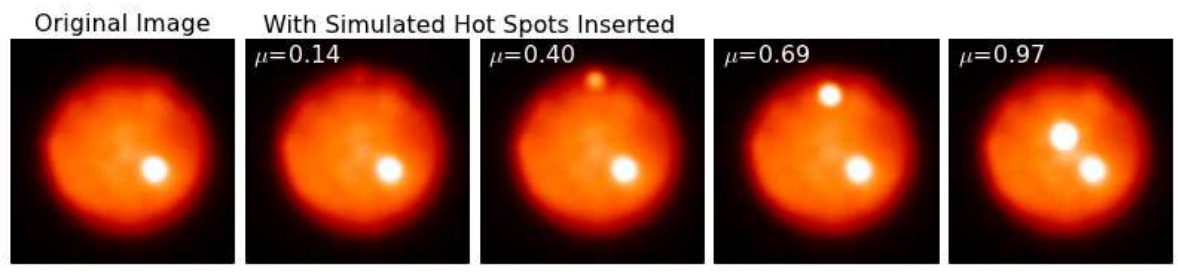

Figure A.12: Simulated hot spots inserted into a Gemini N L'-band observation. The value of $\mu$ for the simulated hot spot is indicated on each plot; all simulated hot spots have an intrinsic flux density of $30 \mathrm{GW} / \mu \mathrm{m} / \mathrm{sr}$ before geometric foreshortening is applied.

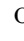

Gemini N. The errors in the retrieved flux densities and positions are shown in Figure A.13 for each choice our analysis (see Section 3.1), including correcting the flux density according to the retrieved emission angle, which may differ from the actual emission angle if the position retrieval is poor. We perform this analysis on base images from multiple different nights, for data in the L' and Ms filters on Keck, and in the L' filter on

After creating the simulated datasets, we retrieve the hot spot flux densities by the same method used in 
of flux density and $\mu$, averaged over all the nights for which we performed this analysis.

As expected, hot spots become increasingly difficult to detect at fainter intrinsic brightnesses, at locations nearer the limb, and in the vicinity of brighter hot spots. For each telescope and filter, we determine and parameterize the approximate flux density $F(\mu)$ where a hot spot becomes undetectable. On a typical night, in a region of Io's disk free of bright hot spots, we can detect a hot spot when the following conditions are met:

- Keck Lp: $F(\mu)>-17.7 \times \mu+10.9 \mathrm{GW} / \mu \mathrm{m} / \mathrm{sr}$

- Keck Ms: $F(\mu)>-15.0 \times \mu+8.1 \mathrm{GW} / \mu \mathrm{m} / \mathrm{sr}$

- Gemini Lp: $F(\mu)>-20.0 \times \mu+13.9 \mathrm{GW} / \mu \mathrm{m} / \mathrm{sr}$

These conditions give an approximate upper limit on the flux density in cases when we observe the location of a known hot spot but do not detect emission. The conditions are shown as dashed lines on Figure A.13, and the detectability regimes are compared in Figure A.14. We find that we can make detections closer to the limb with the Ms filter than the L' filter; the disk is dimmer at longer wavelengths, improving the hot spot contrast. We also have a much greater chance of detecting a hot spot with the Keck telescope over Gemini N. For example, at a disk position of $\mu=0.5$, we can detect hot spots down to $\sim 2 \mathrm{GW} / \mu \mathrm{m} / \mathrm{sr}$ with Keck, while a hot spot needs to be twice as bright to be detected by Gemini N at the same location. In all observations, the detection limit very near the limb $(\mu \leq 0.2)$ is highly sensitive to various factors and cannot be simply quantified; a hot spot at these locations may not be detected even if it meets the above conditions.

In addition to quantifying our limits on hot spot detections, we use this analysis to evaluate uncertainties on our position and intensity retrievals. The deviations between retrieved values and actual values are shown in Figure A.13. We define a region of accurate intensity retrieval where the intensity is retrieved to within $\sim 15 \%$. The boundary of this region is shown as a dashed line in Figure A.13, and compared between telescopes and filters in Figure A.14. For position retrievals, we define three regions for where the error in the retrieved position (in degrees of latitude/longitude) is $>5^{\circ}, \sim 3^{\circ}$, and $\leq 1^{\circ}$. The boundaries between these regions are also shown in Figure A.13, and shown for each telescope and filter in Figure A.15. For very faint hot spots, or very high emission angles, retrievals are additionally uncertain, and are particularly sensitive to observing conditions, phase angle, and nearby hot spots. Flux density uncertainties are in the $50 \%$ range for hot spots detected by Gemini $\mathrm{N}$ with $F_{L^{\prime}}<3 \mathrm{GW} / \mu \mathrm{m} / \mathrm{sr}$, and in the $25 \%$ range for hot spots detected at Keck with $F<1.5 \mathrm{GW} / \mu \mathrm{m} / \mathrm{sr}$ in both filters. In addition, at locations limb-ward of $\mu=0.3$, positions cannot be confidently established to better than $3^{\circ}$. 

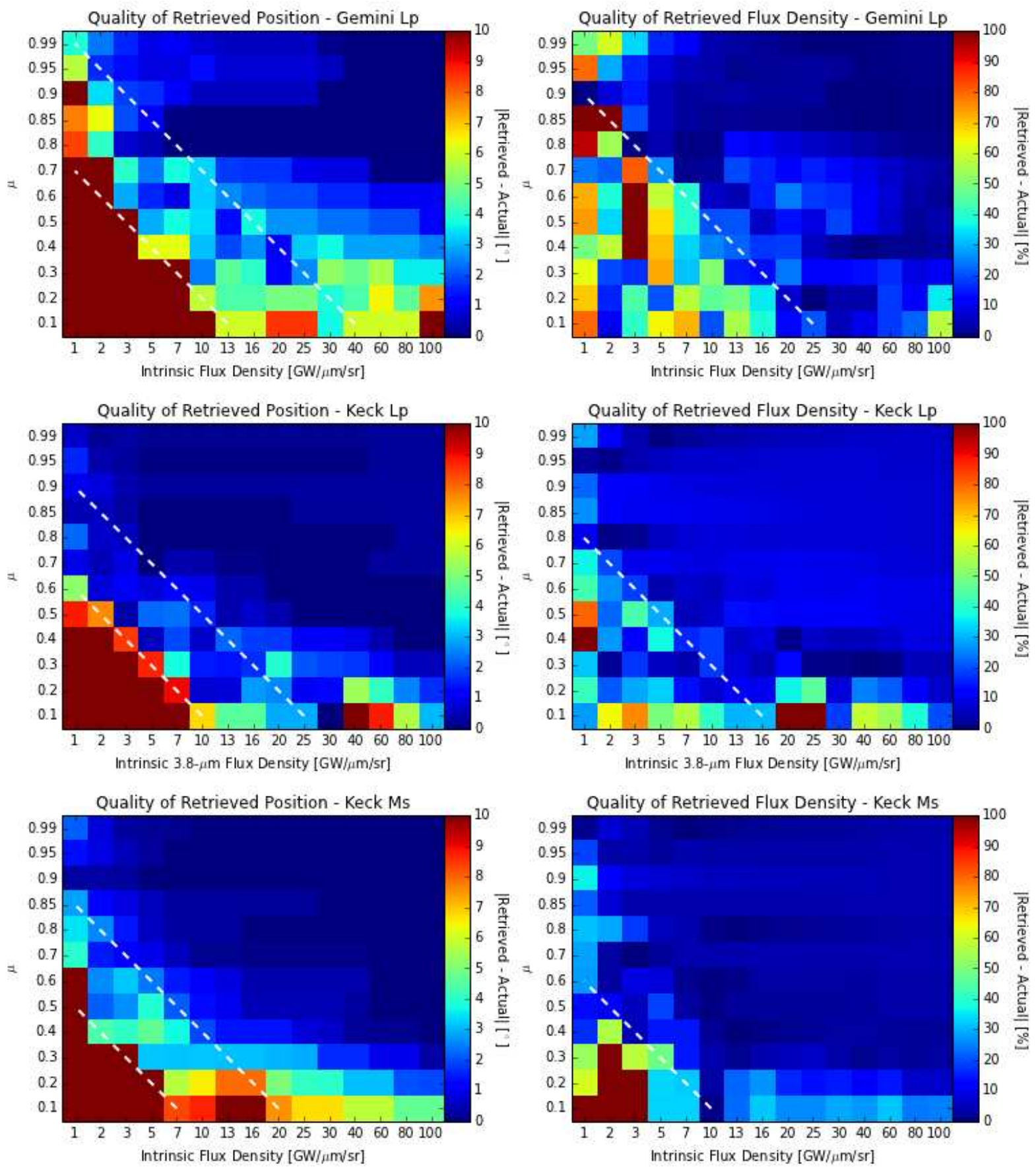

Figure A.13: Error in the retrievals of positions and flux densities of fake hot spots inserted into real Io data, as a function of the cosine of the emission angle $\mu$ and intrinsic flux density (see Section A). The white dashed lines mark the approximate boundaries in this parameter space where the retrievals begin to fail, and are the basis for the detectability criteria and retrieval uncertainty regimes discussed in the text. Errors in position retrievals are given in degrees of latitude/longitude, and errors in flux density retrievals are given in percent of actual flux density.

We apply these results to our analysis as follows:

- We adjust the uncertainties on our retrieved positions so that they are no smaller than $3^{\circ}$ or $5^{\circ}$ depending on which region of Figure A.15 they fall within. We detected no hot spots within the 

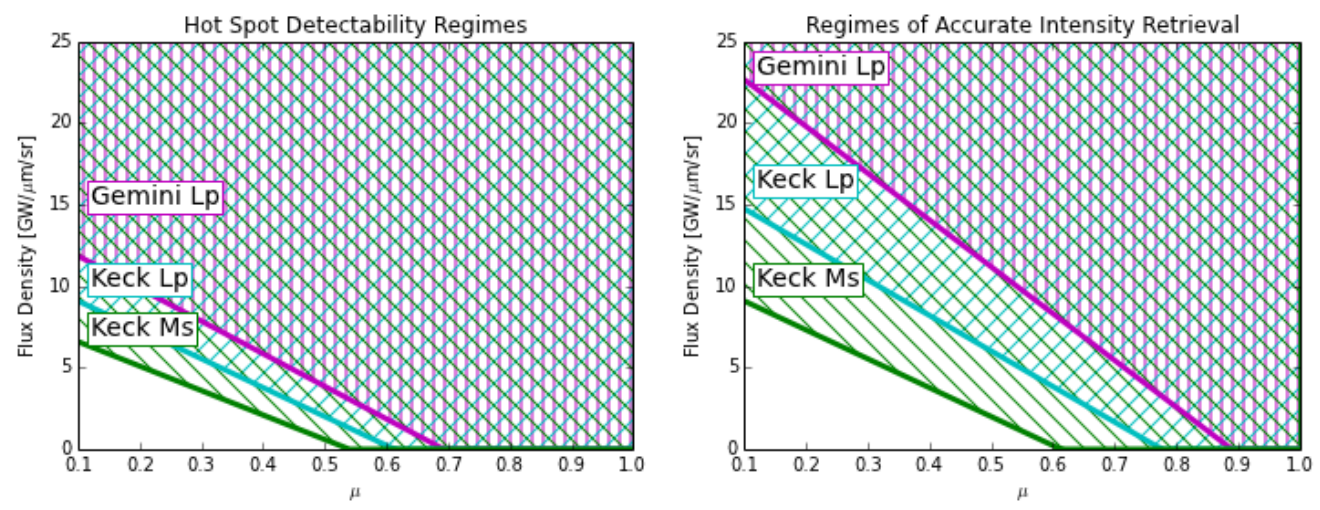

Figure A.14: (Left) Summary of the hot spot detection limits for our observations. The curves indicate the lower limit on the flux density that can be detected at a given disk position; the hatched areas indicate regions where detection is possible. (Right) Summary of flux density-emission angle combinations where intensities can be accurately retrieved. The hatched regions indicate where intensities can be retrieved to $\sim 15 \%$. In both cases these limits may vary from night to night due to observing conditions and the distribution of hot spots on Io's disk.
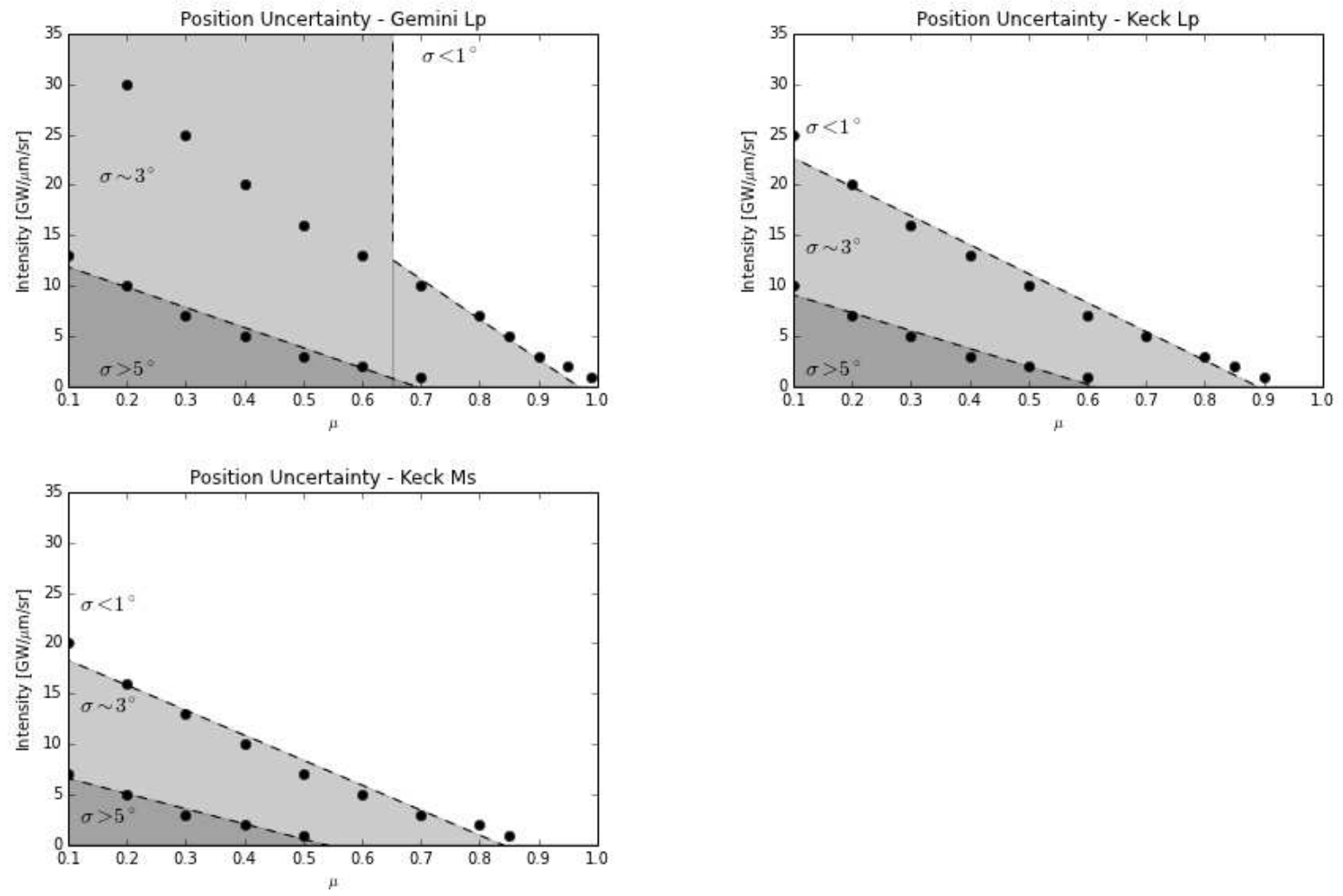

Figure A.15: Comparison of position uncertainty regimes for the Keck L' and Ms filters and the Gemini N L' filter based on simulated hot spot analysis.

undetectable zone (dark gray), but we increased the uncertainties on 30-45\% of hot spots falling within the $\sigma \sim 3^{\circ}$ zone (light gray) in order to satisfy this criterion.

- We adjust the uncertainties on our retrieved flux densities by increasing them to $50 \%$ when the detection 
does not fall into the zone of accurate intensity retrieval. No Ms-band detections fell within this range, but $\sim 5 \%$ of L'-band detections were modified by this criterion.

- For faint hot spots $(<1.5$ or $<3.0 \mathrm{GW} / \mu \mathrm{m} / \mathrm{sr}$ at Keck and Gemini $\mathrm{N}$ respectively), we adjust retrieved flux density uncertainties to be no less than 50\%. This adjustment affected $10-15 \%$ of hot spot detections.

- For hot spots near the limb ( $\mu<0.35$ or $<0.65$ for Keck and Gemini N respectively), we adjust retrieved position uncertainties to be no less than $3^{\circ}$. This adjustment had a minimal effect on retrieved positions, since hot spot detections in these regions were few, and original position uncertainty estimates were already high. 


\section{B. Tables}

Table B.3: Observations

\begin{tabular}{|c|c|c|c|}
\hline UT Date & Telescope/Instrument & $\mathrm{CML}^{a}$ & Filters \\
\hline $2013-08-15$ & Keck/NIRC2 & $337^{\circ}$ & Kc-Ms \\
\hline 2013-08-20 & Keck/NIRC2 & $275^{\circ}$ & Kc-Ms \\
\hline 2013-08-21 & Keck/NIRC2 & $115^{\circ}$ & Kc-Ms \\
\hline 2013-08-22 & Keck/NIRC2 & $319^{\circ}$ & Kc-Ms \\
\hline 2013-08-23 & Keck/NIRC2 & $161^{\circ}$ & Kc-Ms \\
\hline 2013-08-29 & Gemini N/NIRI & $301^{\circ}$ & $\mathrm{L}^{\prime}$ \\
\hline 2013-08-30 & Gemini N/NIRI & $144^{\circ}$ & $L^{\prime}$ \\
\hline 2013-09-01 & Gemini N/NIRI & $191^{\circ}$ & $\mathrm{L}^{\prime}$ \\
\hline 2013-09-02 & Gemini N/NIRI & $34^{\circ}$ & $\mathrm{L}^{\prime}$ \\
\hline 2013-09-03 & Gemini N/NIRI & $237^{\circ}$ & $\mathrm{L}^{\prime}$ \\
\hline 2013-09-04 & Gemini N/NIRI & $81^{\circ}$ & $\mathrm{L}^{\prime}$ \\
\hline 2013-09-05 & Gemini N/NIRI & $284^{\circ}$ & $\mathrm{L}^{\prime}$ \\
\hline 2013-09-06 & Gemini N/NIRI & $127^{\circ}$ & $\mathrm{L}^{\prime}$ \\
\hline 2013-09-07 & Gemini N/NIRI & $331^{\circ}$ & $\mathrm{L}^{\prime}$ \\
\hline 2013-09-09 & Gemini N/NIRI & $17^{\circ}$ & $\mathrm{L}^{\prime}$ \\
\hline 2013-09-10 & Gemini N/NIRI & $221^{\circ}$ & $\mathrm{L}^{\prime}$ \\
\hline 2013-11-18 & Keck/NIRC2 & $215^{\circ}$ & Kc-Ms \\
\hline 2013-11-26 & Gemini N/NIRI & $55^{\circ}$ & $\mathrm{L}^{\prime}$ \\
\hline 2013-11-27 & Gemini N/NIRI & $258^{\circ}$ & $L^{\prime}$ \\
\hline 2013-11-28 & Gemini N/NIRI & $102^{\circ}$ & $L^{\prime}$ \\
\hline 2013-11-29 & Gemini N/NIRI & $305^{\circ}$ & $L^{\prime}$ \\
\hline 2013-12-02 & Gemini N/NIRI & $154^{\circ}$ & $\mathrm{L}^{\prime}$ \\
\hline 2013-12-03 & Gemini N/NIRI & $26^{\circ}$ & $\mathrm{L}^{\prime}$ \\
\hline 2013-12-04 & Gemini N/NIRI & $202^{\circ}$ & $\mathrm{L}^{\prime}$ \\
\hline 2013-12-05 & Gemini N/NIRI & $81^{\circ}$ & $\mathrm{L}^{\prime}$ \\
\hline 2013-12-06 & Gemini N/NIRI & $274^{\circ}$ & $\mathrm{L}^{\prime}$ \\
\hline 2013-12-12 & Gemini N/NIRI & $72^{\circ}$ & $L^{\prime}$ \\
\hline 2013-12-13 & Gemini N/NIRI & $232^{\circ}$ & $L^{\prime}$ \\
\hline 2013-12-14 & Gemini N/NIRI & $119^{\circ}$ & $L^{\prime}$ \\
\hline 2013-12-15 & Gemini N/NIRI & $321^{\circ}$ & $L^{\prime}$ \\
\hline 2014-01-20 & Keck/NIRC2 & $33^{\circ}$ & Kc-Ms \\
\hline 2014-02-08 & Keck/NIRC2 & $274^{\circ}$ & Kc-Ms \\
\hline 2014-02-10 & Keck/NIRC2 & $318^{\circ}$ & Kc-Ms \\
\hline 2014-03-07 & Gemini N/NIRI & $36^{\circ}$ & $\mathrm{Kc}, \mathrm{L}^{\prime}$ \\
\hline 2014-03-10 & Gemini N/NIRI & $259^{\circ}$ & $\mathrm{Kc}, \mathrm{L}^{\prime}$ \\
\hline 2014-03-11 & Keck/NIRC2 & $132^{\circ}$ & Kc-Ms \\
\hline 2014-03-11 & Gemini N/NIRI & $100^{\circ}$ & $\mathrm{Kc}, \mathrm{L}^{\prime}$ \\
\hline 2014-03-12 & Gemini N/NIRI & $303^{\circ}$ & $\mathrm{Kc}, \mathrm{L}^{\prime}$ \\
\hline 2014-03-14 & Gemini N/NIRI & $25^{\circ}$ & $\mathrm{Kc}, \mathrm{L}$ \\
\hline 2014-03-27 & Gemini N/NIRI & $130^{\circ}$ & $\mathrm{Kc}, \mathrm{L}^{\prime}$ \\
\hline 2014-03-28 & Gemini N/NIRI & $338^{\circ}$ & $\mathrm{Kc}, \mathrm{L}^{\prime}$ \\
\hline 2014-04-03 & Gemini N/NIRI & $105^{\circ}$ & $\mathrm{Kc}, \mathrm{L}^{\prime}$ \\
\hline 2014-10-03 & Gemini N/NIRI & $308^{\circ}$ & $\mathrm{Kc}, \mathrm{L}^{\prime}$ \\
\hline 2014-10-09 & Gemini N/NIRI & $87^{\circ}$ & $\mathrm{Kc}, \mathrm{L}^{\prime}$ \\
\hline 2014-10-10 & Gemini N/NIRI & $291^{\circ}$ & $\mathrm{Kc}, \mathrm{L}^{\prime}$ \\
\hline 2014-10-22 & Gemini N/NIRI & $210^{\circ}$ & $\mathrm{Kc}, \mathrm{L}$ \\
\hline 2014-10-23 & Gemini N/NIRI & $56^{\circ}$ & $\mathrm{Kc}, \mathrm{L}$ \\
\hline 2014-10-24 & Gemini N/NIRI & $258^{\circ}$ & $\mathrm{Kc}, \mathrm{L}^{\prime}$ \\
\hline 2014-10-25 & Gemini N/NIRI & $95^{\circ}$ & $\mathrm{Kc}, \mathrm{L}$, \\
\hline 2014-10-27 & Gemini N/NIRI & $146^{\circ}$ & $\mathrm{Kc}, \mathrm{L}^{\prime}$ \\
\hline 2014-10-30 & Keck/NIRC2 & $38^{\circ}$ & Kc-Ms \\
\hline 2014-10-31 & Keck/NIRC2 & $242^{\circ}$ & Kc-Ms \\
\hline
\end{tabular}




\begin{tabular}{|c|c|c|c|}
\hline 2014-11-25 & Gemini N/NIRI & $284^{\circ}$ & $\mathrm{Kc}, \mathrm{L}^{\prime}$ \\
\hline $2014-11-27$ & Gemini N/NIRI & $334^{\circ}$ & $\mathrm{Kc}, \mathrm{L}^{\prime}$ \\
\hline 2014-11-28 & Gemini N/NIRI & $153^{\circ}$ & $\mathrm{Kc}, \mathrm{L}^{\prime}$ \\
\hline 2014-11-29 & Gemini N/NIRI & $22^{\circ}$ & $\mathrm{Kc}, \mathrm{L}^{\prime}$ \\
\hline 2014-11-30 & Gemini N/NIRI & $228^{\circ}$ & $\mathrm{Kc}, \mathrm{L}$ \\
\hline 2014-12-01 & Gemini N/NIRI & $71^{\circ}$ & $\mathrm{Kc}, \mathrm{L}^{\prime}$ \\
\hline 2014-12-02 & Keck/NIRC2 & $275^{\circ}$ & Kc-Ms \\
\hline $2014-12-06$ & Gemini N/NIRI & $329^{\circ}$ & $\mathrm{Kc}, \mathrm{L}^{\prime}$ \\
\hline 2014-12-08 & Gemini N/NIRI & $50^{\circ}$ & $\mathrm{Kc}, \mathrm{L}^{\prime}$ \\
\hline 2014-12-09 & Gemini N/NIRI & $259^{\circ}$ & $\mathrm{Kc}, \mathrm{L}^{\prime}$ \\
\hline $2014-12-10$ & Gemini N/NIRI & $98^{\circ}$ & $\mathrm{Kc}, \mathrm{L}^{\prime}$ \\
\hline 2014-12-15 & Gemini N/NIRI & $40^{\circ}$ & $\mathrm{Kc}, \mathrm{L}$ \\
\hline 2014-12-16 & Gemini N/NIRI & $247^{\circ}$ & $\mathrm{Kc}, \mathrm{L}^{\prime}$ \\
\hline $2014-12-18$ & Gemini N/NIRI & $293^{\circ}$ & $\mathrm{Kc}, \mathrm{L}^{\prime}$ \\
\hline 2015-01-10 & Gemini N/NIRI & $296^{\circ}$ & $\mathrm{Kc}, \mathrm{L}$ \\
\hline 2015-01-11 & Keck/NIRC2 & $138^{\circ}$ & $\mathrm{Kc}-\mathrm{Ms}$ \\
\hline 2015-01-12 & Keck/NIRC2 & $338^{\circ}$ & Kc-Ms \\
\hline 2015-01-13 & Gemini N/NIRI & $134^{\circ}$ & $\mathrm{Kc}, \mathrm{L}^{\prime}$ \\
\hline 2015-01-14 & Gemini N/NIRI & $328^{\circ}$ & $\mathrm{Kc}, \mathrm{L}$ \\
\hline 2015-01-15 & Gemini N/NIRI & $231^{\circ}$ & $\mathrm{Kc}, \mathrm{L}^{\prime}$ \\
\hline 2015-01-16 & Keck/NIRC2 & $74^{\circ}$ & Kc-Ms \\
\hline $2015-01-22$ & Gemini N/NIRI & $154^{\circ}$ & $\mathrm{Kc}, \mathrm{L}^{\prime}$ \\
\hline $2015-01-26$ & Gemini N/NIRI & $250^{\circ}$ & $\mathrm{Kc}, \mathrm{L}^{\prime}$ \\
\hline $2015-03-25$ & Gemini N/NIRI & $149^{\circ}$ & $\mathrm{Kc}, \mathrm{L}^{\prime}$ \\
\hline $2015-03-26$ & Gemini N/NIRI & $29^{\circ}$ & $\mathrm{Kc}, \mathrm{L}^{\prime}$ \\
\hline 2015-03-27 & Gemini N/NIRI & $213^{\circ}$ & $\mathrm{Kc}, \mathrm{L}^{\prime}$ \\
\hline $2015-03-28$ & Gemini N/NIRI & $42^{\circ}$ & $\mathrm{Kc}, \mathrm{L}$ \\
\hline 2015-03-29 & Gemini N/NIRI & $244^{\circ}$ & $\mathrm{Kc}, \mathrm{L}^{\prime}$ \\
\hline 2015-03-31 & Keck/NIRC2 & $292^{\circ}$ & Kc-Ms \\
\hline 2015-04-01 & Keck/NIRC2 & $134^{\circ}$ & $\mathrm{Kc}-\mathrm{Ms}$ \\
\hline 2015-04-02 & Keck/NIRC2 & $338^{\circ}$ & Kc-Ms \\
\hline 2015-04-04 & Keck/NIRC2 & $28^{\circ}$ & Kc-Ms \\
\hline 2015-04-05 & Gemini N/NIRI & $231^{\circ}$ & $\mathrm{Kc}, \mathrm{L}^{\prime}$ \\
\hline 2015-04-06 & Gemini N/NIRI & $70^{\circ}$ & $\mathrm{Kc}, \mathrm{L}^{\prime}$ \\
\hline 2015-04-09 & Gemini N/NIRI & $322^{\circ}$ & $\mathrm{Kc}, \mathrm{L}^{\prime}$ \\
\hline 2015-04-17 & Gemini N/NIRI & $149^{\circ}$ & $\mathrm{Kc}, \mathrm{L}^{\prime}$ \\
\hline 2015-04-19 & Gemini N/NIRI & $197^{\circ}$ & $\mathrm{Kc}, \mathrm{L}^{\prime}$ \\
\hline 2015-04-20 & Gemini N/NIRI & $40^{\circ}$ & $\mathrm{Kc}, \mathrm{L}^{\prime}$ \\
\hline 2015-04-21 & Gemini N/NIRI & $244^{\circ}$ & $\mathrm{Kc}, \mathrm{L}^{\prime}$ \\
\hline 2015-04-22 & Gemini N/NIRI & $89^{\circ}$ & $\mathrm{Kc}, \mathrm{L}^{\prime}$ \\
\hline 2015-04-26 & Gemini N/NIRI & $206^{\circ}$ & $\mathrm{Kc}, \mathrm{L}^{\prime}$ \\
\hline 2015-04-27 & Gemini N/NIRI & $24^{\circ}$ & Kc,L' \\
\hline 2015-04-29 & Keck/NIRC2 & $73^{\circ}$ & Kc-Ms \\
\hline 2015-05-05 & Keck/NIRC2 & $213^{\circ}$ & Kc-Ms \\
\hline 2015-06-05 & Keck/NIRC2 & $38^{\circ}$ & $\mathrm{Kc}-\mathrm{Ms}$ \\
\hline $2015-11-23$ & Keck/NIRC2 & $212^{\circ}$ & Kc-Ms \\
\hline $2015-12-22$ & Keck/NIRC2 & $327^{\circ}$ & Kc-Ms \\
\hline $2015-12-25$ & Keck/NIRC2 & $106^{\circ}$ & Kc-Ms \\
\hline
\end{tabular}

${ }^{a} \mathrm{CML}=$ Central Meridian Longitude of Io

Table B.4: Hot Spot Detections

\begin{tabular}{llllllll}
\hline ID & Date & $\begin{array}{l}\text { Latitude } \\
{\left[{ }^{\circ} \mathrm{N}\right]}\end{array}$ & $\begin{array}{l}\text { Longitude } \\
{\left[{ }^{\circ} \mathrm{W}\right]}\end{array}$ & $\mu$ & $\mathrm{F}_{K c}$ & $\begin{array}{c}\mathrm{F}_{H 2 O} 2 O \\
{[\text { all intensities in } \mathrm{GW} / \mu \mathrm{m} / \mathrm{sr}]}\end{array}$ & $\begin{array}{c}\mathrm{F}_{M s} \\
\mathrm{~F} L^{\prime}\end{array}$ \\
\hline $\mathrm{A}$ & $12-03-13$ & $-33.8 \pm 2.0$ & $20.1 \pm 2.1$ & 0.83 & & $\begin{array}{l}\mathrm{T} \\
{[\mathrm{K}]}\end{array}$ & $\begin{array}{l}\mathrm{P} \\
{[\mathrm{GW}]}\end{array}$ \\
\hline
\end{tabular}




\begin{tabular}{|c|c|c|c|c|c|c|c|c|c|c|}
\hline & $01-20-14$ & $-34.9 \pm 0.8$ & $20.9 \pm 0.8$ & 0.79 & & $1.6 \pm 0.2$ & $3.1 \pm 0.5$ & $5.4 \pm 0.8$ & $441_{-31}^{+34}$ & $230_{-70}^{+90}$ \\
\hline & 02-10-14 & $-34.1 \pm 0.5$ & $23.4 \pm 2.1$ & 0.35 & & & $5.1 \pm 2.6$ & $4.6 \pm 0.9$ & $590_{-170}^{+230}$ & $110_{-60}^{+120}$ \\
\hline & $03-07-14$ & $-34.9 \pm 2.0$ & $25.9 \pm 2.2$ & 0.81 & & & $2.2 \pm 1.1$ & & & \\
\hline & $03-14-14$ & $-34.7 \pm 2.0$ & $24.8 \pm 2.1$ & 0.82 & & & $3.3 \pm 0.5$ & & & \\
\hline & $10-30-14$ & $-33.7 \pm 0.8$ & $21.2 \pm 0.8$ & 0.79 & & & $3.0 \pm 0.4$ & $5.8 \pm 0.9$ & $430_{-60}^{+70}$ & $260_{-110}^{+190}$ \\
\hline & $11-29-14$ & $-35.5 \pm 2.1$ & $20.6 \pm 2.1$ & 0.81 & & & $3.2 \pm 0.6$ & & & \\
\hline & $12-08-14$ & $-34.1 \pm 1.6$ & $19.1 \pm 2.5$ & 0.71 & & & $3.4 \pm 1.7$ & & & \\
\hline & $12-15-14$ & $-34.2 \pm 1.8$ & $19.6 \pm 2.3$ & 0.77 & & & $2.9 \pm 1.4$ & & & \\
\hline & $01-12-15$ & $-35.4 \pm 1.0$ & $22.6 \pm 1.8$ & 0.59 & & & $3.2 \pm 1.6$ & $4.9 \pm 0.7$ & $470_{-120}^{+160}$ & $170_{-100}^{+240}$ \\
\hline & $01-16-15$ & $-34.1 \pm 1.0$ & $21.0 \pm 1.8$ & 0.49 & & & $3.4 \pm 1.7$ & $4.6 \pm 0.7$ & $520_{-140}^{+190}$ & $130_{-70}^{+160}$ \\
\hline & $03-26-15$ & $-37.0 \pm 2.0$ & $22.1 \pm 2.2$ & 0.79 & & & $3.4 \pm 0.5$ & & & \\
\hline & $03-28-15$ & $-34.6 \pm 1.8$ & $25.3 \pm 2.3$ & 0.79 & & & $3.2 \pm 0.5$ & & & \\
\hline & $04-02-15$ & $-33.5 \pm 0.6$ & $23.9 \pm 1.3$ & 0.59 & & & $3.5 \pm 0.8$ & $4.7 \pm 0.7$ & $540_{-110}^{+140}$ & $121_{-47}^{+78}$ \\
\hline & $04-04-15$ & $-33.9 \pm 0.5$ & $24.8 \pm 0.5$ & 0.83 & & & $3.9 \pm 0.6$ & $6.1 \pm 0.9$ & $\begin{array}{l}490_{-70}^{+80} \\
\end{array}$ & $190_{-70}^{+110}$ \\
\hline & $04-20-15$ & $-34.6 \pm 1.9$ & $27.2 \pm 2.2$ & 0.80 & & & $3.7 \pm 0.6$ & & & \\
\hline & $04-27-15$ & $-35.8 \pm 2.0$ & $24.4 \pm 2.1$ & 0.81 & & & $4.0 \pm 0.6$ & & & \\
\hline & $04-29-15$ & $-34.7 \pm 0.7$ & $23.4 \pm 1.2$ & 0.53 & & & $4.1 \pm 0.9$ & $5.4 \pm 0.8$ & $550_{-100}^{+120}$ & $139_{-50}^{+77}$ \\
\hline & $06-05-15$ & $-32.2 \pm 0.7$ & $23.5 \pm 0.9$ & 0.81 & & $3.3 \pm 0.5$ & $4.8 \pm 0.7$ & $7.1 \pm 1.1$ & $514_{-45}^{+49}$ & $210_{-50}^{+70}$ \\
\hline & $11-23-15$ & $-35.5 \pm 0.7$ & $21.3 \pm 1.9$ & 0.48 & & & $3.3 \pm 1.7$ & $5.2 \pm 0.8$ & $490_{-130}^{+170}$ & $160_{-90}^{+220}$ \\
\hline & $12-25-15$ & $-34.6 \pm 0.6$ & $22.7 \pm 1.9$ & 0.48 & & & $4.0 \pm 1.4$ & $6.1 \pm 0.9$ & $470_{-100}^{+130}$ & $210_{-110}^{+240}$ \\
\hline & Average & $-34.4 \pm 0.9$ & $22.8 \pm 2.1$ & & & & & & & \\
\hline Patera & & -36 & 22 & & & & & & & \\
\hline $\mathrm{B}$ & $01-20-14$ & $-17.3 \pm 0.7$ & $32.3 \pm 1.1$ & 0.95 & & & $1.5 \pm 0.4$ & $3.3 \pm 0.5$ & $400_{-60}^{+80}$ & $190_{-100}^{+210}$ \\
\hline & $10-30-14$ & $-19.1 \pm 1.2$ & $30.9 \pm 0.7$ & 0.94 & & & $1.0 \pm 0.4$ & $1.5 \pm 0.2$ & $490_{-120}^{+160}$ & $49_{-28}^{+\overline{6} 6}$ \\
\hline & $01-16-15$ & $-16.6 \pm 1.8$ & $31.3 \pm 2.3$ & 0.70 & & & & $1.4 \pm 0.7$ & & \\
\hline & $04-02-15$ & $-15.7 \pm 0.8$ & $34.4 \pm 1.9$ & 0.55 & & & & $1.4 \pm 0.5$ & & \\
\hline & $04-04-15$ & $-17.6 \pm 0.9$ & $35.2 \pm 0.5$ & 0.94 & & & $1.0 \pm 0.5$ & $2.0 \pm 0.3$ & $420_{-100}^{+140}$ & $100_{-60}^{+160}$ \\
\hline & 04-29-15 & $-15.2 \pm 1.0$ & $36.5 \pm 1.2$ & 0.77 & & & & $1.4 \pm 0.5$ & & \\
\hline & 06-05-15 & $-16.1 \pm 1.5$ & $34.5 \pm 0.6$ & 0.96 & & & & $1.3 \pm 0.4$ & & \\
\hline & Average & $-16.8 \pm 1.2$ & $33.8 \pm 1.9$ & & & & & & & \\
\hline $\begin{array}{c}\text { Kanehekili } \\
\text { Fluctus }\end{array}$ & & -15 to -21 & 32 to 36 & & & & & & & \\
\hline $\mathrm{C}$ & $09-02-13$ & $-4.5 \pm 1.4$ & $37.0 \pm 1.4$ & 1.00 & & & $5.0 \pm 0.7$ & & & \\
\hline & 09-04-13 & $-4.4 \pm 1.7$ & $36.2 \pm 2.4$ & 0.73 & & & $4.6 \pm 0.8$ & & & \\
\hline & $09-07-13$ & $-4.6 \pm 1.4$ & $37.3 \pm 3.5$ & 0.46 & & & $10.8 \pm 2.3$ & & & \\
\hline & 09-09-13 & $-5.0 \pm 1.3$ & $36.5 \pm 1.4$ & 0.97 & & & $5.2 \pm 0.8$ & & & \\
\hline & $11-26-13$ & $-4.8 \pm 1.1$ & $37.9 \pm 1.1$ & 0.96 & & & $3.5 \pm 0.5$ & & & \\
\hline & $12-03-13$ & $-4.0 \pm 1.0$ & $37.7 \pm 1.1$ & 0.97 & & & $6.1 \pm 1.1$ & & & \\
\hline & $12-05-13$ & $-4.4 \pm 1.7$ & $38.0 \pm 2.4$ & 0.73 & & & $2.5 \pm 1.2$ & & & \\
\hline & $12-12-13$ & $-5.3 \pm 1.8$ & $34.1 \pm 2.3$ & 0.78 & & & $3.9 \pm 0.6$ & & & \\
\hline & $01-20-14$ & $-4.8 \pm 0.5$ & $38.0 \pm 0.7$ & 0.99 & $3.5 \pm 0.5$ & $4.2 \pm 0.6$ & $4.4 \pm 0.7$ & $4.7 \pm 0.7$ & $690 \pm 60$ & $95_{-14}^{+16}$ \\
\hline & $03-07-14$ & $-3.7 \pm 1.1$ & $39.7 \pm 1.1$ & 1.00 & & & $3.0 \pm 0.5$ & & & \\
\hline & $03-14-14$ & $-3.9 \pm 1.2$ & $39.0 \pm 1.2$ & 0.96 & & & $3.6 \pm 0.5$ & & & \\
\hline & $10-09-14$ & $-3.8 \pm 1.6$ & $39.6 \pm 2.4$ & 0.68 & & & $5.3 \pm 1.0$ & & & \\
\hline & $10-23-14$ & $-3.2 \pm 1.3$ & $40.5 \pm 1.4$ & 0.96 & & & $4.1 \pm 0.6$ & & & \\
\hline & $10-30-14$ & $-4.8 \pm 0.4$ & $36.6 \pm 0.4$ & 1.00 & & & $5.1 \pm 0.8$ & $4.8 \pm 0.7$ & $700_{-110}^{+130}$ & $99_{-17}^{+20}$ \\
\hline & $11-27-14$ & $-4.3 \pm 1.2$ & $39.2 \pm 3.4$ & 0.42 & & & $8.4 \pm 4.2$ & & & \\
\hline & $11-29-14$ & $-4.3 \pm 1.2$ & $37.4 \pm 1.3$ & 0.96 & & & $4.6 \pm 0.7$ & & & \\
\hline & $12-01-14$ & $-4.8 \pm 1.8$ & $37.8 \pm 2.3$ & 0.83 & & & $3.7 \pm 0.7$ & & & \\
\hline & $12-08-14$ & $-5.0 \pm 1.2$ & $35.6 \pm 1.2$ & 0.96 & & & $5.1 \pm 0.8$ & & & \\
\hline & $12-15-14$ & $-5.4 \pm 1.1$ & $36.9 \pm 1.1$ & 0.99 & & & $4.7 \pm 0.7$ & & & \\
\hline & $01-12-15$ & $-4.8 \pm 0.6$ & $34.6 \pm 1.5$ & 0.55 & & $5.0 \pm 0.7$ & $6.0 \pm 0.9$ & $5.7 \pm 0.9$ & $680_{-70}^{+80}$ & $120_{-18}^{+21}$ \\
\hline & $01-16-15$ & $-4.5 \pm 0.4$ & $37.0 \pm 0.8$ & 0.79 & & & $3.9 \pm 0.6$ & $4.5 \pm 0.7$ & $610_{-100}^{+120}$ & $100_{-25}^{+33}$ \\
\hline & $03-26-15$ & $-3.7 \pm 1.1$ & $38.5 \pm 1.2$ & 0.98 & & & $4.5 \pm 0.7$ & & & \\
\hline & $03-28-15$ & $-4.9 \pm 1.1$ & $41.3 \pm 1.1$ & 1.00 & & & $3.9 \pm 0.6$ & & & \\
\hline
\end{tabular}




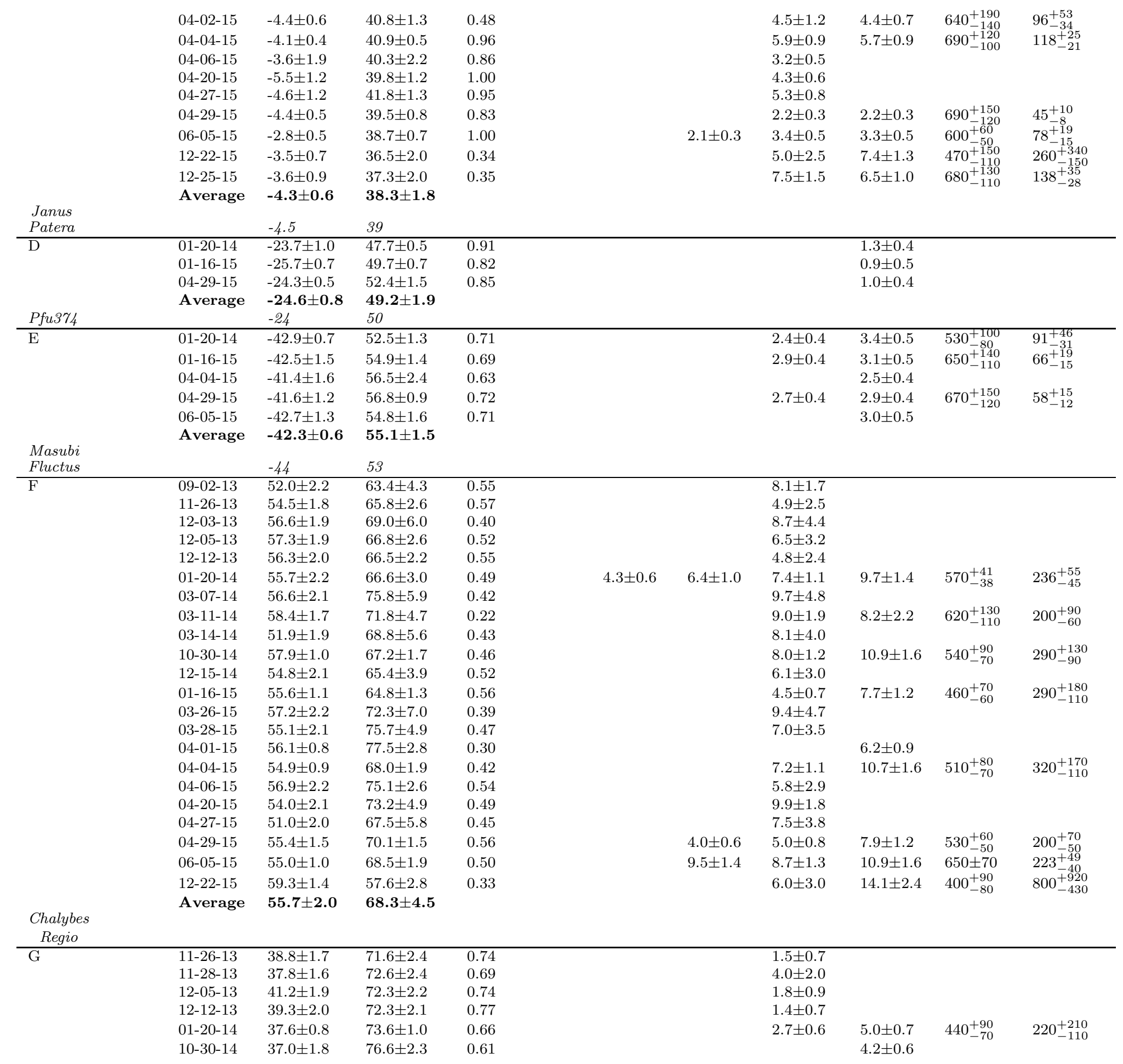




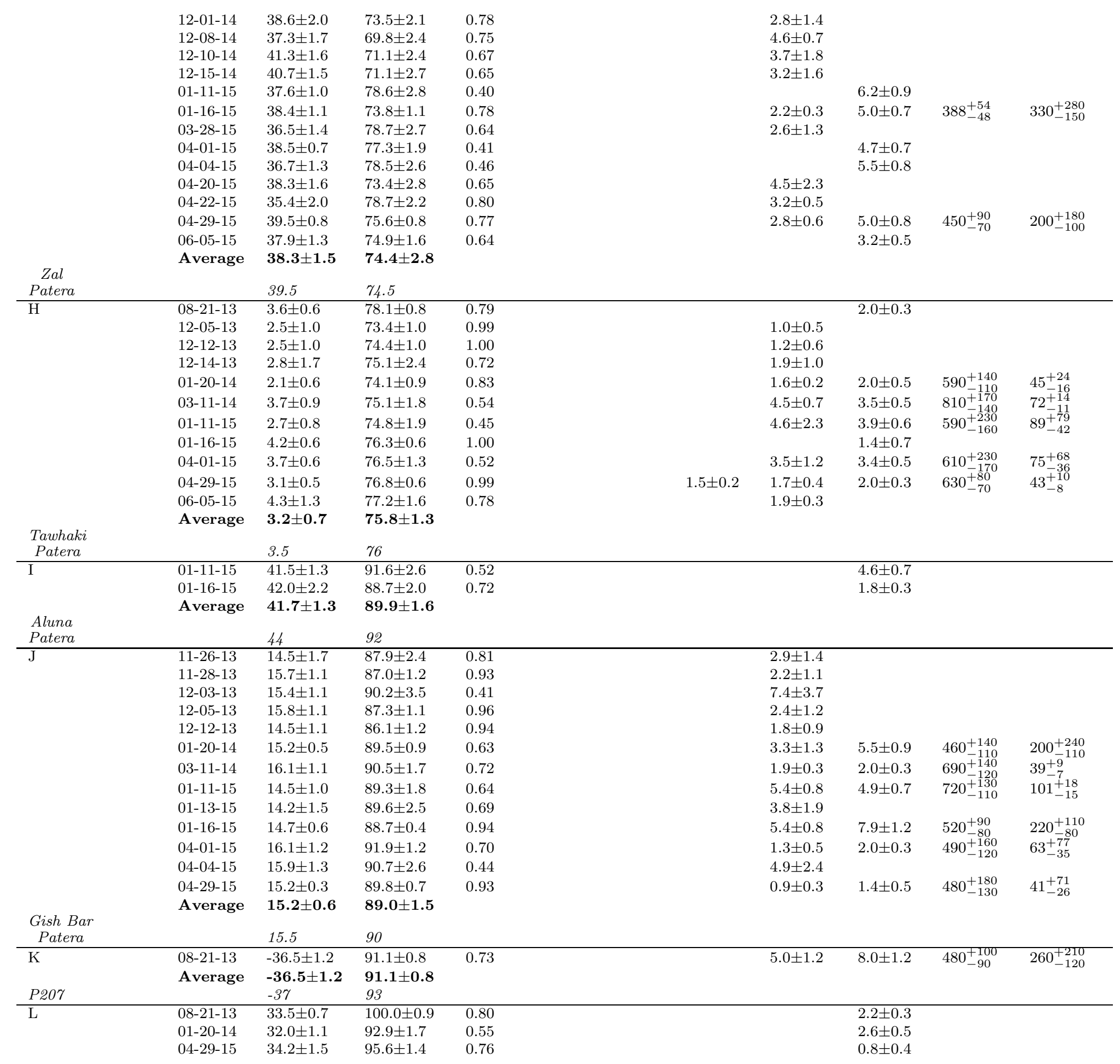




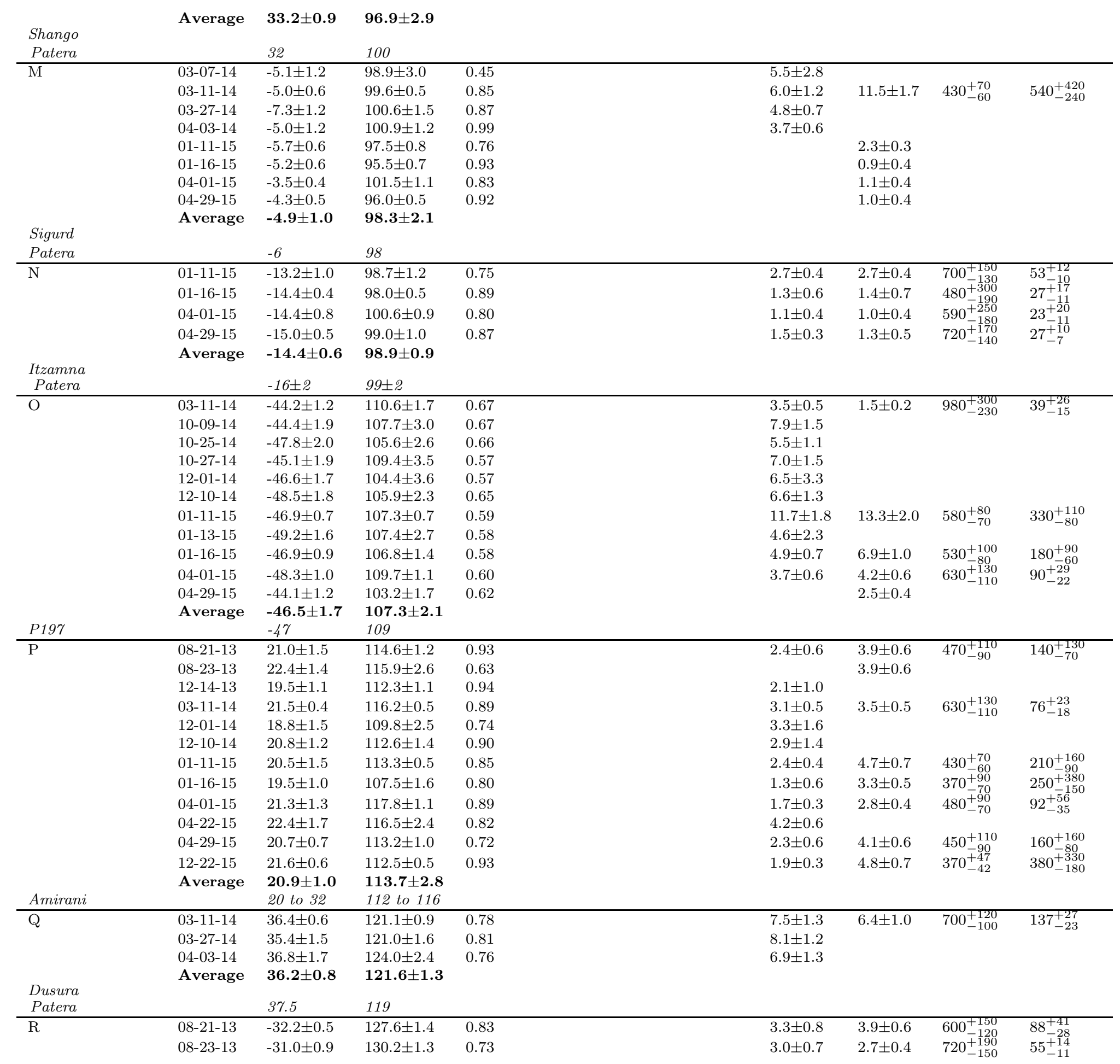




\begin{tabular}{|c|c|c|c|c|c|c|c|c|c|}
\hline & $03-11-14$ & $-33.6 \pm 0.6$ & $129.9 \pm 0.6$ & 0.83 & & & $1.3 \pm 0.6$ & & \\
\hline & $01-11-15$ & $-32.9 \pm 1.6$ & $127.3 \pm 1.1$ & 0.82 & & $1.7 \pm 0.3$ & $1.7 \pm 0.3$ & $720_{-120}^{+140}$ & $33_{-6}^{+7}$ \\
\hline & $01-16-15$ & $-33.5 \pm 0.9$ & $125.9 \pm 1.8$ & 0.52 & & $6.9 \pm 1.0$ & $6.6 \pm 1.0$ & $670_{-100}^{+110}$ & $140_{-25}^{+30}$ \\
\hline & $01-22-15$ & $-34.7 \pm 1.7$ & $129.6 \pm 2.4$ & 0.75 & & $2.4 \pm 1.2$ & & & \\
\hline & $04-01-15$ & $-32.4 \pm 1.0$ & $132.0 \pm 0.8$ & 0.84 & & $1.5 \pm 0.3$ & $1.9 \pm 0.3$ & $560_{-110}^{+130}$ & $47_{-16}^{+24}$ \\
\hline & 04-29-15 & $-35.1 \pm 1.2$ & $130.3 \pm 2.7$ & 0.49 & & & $3.2 \pm 0.5$ & & \\
\hline \multirow{2}{*}{\multicolumn{10}{|c|}{$129.4 \pm 1.8$}} \\
\hline & & & & & & & & & \\
\hline Patera & & -34 & 129 & & & & & & \\
\hline \multirow[t]{3}{*}{$\mathrm{S}$} & $01-11-15$ & $41.6 \pm 2.0$ & $133.0 \pm 2.1$ & 0.74 & & & $1.5 \pm 0.7$ & & \\
\hline & 04-01-15 & $39.5 \pm 1.5$ & $136.4 \pm 1.4$ & 0.77 & & & $1.2 \pm 0.4$ & & \\
\hline & Average & $40.4 \pm 1.2$ & $135.0 \pm 1.6$ & & & & & & \\
\hline Thor & & 38 & 132 & & & & & & \\
\hline \multirow[t]{4}{*}{$\mathrm{T}$} & $01-11-15$ & $-42.0 \pm 1.2$ & $138.6 \pm 1.4$ & 0.74 & & $2.9 \pm 0.4$ & $2.6 \pm 0.4$ & $770_{-140}^{+160}$ & $51_{-6}^{+7}$ \\
\hline & 04-01-15 & $-41.2 \pm 0.7$ & $143.4 \pm 0.9$ & 0.74 & $2.5 \pm 0.4$ & $2.7 \pm 0.4$ & $2.7 \pm 0.4$ & $720_{-80}^{+100}$ & $53_{-7}^{+9}$ \\
\hline & $12-22-15$ & $-41.8 \pm 1.1$ & $135.4 \pm 1.3$ & 0.65 & & $4.6 \pm 0.7$ & $7.8 \pm 1.2$ & $470_{-60}^{+70}$ & $280_{-110}^{+180}$ \\
\hline & Average & $-41.6 \pm 0.6$ & $139.8 \pm 3.2$ & & & & & & \\
\hline P123 & & -42 & 138 & & & & & & \\
\hline \multirow[t]{4}{*}{$\mathrm{U}$} & $01-11-15$ & $-18.2 \pm 0.8$ & $139.2 \pm 0.7$ & 0.95 & & $1.3 \pm 0.6$ & $1.6 \pm 0.2$ & $540_{-150}^{+220}$ & $43_{-24}^{+55}$ \\
\hline & 04-01-15 & $-17.7 \pm 1.0$ & $143.7 \pm 1.0$ & 0.94 & & $0.8 \pm 0.3$ & $1.3 \pm 0.4$ & $510_{-130}^{+180}$ & $34_{-20}^{+51}$ \\
\hline & $12-22-15$ & $-17.4 \pm 1.0$ & $136.9 \pm 1.2$ & 0.82 & & $1.7 \pm 0.2$ & $3.5 \pm 0.5$ & $409_{-50}^{+57}$ & $190_{-80}^{+140}$ \\
\hline & Average & $-17.8 \pm 0.5$ & $140.1 \pm 2.8$ & & & & & & \\
\hline \multicolumn{10}{|l|}{ Tupan } \\
\hline Patera & & -19 & 140 & & & & & & \\
\hline \multirow[t]{5}{*}{$\mathrm{V}$} & $03-25-15$ & $21.5 \pm 1.2$ & $148.7 \pm 1.2$ & 0.93 & & $2.7 \pm 1.3$ & & & \\
\hline & $04-01-15$ & $21.9 \pm 0.9$ & $148.8 \pm 0.7$ & 0.90 & & $3.6 \pm 0.5$ & $6.6 \pm 1.0$ & $440_{-60}^{+70}$ & $270_{-110}^{+180}$ \\
\hline & $04-17-15$ & $21.0 \pm 1.3$ & $150.0 \pm 1.3$ & 0.93 & & $2.0 \pm 1.0$ & & & \\
\hline & $05-05-15$ & $20.6 \pm 1.0$ & $151.1 \pm 2.8$ & 0.47 & & & $5.0 \pm 0.8$ & & \\
\hline & Average & $21.3 \pm 0.5$ & $149.3 \pm 0.9$ & & & & & & \\
\hline \multicolumn{10}{|l|}{ Surya } \\
\hline Patera & & 22 & 151.5 & & & & & & \\
\hline \multirow[t]{14}{*}{$\mathrm{W}$} & $08-21-13$ & $-0.0 \pm 0.6$ & $150.3 \pm 0.5$ & 0.82 & & $2.4 \pm 0.6$ & $3.5 \pm 0.5$ & $530_{-110}^{+140}$ & $93_{-38}^{+65}$ \\
\hline & $08-23-13$ & $0.0 \pm 0.9$ & $152.9 \pm 0.4$ & 0.99 & & $2.7 \pm 0.6$ & $3.8 \pm 0.6$ & $530_{-100}^{+120}$ & $104^{+71}$ \\
\hline & $11-18-13$ & $-1.5 \pm 1.2$ & $154.2 \pm 2.7$ & 0.46 & & $5.3 \pm 0.8$ & & & \\
\hline & $12-02-13$ & $-1.1 \pm 1.0$ & $152.8 \pm 1.0$ & 1.00 & & $2.4 \pm 1.2$ & & & \\
\hline & $12-04-13$ & $-1.2 \pm 1.7$ & $156.5 \pm 2.4$ & 0.70 & & $2.2 \pm 1.1$ & & & \\
\hline & $12-14-13$ & $-2.2 \pm 1.8$ & $154.3 \pm 2.3$ & 0.81 & & $1.9 \pm 0.9$ & & & \\
\hline & 03-11-14 & $-1.3 \pm 0.4$ & $155.2 \pm 1.3$ & 0.92 & & $3.4 \pm 0.5$ & $3.9 \pm 0.6$ & $630_{-110}^{+130}$ & $83_{-20}^{+26}$ \\
\hline & $03-27-14$ & $-1.8 \pm 1.2$ & $156.8 \pm 1.4$ & 0.89 & & $4.0 \pm 0.6$ & & & \\
\hline & $01-11-15$ & $-2.3 \pm 0.7$ & $152.4 \pm 0.4$ & 0.97 & & $2.8 \pm 0.4$ & $3.9 \pm 0.6$ & $530_{-80}^{+100}$ & $104_{-34}^{+51}$ \\
\hline & $04-01-15$ & $-1.1 \pm 0.5$ & $155.9 \pm 0.5$ & 0.93 & & $1.5 \pm 0.2$ & $2.2 \pm 0.3$ & $540_{-80}^{+100}$ & $57_{-19}^{+27}$ \\
\hline & $04-26-15$ & $-1.5 \pm 1.6$ & $156.4 \pm 2.5$ & 0.64 & & $4.3 \pm 2.2$ & & & \\
\hline & $05-05-15$ & $-0.0 \pm 0.6$ & $155.8 \pm 1.3$ & 0.54 & & $4.1 \pm 0.6$ & $4.4 \pm 0.7$ & $650_{-110}^{+130}$ & $94_{-21}^{+26}$ \\
\hline & $12-22-15$ & $-0.8 \pm 1.3$ & $151.3 \pm 1.3$ & 0.71 & & $1.6 \pm 0.2$ & $3.7 \pm 0.6$ & $379_{-45}^{+51}$ & $270_{-120}^{+220}$ \\
\hline & Average & $-1.0 \pm 0.7$ & $153.6 \pm 2.0$ & & & & & & \\
\hline Prometheus & & -1.5 & 155 & & & & & & \\
\hline \multirow[t]{6}{*}{$\mathrm{X}$} & $08-23-13$ & $-16.5 \pm 0.4$ & $161.6 \pm 0.9$ & 0.96 & & $1.4 \pm 0.7$ & $2.1 \pm 0.3$ & $490_{-140}^{+190}$ & $68_{-41}^{+107}$ \\
\hline & $03-11-14$ & $-16.0 \pm 0.4$ & $162.0 \pm 0.5$ & 0.83 & & $2.2 \pm 0.3$ & $1.9 \pm 0.3$ & $770_{-120}^{+150}$ & $38.6_{-4.3}^{+4.8}$ \\
\hline & $01-11-15$ & $-17.2 \pm 0.4$ & $159.8 \pm 0.5$ & 0.89 & & $1.9 \pm 0.3$ & $2.3 \pm 0.3$ & $600_{-100}^{+120}$ & $52_{-15}^{+20}$ \\
\hline & $04-01-15$ & $-16.0 \pm 0.5$ & $164.6 \pm 1.1$ & 0.84 & & $1.1 \pm 0.4$ & $1.7 \pm 0.2$ & $490_{-120}^{+150}$ & $53_{-29}^{+63}$ \\
\hline & $05-05-15$ & $-14.7 \pm 1.0$ & $169.4 \pm 1.8$ & 0.69 & & & $2.1 \pm 0.3$ & & \\
\hline & Average & $-16.2 \pm 0.8$ & $162.3 \pm 3.3$ & & & & & & \\
\hline $\begin{array}{c}\text { Culann } \\
(N W)\end{array}$ & & -19.9 & 161.5 & & & & & & \\
\hline $\mathrm{Y}$ & $03-27-15$ & $-50.0 \pm 1.7$ & $195.5 \pm 2.4$ & 0.61 & & $8.1 \pm 1.5$ & & & \\
\hline
\end{tabular}




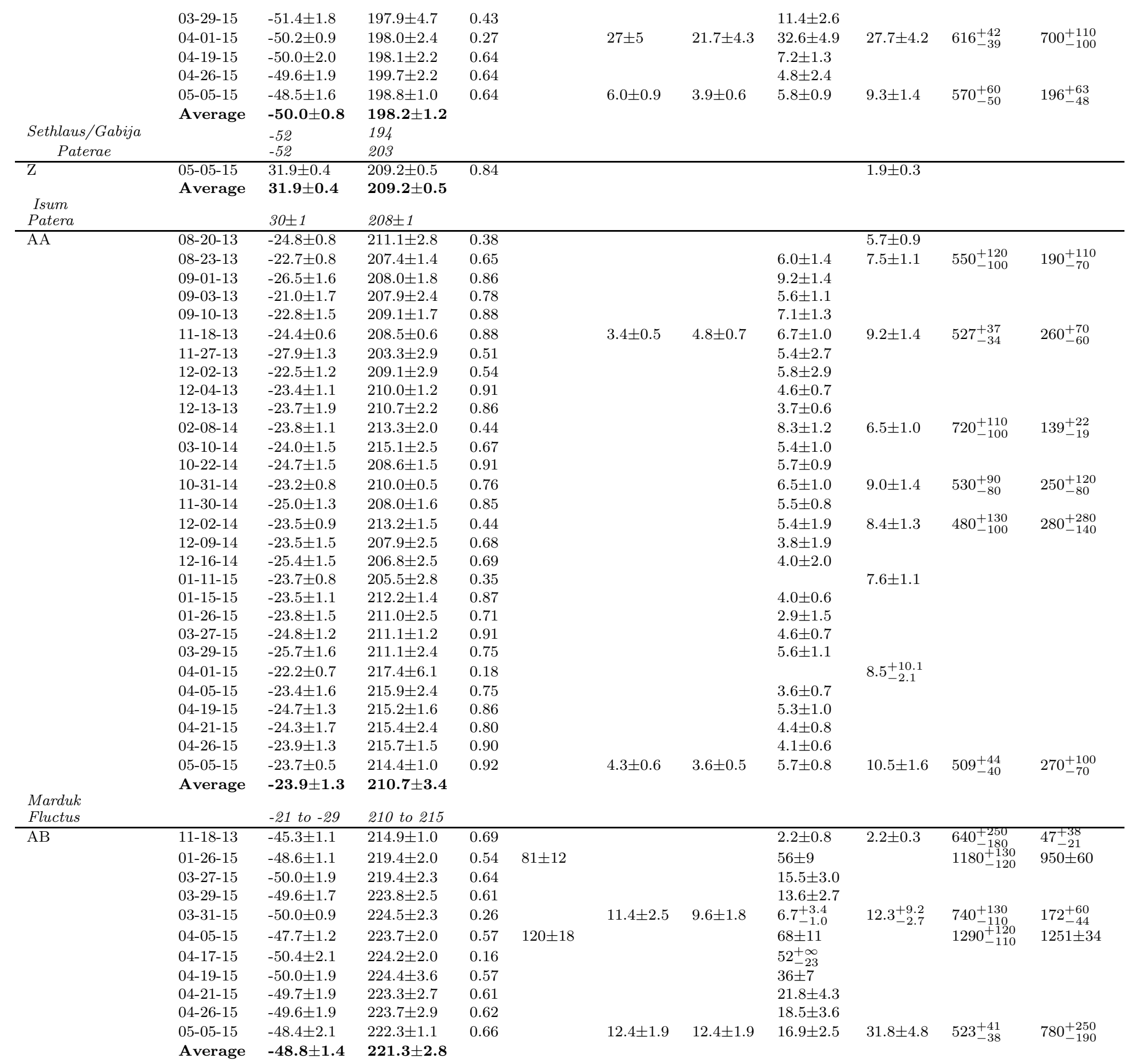




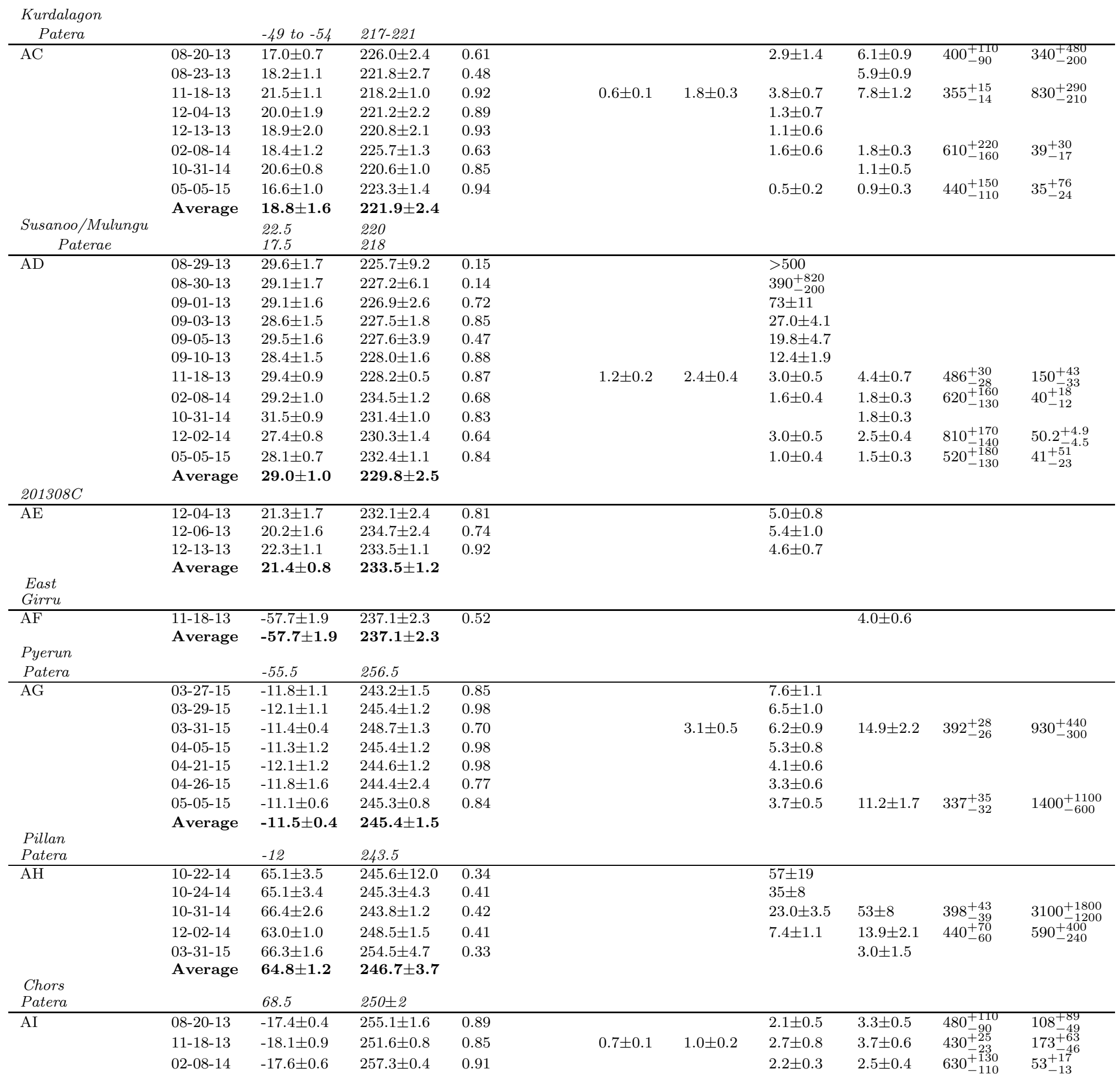




\begin{tabular}{|c|c|c|c|c|c|c|c|c|c|c|c|}
\hline & $10-31-14$ & $-18.1 \pm 0.7$ & $253.1 \pm 0.4$ & 0.94 & & \multirow[b]{2}{*}{$1.1 \pm 0.2$} & \multirow{9}{*}{$1.1 \pm 0.2$} & $2.7 \pm 0.4$ & $3.3 \pm 0.5$ & $580_{-100}^{+120}$ & $78_{-22}^{+30}$ \\
\hline & $12-02-14$ & $-20.3 \pm 1.6$ & $253.6 \pm 0.5$ & 0.87 & & & & $3.4 \pm 0.5$ & $4.6 \pm 0.7$ & $458_{-25}^{+27}$ & $191_{-43}^{+56}$ \\
\hline & $01-26-15$ & $-19.2 \pm 2.0$ & $255.2 \pm 2.1$ & 0.94 & & \multirow{7}{*}{$0.8 \pm 0.1$} & & $0.3 \pm 0.2$ & & & \\
\hline & $03-31-15$ & $-19.4 \pm 0.7$ & $260.2 \pm 2.5$ & 0.80 & & & & $1.1 \pm 0.4$ & $1.7 \pm 0.3$ & $574_{-43}^{+47}$ & $41_{-9}^{+11}$ \\
\hline & $04-19-15$ & $-16.7 \pm 1.2$ & $258.2 \pm 3.6$ & 0.47 & & & & $7.0 \pm 3.5$ & & & \\
\hline & $05-05-15$ & $-19.2 \pm 1.0$ & $257.9 \pm 1.8$ & 0.67 & & & & $1.5 \pm 0.6$ & & & \\
\hline & $11-23-15$ & $-17.3 \pm 0.7$ & $256.7 \pm 2.9$ & 0.31 & & & & & $4.1 \pm 0.7$ & & \\
\hline & Average & $-18.1 \pm 1.0$ & $255.0 \pm 2.5$ & & & & & & & & \\
\hline Pele & & -19 & 256 & & & & & & & & \\
\hline \multirow[t]{5}{*}{$\overline{\mathrm{AJ}}$} & $01-10-15$ & $-59.8 \pm 2.1$ & $264.3 \pm 4.4$ & 0.43 & & & & $55 \pm 12$ & & & \\
\hline & $01-12-15$ & $-57.7 \pm 0.8$ & $269.6 \pm 6.0$ & 0.19 & & & & $13.4_{-3.2}^{+12.6}$ & $20 \pm 8$ & $430_{-140}^{+200}$ & $530_{-250}^{+480}$ \\
\hline & $01-15-15$ & $-57.3 \pm 1.9$ & $263.5 \pm 4.9$ & 0.45 & & & & $8.5 \pm 4.2$ & & & \\
\hline & 03-31-15 & $-58.3 \pm 0.9$ & $266.9 \pm 1.8$ & 0.48 & & & & & $3.1 \pm 0.5$ & & \\
\hline & Average & $-58.1 \pm 0.9$ & $266.2 \pm 2.4$ & & & & & & & & \\
\hline Mithra & & -59 & 266.5 & & & & & & & & \\
\hline \multirow[t]{9}{*}{$\mathrm{AK}$} & $02-08-14$ & $-38.6 \pm 0.3$ & $289.5 \pm 0.4$ & 0.75 & & & \multirow{5}{*}{$12.8 \pm 1.9$} & $15.9 \pm 2.4$ & $16.9 \pm 2.6$ & $570_{-70}^{+80}$ & $430_{-100}^{+140}$ \\
\hline & $02-10-14$ & $-38.3 \pm 0.3$ & $290.6 \pm 0.4$ & 0.71 & & & & $17.6 \pm 2.6$ & $21.3 \pm 3.2$ & $561_{-46}^{+50}$ & $550_{-120}^{+150}$ \\
\hline & $03-12-14$ & $-42.2 \pm 1.9$ & $292.4 \pm 2.2$ & 0.73 & & & & $7.9 \pm 1.5$ & & & \\
\hline & $10-31-14$ & $-37.9 \pm 1.0$ & $286.5 \pm 1.8$ & 0.59 & & & & $4.7 \pm 0.7$ & $3.2 \pm 0.5$ & $850_{-130}^{+160}$ & $69.8_{-3.1}^{+3.2}$ \\
\hline & $12-02-14$ & $-37.0 \pm 0.9$ & $289.1 \pm 1.0$ & 0.77 & & & & & $1.9 \pm 0.3$ & & \\
\hline & $03-31-15$ & $-37.0 \pm 1.0$ & $293.6 \pm 0.8$ & 0.79 & & & \multirow[t]{2}{*}{$1.1 \pm 0.2$} & $1.5 \pm 0.3$ & $1.7 \pm 0.3$ & $590 \pm 60$ & $40_{-9}^{+11}$ \\
\hline & $04-02-15$ & $-38.4 \pm 1.1$ & $290.6 \pm 2.7$ & 0.54 & & \multirow{4}{*}{$4.3 \pm 0.6$} & & & $2.5 \pm 0.5$ & & \\
\hline & $12-25-15$ & $-38.8 \pm 0.5$ & $285.9 \pm 1.6$ & 0.56 & & & $5.3 \pm 0.8$ & $7.1 \pm 1.1$ & $12.1 \pm 1.8$ & $521_{-36}^{+39}$ & $320_{-70}^{+100}$ \\
\hline & Average & $-38.4 \pm 1.5$ & $290.0 \pm 2.4$ & & & & & & & & \\
\hline PV59 & & -41 & 287 & & & & & & & & \\
\hline \multirow[t]{9}{*}{$\overline{\mathrm{AL}}$} & $11-18-13$ & $-56.8 \pm 1.4$ & $279.7 \pm 4.7$ & 0.32 & & & & & $2.7 \pm 1.4$ & & \\
\hline & $12-06-13$ & $-58.0 \pm 2.0$ & $285.7 \pm 3.1$ & 0.52 & & & & $7.1 \pm 3.6$ & & & \\
\hline & $02-10-14$ & $-56.1 \pm 1.0$ & $294.0 \pm 1.5$ & 0.52 & & & $4.0 \pm 0.6$ & & & & \\
\hline & $10-31-14$ & $-56.0 \pm 1.4$ & $281.1 \pm 1.9$ & 0.44 & & & & $5.6 \pm 1.4$ & $5.5 \pm 0.8$ & $630_{-120}^{+150}$ & $119_{-34}^{+47}$ \\
\hline & $12-02-14$ & $-60.0 \pm 1.1$ & $284.1 \pm 1.3$ & 0.50 & & & & $5.4 \pm 1.2$ & $4.3 \pm 0.6$ & $720_{-140}^{+170}$ & $89_{-17}^{+-21}$ \\
\hline & $03-31-15$ & $-57.5 \pm 2.9$ & $295.2 \pm 0.8$ & 0.54 & & $5.3 \pm 0.8$ & $3.6 \pm 0.5$ & $3.2 \pm 1.1$ & $3.3 \pm 0.5$ & $890_{-100}^{+110}$ & $63.1_{-4.3}^{+4.6}$ \\
\hline & $11-23-15$ & $-58.3 \pm 1.7$ & $285.3 \pm 2.5$ & 0.39 & & & & $4.5 \pm 2.3$ & $7.0 \pm 1.1$ & $470_{-110}^{+140}$ & $250_{-130}^{+280}$ \\
\hline & $12-25-15$ & $-56.6 \pm 1.0$ & $290.6 \pm 2.4$ & 0.42 & & & & $3.1 \pm 1.1$ & $4.3 \pm 0.6$ & $510_{-120}^{+160}$ & $120_{-60}^{+130}$ \\
\hline \multirow{2}{*}{\multicolumn{12}{|c|}{$\begin{array}{c}N \text { Lerna } \\
\text { Regio }\end{array}$}} \\
\hline & & & & & & & & & & & \\
\hline \multirow[t]{2}{*}{$\mathrm{AM}$} & $12-25-15$ & $39.4 \pm 0.8$ & $304.3 \pm 1.1$ & 0.71 & & $16.3 \pm 2.4$ & $28.0 \pm 4.2$ & $43 \pm 6$ & $108 \pm 16$ & $412_{-22}^{+23}$ & $5300_{-1400}^{+1900}$ \\
\hline & Average & $\mathbf{3 9 . 4} \pm \mathbf{0 . 8}$ & $304.3 \pm 1.1$ & & & & & & & & \\
\hline $\begin{array}{c}\text { Amaterasu } \\
\text { Patera }\end{array}$ & & $38 \pm 1$ & $306.5 \pm 2$ & & & & & & & & \\
\hline \multirow[t]{15}{*}{$\mathrm{AN}$} & $08-15-13$ & $-40.5 \pm 2.2$ & $305.5 \pm 2.6$ & 0.60 & $500 \pm 80$ & & & $320 \pm 80$ & & $1230_{-130}^{+150}$ & $5550_{-490}^{+530}$ \\
\hline & $08-20-13$ & $-40.1 \pm 1.4$ & $302.1 \pm 1.4$ & 0.70 & $4.0 \pm 1.9$ & & & $23.0 \pm 3.5$ & & $560_{-90}^{+100}$ & $\begin{array}{l}750_{-360}^{+690} \\
\end{array}$ \\
\hline & $08-22-13$ & $-39.3 \pm 1.8$ & $305.4 \pm 1.8$ & 0.74 & & & & $21.0 \pm 3.2$ & $35 \pm 5$ & $460_{-50}^{+60}$ & $\begin{array}{l}1380_{-490}^{+750} \\
\end{array}$ \\
\hline & $09-07-13$ & $-41.0 \pm 1.9$ & $304.8 \pm 2.9$ & 0.66 & & & & $7.7 \pm 1.5$ & & & \\
\hline & $11-29-13$ & $-42.1 \pm 2.1$ & $304.9 \pm 2.1$ & 0.74 & & & & $4.3 \pm 0.6$ & & & \\
\hline & $12-15-13$ & $-39.7 \pm 1.8$ & $303.0 \pm 2.3$ & 0.73 & & & & $2.9 \pm 1.4$ & & & \\
\hline & $02-10-14$ & $-37.8 \pm 0.4$ & $307.9 \pm 1.1$ & 0.78 & & & $3.5 \pm 0.5$ & $3.3 \pm 0.5$ & $2.6 \pm 0.4$ & $860_{-100}^{+110}$ & $54.1_{-2.9}^{+3.1}$ \\
\hline & $03-10-14$ & $-41.9 \pm 1.6$ & $300.2 \pm 3.8$ & 0.55 & & & & $5.2 \pm 2.6$ & & & \\
\hline & $03-12-14$ & $-39.0 \pm 1.9$ & $309.9 \pm 2.2$ & 0.77 & & & & $2.7 \pm 1.4$ & & & \\
\hline & $03-28-14$ & $-37.2 \pm 1.6$ & $306.0 \pm 2.5$ & 0.68 & & & & $4.9 \pm 1.0$ & & & \\
\hline & $01-12-15$ & $-33.7 \pm 1.4$ & $304.9 \pm 1.5$ & 0.69 & & & & $1.9 \pm 0.3$ & $2.0 \pm 0.3$ & $690_{-120}^{+150}$ & $40_{-8}^{+10}$ \\
\hline & $03-31-15$ & $-37.4 \pm 1.1$ & $307.2 \pm 1.0$ & 0.76 & & $2.8 \pm 0.4$ & $1.9 \pm 0.3$ & $1.6 \pm 0.4$ & $1.0 \pm 0.4$ & $940 \pm 60$ & $29.3 \pm 0.8$ \\
\hline & $04-02-15$ & $-39.0 \pm 2.9$ & $308.3 \pm 1.2$ & 0.66 & & & & $2.2 \pm 0.8$ & $1.3 \pm 0.5$ & $680_{-200}^{+290}$ & $29_{-10}^{+15}$ \\
\hline & 04-09-15 & $-39.1 \pm 1.9$ & $309.0 \pm 2.2$ & 0.76 & & & & $5.5 \pm 0.8$ & & & \\
\hline & Average & $-38.7 \pm 2.0$ & $306.0 \pm 2.6$ & & & & & & & & \\
\hline
\end{tabular}




\begin{tabular}{|c|c|c|c|c|c|c|c|c|c|c|c|}
\hline Patera & & -42 & 304 & & & & & & & & \\
\hline \multirow[t]{8}{*}{$\mathrm{AO}$} & $08-15-13$ & $-55.2 \pm 2.1$ & $307.5 \pm 2.1$ & 0.47 & $92 \pm 15$ & & & $270 \pm 70$ & & $720_{-90}^{+100}$ & $4800_{-1800}^{+2800}$ \\
\hline & $08-20-13$ & $-53.0 \pm 2.1$ & $304.0 \pm 3.0$ & 0.54 & $4.9 \pm 2.4$ & & & $40 \pm 6$ & & $530_{-70}^{+90}$ & $1500_{-700}^{+1800}$ \\
\hline & $08-22-13$ & $-55.6 \pm 1.7$ & $309.5 \pm 1.7$ & 0.53 & $9.3 \pm 2.6$ & & & $50 \pm 8$ & $69 \pm 11$ & $577_{-40}^{+43}$ & $\begin{array}{c}1580_{-300}^{+370} \\
\end{array}$ \\
\hline & $08-29-13$ & $-55.7 \pm 2.5$ & $309.2 \pm 3.0$ & 0.56 & & & & $31 \pm 6$ & & & \\
\hline & $09-05-13$ & $-55.0 \pm 2.5$ & $307.1 \pm 4.6$ & 0.53 & & & & $14.6 \pm 3.1$ & & & \\
\hline & $09-07-13$ & $-56.2 \pm 2.5$ & $307.5 \pm 4.3$ & 0.49 & & & & $17.1 \pm 3.8$ & & & \\
\hline & $04-02-15$ & $-57.9 \pm 0.8$ & $305.0 \pm 1.9$ & 0.44 & & & & & $2.8 \pm 0.4$ & & \\
\hline & Average & $-56.0 \pm 1.3$ & $307.2 \pm 1.8$ & & & & & & & & \\
\hline \multicolumn{12}{|l|}{$\begin{array}{c}\text { Heno } \\
\text { Patera }\end{array}$} \\
\hline \multirow[t]{44}{*}{$\overline{\mathrm{AP}}$} & $08-15-13$ & $12.0 \pm 1.4$ & $309.0 \pm 1.4$ & 0.87 & $7.7 \pm 1.4$ & & & $60 \pm 10$ & & $550_{-31}^{+33}$ & $2000_{-500}^{+700}$ \\
\hline & $08-20-13$ & $11.9 \pm 0.8$ & $308.7 \pm 1.2$ & 0.83 & $13.0 \pm 3.3$ & & & $133 \pm 27$ & $200 \pm 33$ & $514_{-27}^{+29}$ & $5800_{-1100}^{+1400}$ \\
\hline & $08-22-13$ & $11.7 \pm 1.2$ & $308.4 \pm 1.0$ & 0.97 & $7.5 \pm 1.3$ & & & $136 \pm 20$ & $205 \pm 34$ & $\begin{array}{l}-27 \\
470 \pm 16\end{array}$ & $7600_{-1300}^{+1100}$ \\
\hline & $08-29-13$ & $11.3 \pm 1.4$ & $307.1 \pm 1.4$ & 0.98 & & & & $126 \pm 19$ & & & \\
\hline & $09-03-13$ & $10.9 \pm 1.5$ & $308.4 \pm 4.7$ & 0.38 & & & & $50 \pm 14$ & & & \\
\hline & 09-05-13 & $11.3 \pm 1.4$ & $307.4 \pm 1.6$ & 0.91 & & & & $73 \pm 11$ & & & \\
\hline & $09-07-13$ & $12.0 \pm 1.4$ & $307.0 \pm 1.7$ & 0.87 & & & & $84 \pm 13$ & & & \\
\hline & $09-09-13$ & $10.2 \pm 1.5$ & $310.3 \pm 5.0$ & 0.27 & & & & $37 \pm 19$ & & & \\
\hline & $09-10-13$ & $12.0 \pm 1.4$ & $295.1 \pm 5.3$ & 0.35 & & & & $14.8 \pm 4.6$ & & & \\
\hline & $11-18-13$ & $14.2 \pm 0.5$ & $309.5 \pm 3.7$ & 0.11 & & & & & $28_{-10}^{+\infty}$ & & \\
\hline & $11-27-13$ & $11.9 \pm 1.4$ & $307.0 \pm 2.6$ & 0.64 & & & & $12.0 \pm 1.8$ & & & \\
\hline & $11-29-13$ & $12.4 \pm 1.1$ & $306.5 \pm 1.1$ & 0.98 & & & & $12.6 \pm 1.9$ & & & \\
\hline & $12-06-13$ & $12.0 \pm 1.1$ & $307.5 \pm 1.5$ & 0.81 & & & & $10.5 \pm 1.6$ & & & \\
\hline & $12-15-13$ & $12.5 \pm 1.0$ & $307.3 \pm 1.1$ & 0.95 & & & & $11.2 \pm 1.7$ & & & \\
\hline & $01-20-14$ & $12.2 \pm 0.6$ & $312.6 \pm 2.9$ & 0.25 & & & & & $17.4 \pm 3.8$ & & \\
\hline & $02-08-14$ & $12.6 \pm 0.9$ & $310.7 \pm 0.3$ & 0.78 & & & & $8.5 \pm 1.3$ & $24.7 \pm 3.8$ & $353_{-33}^{+36}$ & $2400_{-1000}^{+1700}$ \\
\hline & $02-10-14$ & $12.6 \pm 0.3$ & $310.0 \pm 0.3$ & 0.97 & & & $2.1 \pm 0.3$ & $11.1 \pm 1.7$ & $31.8 \pm 4.8$ & $318_{-44}^{+51}$ & $4700_{-2300}^{+4600}$ \\
\hline & $03-10-14$ & $12.1 \pm 1.2$ & $314.0 \pm 2.7$ & 0.54 & & & & $6.4 \pm 3.2$ & & & \\
\hline & $03-12-14$ & $11.9 \pm 1.2$ & $311.3 \pm 1.2$ & 0.97 & & & & $7.7 \pm 1.1$ & & & \\
\hline & $03-28-14$ & $13.1 \pm 1.3$ & $309.5 \pm 1.5$ & 0.86 & & & & $7.6 \pm 1.1$ & & & \\
\hline & $10-03-14$ & $12.2 \pm 1.4$ & $306.9 \pm 1.4$ & 0.98 & & & & $127 \pm 19$ & & & \\
\hline & $10-10-14$ & $11.6 \pm 1.4$ & $306.6 \pm 1.6$ & 0.94 & & & & $128 \pm 19$ & & & \\
\hline & $10-24-14$ & $10.8 \pm 1.4$ & $304.7 \pm 2.3$ & 0.68 & & & & $66 \pm 10$ & & & \\
\hline & $10-30-14$ & $11.1 \pm 0.5$ & $309.9 \pm 4.8$ & 0.07 & & & & $40_{-28}^{+\infty}$ & $43_{-25}^{+\infty}$ & & \\
\hline & $10-31-14$ & $12.2 \pm 0.5$ & $306.3 \pm 1.7$ & 0.45 & & & & $78 \pm 12$ & $\begin{array}{c}-25 \\
155 \pm 23\end{array}$ & $416_{-25}^{+27}$ & $7900_{-1900}^{+2500}$ \\
\hline & $11-25-14$ & $10.6 \pm 1.2$ & $307.0 \pm 1.5$ & 0.90 & & & & $55 \pm 8$ & & & \\
\hline & $11-27-14$ & $11.1 \pm 1.2$ & $305.6 \pm 1.5$ & 0.86 & & & & $54 \pm 8$ & & & \\
\hline & $11-29-14$ & $9.4 \pm 1.2$ & $298.9 \pm 7.8$ & 0.12 & & & & $59_{-31}^{+\infty}$ & & & \\
\hline & $11-30-14$ & $9.8 \pm 1.2$ & $293.1 \pm 3.7$ & 0.41 & & & & $10.5 \pm 2.5$ & & & \\
\hline & $12-02-14$ & $10.2 \pm 1.1$ & $305.3 \pm 0.6$ & 0.85 & & $12.3 \pm 1.8$ & & $47 \pm 7$ & $122 \pm 18$ & $380 \pm 13$ & $8100_{-1600}^{+2000}$ \\
\hline & $12-06-14$ & $11.7 \pm 1.2$ & $306.0 \pm 1.4$ & 0.90 & & & & $29.0 \pm 4.3$ & & & \\
\hline & $12-09-14$ & $10.7 \pm 1.3$ & $303.3 \pm 2.6$ & 0.58 & & & & $24.1 \pm 3.8$ & & & \\
\hline & $12-16-14$ & $11.2 \pm 1.2$ & $305.1 \pm 2.7$ & 0.52 & & & & $14.0 \pm 2.9$ & & & \\
\hline & $12-18-14$ & $12.0 \pm 1.2$ & $306.5 \pm 1.3$ & 0.95 & & & & $24.0 \pm 3.6$ & & & \\
\hline & $01-10-15$ & $12.9 \pm 1.1$ & $307.2 \pm 1.2$ & 0.96 & & & & $12.4 \pm 1.9$ & & & \\
\hline & $01-12-15$ & $11.2 \pm 0.6$ & $306.3 \pm 0.4$ & 0.82 & & & $7.7 \pm 1.2$ & $23.2 \pm 3.5$ & $66 \pm 10$ & $352 \pm 12$ & $6100_{-1300}^{+1600}$ \\
\hline & $01-14-15$ & $12.4 \pm 1.1$ & $307.4 \pm 1.2$ & 0.92 & & & & $18.7 \pm 2.8$ & & & \\
\hline & $01-26-15$ & $11.6 \pm 1.1$ & $307.6 \pm 2.7$ & 0.52 & & & & $7.7 \pm 3.9$ & & & \\
\hline & $03-29-15$ & $12.7 \pm 1.2$ & $311.7 \pm 4.2$ & 0.37 & & & & $11 \pm 5$ & & & \\
\hline & $03-31-15$ & $12.3 \pm 0.4$ & $312.0 \pm 0.8$ & 0.92 & & & $2.4 \pm 0.4$ & $9.8 \pm 1.5$ & $29.8 \pm 4.5$ & $322 \pm 9$ & $4400_{-900}^{+1100}$ \\
\hline & $04-02-15$ & $12.7 \pm 1.0$ & $311.5 \pm 0.8$ & 0.86 & & & & $9.3 \pm 1.4$ & $28.3 \pm 4.2$ & $350_{-30}^{+32}$ & $2800_{-1100}^{+1800}$ \\
\hline & $04-04-15$ & $12.7 \pm 0.7$ & $318.9 \pm 1.8$ & 0.42 & & & & & $15.4 \pm 2.3$ & & \\
\hline & $04-05-15$ & $12.0 \pm 1.2$ & $307.0 \pm 2.7$ & 0.54 & & & & $6.0 \pm 3.0$ & & & \\
\hline & 04-09-15 & $12.4 \pm 1.2$ & $311.7 \pm 1.2$ & 0.96 & & & & $8.7 \pm 1.3$ & & & \\
\hline
\end{tabular}




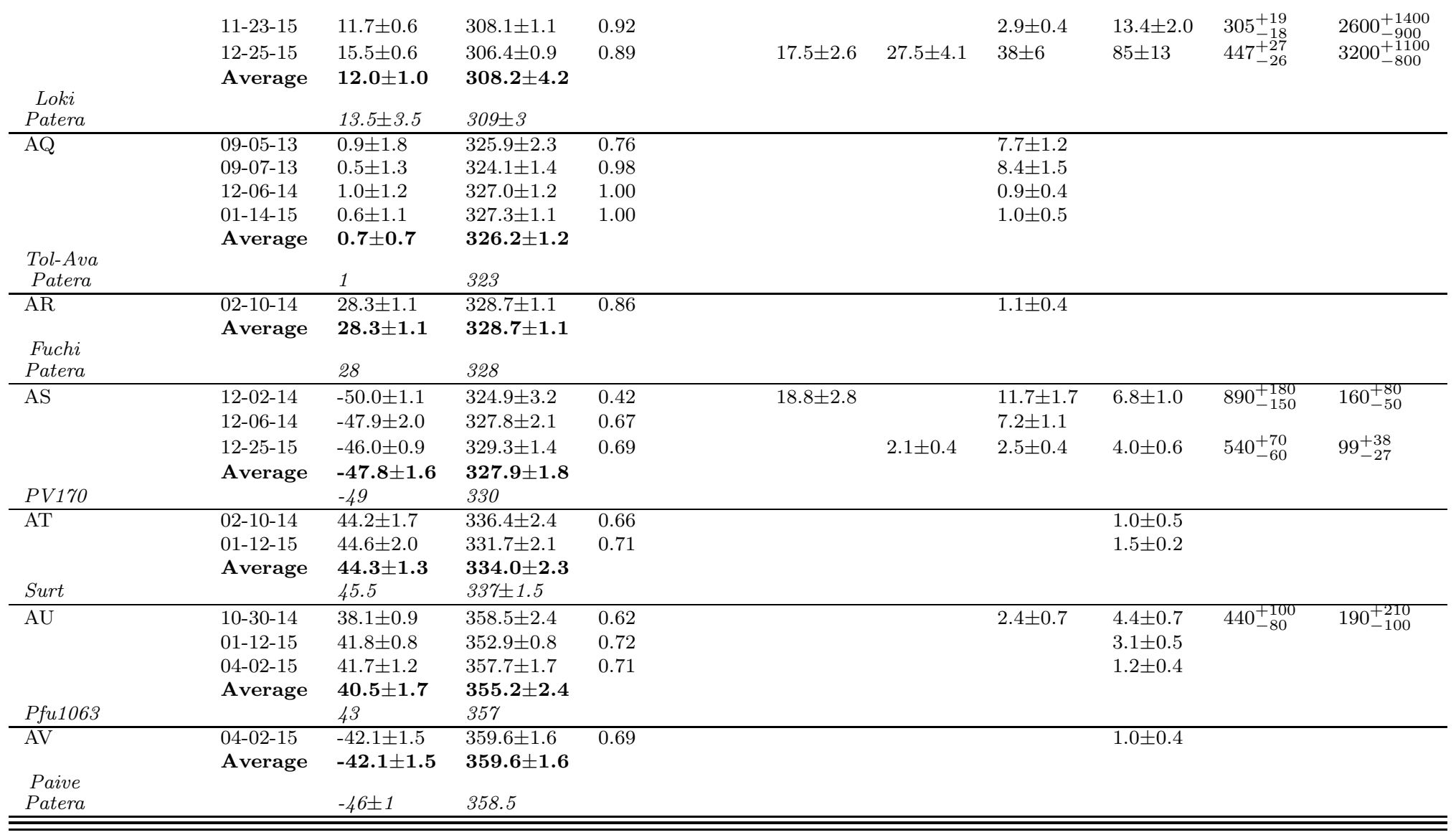

\section{NOTES:}

(1) Data from 2013 Aug 15, 20, and 22 were previously published in de Pater et al. (2014a), and data from 2013 Aug 29 through Sep were previously published in de Kleer et al. (2014). Observations of Pillan Patera have been previously published in de Pater et al. (2016a). Flux densities and temperatures re-derived in this work are consistent with the published values.

(2) Uncertainties for close pairs of hot spots may be higher than quoted if emission arises from both sources. These include Kanehekili Fluctus/Janus Patera, and Sigurd/Itzamna Paterae.

(3) The hot spot at Rarog Patera may cover two unresolved hot spots; the locations are consistent with Sengen and Rarog Paterae.

(4) The locations of the hot spots identified as Susanoo/Mulungu Paterae and Gabija/Sethlaus Patarae are consistent with either source.

(5) On 03-31-15 and 04-01-15, the detected emission attributed to Kurdalagon Patera and Gabija/Sethlaus Patarae may include a component from the other of these two sources.

(6) Locations of surface features from Williams et al. (2011a; 2011b) 
References

Blaney, D.L., Johnson, T.V., Matson, D.L., Veeder, G.J., 1995. Volcanic eruptions on Io: heat flow, resurfacing, and lava composition. Icarus 113, 200-225.

Cruikshank, D.P., Murphy, R.E., 1973. The post-eclipse brightening of Io. Icarus 20, 7-17.

Davies, A.G., Keszthelyi, L.Pl., Williams, D.A., et al., 2001. Thermal signature, eruption style, and eruption evolution at Pele and Pillan on Io. JGeoRes 106, 33079-33103.

Davies, A.G. Volcanism on Io: a Comparison with Earth, Cam. Univ. Press, 2007.

Davies, A.G., Keszthelyi, L.P., Harris, A.J.L., 2010. The thermal signature of volcanic eruptions on Io and Earth. J. Volc. Geo. Res. 194, 75-99.

Davies, A.G., Veeder, G.J., Matson, D.L., and Johnson, T.V., 2012. Charting thermal emission variability at Pele, Janus Patera, and Kanehekili Fluctus with the Galileo NIMS Io Thermal Emission Database (NITED). Icarus $221,466-470$.

Davies, A.G., et al., 2014. Charting thermal emission variability at Amirani with the Galileo NIMS Io Thermal Emission Database (NITED). Icarus 190-199.

Davies, A.G., Veeder, G.J., Matson, D.L., Johnson, T.V., 2015. Map of Io's volcanic heat flow. Icarus 262, 67-78

de Kleer, K., de Pater, I., Davies, A.G., Ádámkovics, M., 2014. Near-infrared monitoring of Io and detection of a violent outburst on 29 August 2013. Icarus 242, 352-364.

de Kleer, K. and de Pater, I., 2016a. Spatial distribution of Io's volcanic activity from near-IR adaptive optics observations on 100 nights in 2013-2015. Icarus, submitted

de Kleer, K. and de Pater, I., 2016b. Io's Loki Patera: Three brightening events in 2013-2015 and a refined magma sea model. in prep

de Pater, I., Marchis, F., Macintosh, B.A., Roe, H.G., Le Mignant, D.L., Graham, J.R., Davies, A.G., 2004. Keck AO observations of Io in and out of eclipse. Icarus 169, 250-263. 
de Pater, I., Gibbard, S., Hammel, H.B., 2006. Evolution of the dusty rings of Uranus. Icarus 180, 186-200.

de Pater, I., Davies, A.G., Ádámkovics, M., Ciardi, D.R., 2014a. Two new, rare, high-effusion outburst eruptions at Rarog and Heno Paterae on Io. Icarus 242, 365-378.

de Pater, I., Davies, A.G., McGregor, A., et al., 2014b. Global near-IR maps from Gemini-N and Keck in 2010, with a special focus on Janus Patera and Kanehekili Fluctus. Icarus 242, 379-395.

de Pater, I., Laver, C., Davies, A.G., et al., 2016a. Io: Eruptions at Pillan, and the time evolution of Pele from 1996 to 2015. Icarus 264, 198-212.

de Pater, I., Davies, A.G., Marchis, F., 2016b. Keck observations of eruptions on Io in 2003-2005. Icarus, accepted. de Pater, I., de Kleer, K., Davies, A., Ádámkovics, M, 2016c. Three decades of Loki Patera observations, in prep Foreman-Mackey , D., Hogg, D.W., Lang, D., et al., 2013. emcee: The MCMC Hammer. PASP 125, 306-312. Geissler, P., McEwen, A., Phillips, C., et al., 2004. Surface changes on Io during the Galileo mission. Icarus 169, 29-64.

Geissler, P. and Goldstein, D., 2004. Plumes and their deposits. In Io After Galileo, ed. R. Lopes and J. Spencer. Geissler, P.E. and McMillan, M.T., 2008. Galileo observations of volcanic plumes on Io. Icarus 197, 505-518.

Gerlach, T.M. and McGee, K.A., 1994. Total sulfur dioxide emissions and pre-eruption vapor-saturated magma at Mount St. Helens, 1980-88. Geo Res Lett. 21, 2833-2836.

Gibbard, S.G., de Pater, I., \& Hammel, H.B., 2005. Near-infrared adaptive optics imaging of the satellites and individual rings of Uranus. Icarus 174, 253.

Hill, D.P. et al., 1993. Seismicity remotely triggered by the magnitude 7.3 Landers, California, earthquake. Science 260, 1617-1623.

Hodapp, K.W., et al., 2003. The Gemini Near-Infrared Imager (NIRI). PASP 115, 1388.

Howell, R.R., Spencer, J.R., Goguen, J.D., et al., 2001. Ground-based observations of volcanism on Io in 1999 and early 2000. J Geo Res 106, 33129-33140. 
Johnson, T.V., Matson, D.L., Blaney, D.L., et al., 1995. Stealth plumes on Io. Geo Res Lett 22, 3293-3296

Keszthelyi L., et al., 2001. Imaging of volcanic activity on Jupiter's moon Io by Galileo during the Galileo Europa Mission and the Galileo Millennium Mission. J. Geo. Res. 106, 33025-33052.

Laver, C., de Pater, I., Marchis, F., 2007. Tvashtar awakening detected in April 2006 with OSIRIS at the W.M. Keck Observatory. Icarus 191, 749-754.

Linde, A.T., Sacks, I.S., 1998. Triggering of volcanic eruptions. Nature 395, 888-890.

Leggett, S.K., Hawarden, T.G., Currie, M.J., et al., 2003. L' and M'standard stars for the Mauna Kea Observatories near-infrared system. MNRAS 345, 144-152.

Legget, S.K., Currie, M.J., Varricatt, W.P., et al., 2006. JHK observations of faint standard stars in the Mauna Kea Observatories near-infrared photometric system. MNRAS 373, 781-792.

Lellouch, E., Belton, M., de Pater, I., et al. 1990. Io's atmosphere from microwave detection of $\mathrm{SO}_{2}$. Nature 346 , $639-641$

Lopes, R.M.C., Kamp, L.W., Smythe, W.D., et al., 2004. Lava lakes on Io: observations of Io's volcanic activity from Galileo NIMS during the 2001 fly-bys. Icarus 169, 140-174.

Lopes, R.M.C., Spencer, J.R., 2007. Io After Galileo. Springer-Praxis publishing, Chichester, UK.

Lopes-Gautier, R., McEwen, A.S., Smythe, W.B., Geissler, P.E., Kamp, L., Davies, A.G., 1999. Active volcanism on Io: Global distribution and variations in activity. Icarus 140, 243-264.

Manga, M., Brodsky, E., 2006. Seismic triggering of eruptions in the far field: Volcanoes and geysers. Ann Rev Earth Planet Sci 34, 263-91.

Marchis, F., Prangé, R., Christou, J., 2000. Adaptive optics mapping of Io's volcanism in the thermal IR (3.8 $\mu \mathrm{m})$. Icarus 148, 384 .

Marchis, F., de Pater, I., Davies, A.G., et al., 2002. High-resolution Keck adaptive optics imaging of violent volcanic activity on Io. Icarus 160,124-131. 
Marchis, M., et al., Keck AO survey of Io global volcanic activity between 2 and 5 microns. Icarus 176, 96-122.

Matson, D., Ransford, G.A., Johnson, T.V., 1981. Heat flow from Io. JGR 86, 1664-1672.

Matson, D.L., Blaney, D,L., Johnson, T.V., et al., 1998. Io and the early Earth, 29th LPSC, Houston, Texas, no. 1650.

Matson, D.L., Davies, A.G., Veeder, G.J, Rathbun, J.A., Johnson, T.V., \& Castillo, J.C., 2006. Io: Loki Patera as a magma sea. JGeoRes 111, E09002.

McEwen. A.S. and Soderblom, L.A., 1983. Two classes of volcanic plumes on Io. Icarus 55, 191-217.

McEwen, A.S., Keszthelyi, L., Spencer, J.R., et al., 1998. High-temperature silicate volcanism on Jupiter's moon Io. Science 281, 87-90.

Milazzo, M.P., et al., 2005. Volcanic activity at Tvashtar Catena, Io. Icarus 179, 235-251.

Murakami, G., Kazui, Y., Atsushi, Y., et al., 2012. EXCEED: an extreme ultraviolet spectrometer onboard SPRINT-A. SpIE 8443, p.2.

Peale, S., Cassen, P., Reynolds, R., 1979. Melting of Io by tidal dissipation. Science, 203, 892-894. Rathbun 2006 Loki

Radebaugh, J., 2005. Formation and Evolution of Paterae on Jupiter's Moon Io. Thesis, Univ. Arizona, Tucson. Rathbun, J.A., and Spencer, J.R., 2010. Ground-based observations of time variability in multiple active volcanoes on Io. Icarus 209, 625-630.

Rathbun, J., Spencer, J.R., Lopes, R.M., et al., 2014. Io's active volcanoes during the New Horizons era: Insights from New Horizons imaging. Icarus 231, 261-272.

Rhoden, A.R., Hurford, T.A., Lorenz, R., Retherford, K., 2015. Linking Europa's plume activity to tides, tectonics, and liquid water. Icarus 253, 169-178.

Scargle, J.D., 1982. Studies in astronomical time series analysis. II - Statistical aspects of spectral analysis of unevenly spaced data. ApJ 263, 835-853. 
Spencer, J.R., Schneider, N.M., 1996. Io in the even of the Galileo mission. AREPS 24, 125-190.

Schneider, N.M., and Baganel, R., 2004. Io's neutral clouds, plasma torus, and magnetospheric interaction. In Io After Galileo, Springer Publishing.

Spencer, J.R., Stern, S.A., Cheng, A.F. et al., 2007. Io volcanism seen by New Horizons: A major eruption of the Tvashtar volcano. Science 318, 240-243.

Spencer, J.R., Stansberry, J.A., Dumas, C., et al., 1997. A history of high-temperature volcanism: February 1995 to May 1997. GRL 24, 2451-2454.

Spencer, J.R., Lellouch, E., Richter, M.J., et al., 2005. Mid-infrared detection of large longitudinal asymmetries in Io's $\mathrm{SO}_{2}$ atmosphere. Icarus 176, 283-304.

Stansberry, J.A., Spencer, J.R., Howell, R.R., et al., 1997. Violent silicate volcanism on Io in 1996. Geo Res Lett, 24 , p. 2455.

Strom, R.G. and Schneider, N.M., 1981. Volcanic eruption plumes on Io. JGR 86, 8593-8620.

Sutton, A.J. and Elias, T., 2014. One Hundred Volatile Years of Volcanic Gas Studies at the Hawaiian Volcano Observatory. In Characteristics of Hawaiian Volcanoes, USGS Professional Paper 1801.

Tsang, C.C., Rathbun, J.A., Spencer, J.R., et al., 2014. Io's hot spots in the near-infrared detected by LEISA during the New Horizons flyby. J Geo Res, 119, 2222-2238.

Tsuchiya, F., Yoshioka, K., Kimura, T., Murakami, G., et al., 2015. Io's volcanic influence on the Io plasma torus: HISAKI observation in 2015. Fall AGU Meeting, SM31B-2489.

Ueno, M., Yoshikawa, I., Yamazaki, A., et al., 2011. SPRINT-A/EXCEED mission. EPSC-DPS, p. 811.

Veeder, G.J., Matson, D.L., Johnson, T.V., Blaney, D.L., and Goguen, J.D., 1994. Io's heat flow from infrared radiometry: 1983-1993. JGR vol. 99, no. E8, pp. 17095-17162.

Veeder, G.J., et al., 2011. Io: Heat flow from dark paterae. Icarus 212, 236-261. 
Veeder, G.J., Davies, A.G., Matson, D.L., Johnson, T.V., Williams, D.A. and Radebaugh, J., 2012. Io: Volcanic thermal sources and global heat flow. Icarus 219, 701-722.

Veeder, G.J., et al., 2015. Io: Heat flow from small volcanic features. Icarus 245, 379-410.

Williams, S.N. 1995. Erupting neighbors - at last. Science 267, 340-341.

Williams, D.A. et al., 2011a. Geologic map of Io, USGS Scientific Investigations Map 3168, scale 1:15,000,000.

Williams, D.A., Keszthelyi, L.P., Crown, D.A., 2011b. Volcanism on Io: New insights from global geologic mapping. Icarus 214, 91-112.

Yoneda, M., Kagitani, M., Tsuchiya, F., et al., 2015. Brightening event seen in observations of Jupiter's extended sodium nebula. Icarus 261, 31-33.

Zechmeister, M. and Kürster, M., 2009. The generalized Lomb-Scargle periodogram. A\&A 496, 577-584. 